Tulio Anselmo dos Santos Valentim

Relação VQ na Avaliação da Estabilidade

de Tensão

Dissertação de Mestrado

Dissertação apresentada como requisito parcial para obtenção do grau de Mestre pelo Programa de Pósgraduação em Engenharia Elétrica do Departamento de Engenharia Elétrica do da PUC-Rio.

Orientador: Prof. Ricardo Bernardo Prada

Rio de Janeiro

Março de 2020 
Tulio Anselmo dos Santos Valentim

\title{
Relação VQ na Avaliação da Estabilidade de Tensão
}

\begin{abstract}
Dissertação apresentada como requisito parcial para obtenção do grau de Mestre pelo Programa de Pósgraduação em Engenharia Elétrica do Departamento de Engenharia Elétricado da PUC-Rio. Aprovada pela Comissão Examinadora abaixo:
\end{abstract}

Prof. Ricardo Bernardo Prada

Orientador

Departamento de Engenharia Elétrica - PUC-Rio

Prof. Marcos Vinicius Pimentel Teixeira Instituto Militar de Engenharia - IME

Prof. Delberis Araujo Lima

Departamento de Engenharia Elétrica - PUC-Rio

Rio de Janeiro, 10 de Março de 2020 
Todos os direitos reservados. A reprodução, total ou parcial do trabalho, é proibida sem a autorização da universidade, do autor e do orientador.

\section{Tulio Anselmo dos Santos Valentim}

Graduou-se em Engenharia Eletrônica e de Computação pela Universidade Federal do Rio de Janeiro em 2009. Trabalho como engenheiro eletrônico nas áreas de planejamento energético e implantação de projetos de geração em Furnas. Atualmente atua como consultor na área de certificação de projetos eólicos e solares da UL Renewable.

Ficha Catalográfica

Anselmo dos Santos Valentim, Tulio

Relação VQ na Avaliação da Estabilidade de Tensão. Tulio Anselmo dos Santos Valentim; orientador: Ricardo Bernardo Prada. - 2020.

v., 100 f: il. color. ; $30 \mathrm{~cm}$

Dissertação (mestrado) - Pontifícia Universidade Católica do Rio de Janeiro, Departamento de Engenharia Elétrica, 2020.

Inclui bibliografia

1. Engenharia Elétrica - Teses. 2. Sistemas de Potência - Teses. 3. Estabilidade de tensão;. 4. Margem de potência reativa;. 5. Curva QV;. 6. Matriz D'. I. Bernardo Prada, Ricardo. II. Pontifícia Universidade Católica do Rio de Janeiro . Departamento de Engenharia Elétrica. III. Título. 
A Lívia, minha companheira e amiga, pelo apoio emocional e suporte técnico. 


\section{Agradecimentos}

A Deus por mostrar-me o caminho a seguir, por demonstrar-me mais uma vez que todas as coisas são possíveis se temos fé.

A meu orientador, Ricardo Bernardo Prada, pelo permanente apoio nas diferentes etapas de desenvolvimento nesta dissertação, por sua atenção e paciência.

A minha família, pais e irmãos, que sempre me apoiaram em meus desafios e sonhos.

Ao povo brasileiro por terem dado suporte financeiro nos meus estudos. A todos os amigos do curso de Pós-Graduação em Engenharia Elétrica, muito especialmente a Vanessa Atencia, Oscar Cuaresma, Marília Zacarias, Diego Cardoso e André Milhorance.

Por último, mas não menos importante, gostaria de agradecer à CAPES pelo apoio financeiro e à PUC-Rio pela bolsa de isenção de mensalidades do doutorado.

O presente trabalho foi realizado com apoio da Coordenação de Aperfeiçoamento de Pessoal de Nível Superior - Brasil (CAPES) - Código de Financiamento 001. 


\section{Resumo}

Anselmo dos Santos Valentim, Tulio; Prada, Ricardo Bernardo (Orientador). Relação VQ na Avaliação da Estabilidade de Tensão. Rio de Janeiro, 2020. 100p. Dissertação de Mestrado - Departamento de Engenharia Elétrica, Pontifícia Universidade Católica do Rio de Janeiro .

O fenômeno de estabilidade de tensão é um problema associado ao fluxo de potência ativa e reativa nas linhas de transmissão. A manifestação mais conhecida do fenômeno é a existência de uma máxima carga que pode ser alimentada pela rede. Outra possibilidade é a existência de uma máxima injeção de potência ativa e reativa fornecida por geradores e compensadores. A curva QV possibilita o cálculo da margem $\mathrm{Q}$, que corresponde à distância entre o caso-base e ponto de fronteira da curva. A margem Q, supostamente, é um índice para avaliação da estabilidade do sistema, determinando uma operação normal quando a inclinação da curva é positiva. $O$ método da matriz $D^{\prime}$ também fornece a relação $Q$ e $V$ de cada barra, avaliando, supostamente, as condições de estabilidade do sistema. Nesta dissertação foi proposta a análise desses dois índices, com o objetivo de explicitar as premissas, similaridades e diferenças das duas maneiras de se calcular a relação VQ. E assim, avaliar e identificar possíveis resultados não coerentes.

\section{Palavras-chave}

Estabilidade de tensão; Margem de potência reativa; Curva QV; Matriz D'. 


\section{Abstract}

Anselmo dos Santos Valentim, Tulio; Bernardo Prada, Ricardo (Advisor). Relation $\mathrm{VQ}$ in the Evaluation of Voltage Stability. Rio de Janeiro, 2020. 100p. Dissertação de Mestrado - Departamento de Engenharia Elétrica, Pontifícia Universidade Católica do Rio de Janeiro.

The phenomenon of voltage stability is a problem associated with active and reactive power flow in transmission lines. The best-known manifestation of the event is the existence of a maximum load that can be supplied by the grid. Another possibility is the existence of a maximum injection of active and reactive power provided by generators and compensators. The QV curve enables the calculation of the $\mathrm{Q}$ margin, which corresponds to the distance between the base case and the curve's inflection point. The $\mathrm{Q}$ margin, supposedly, is an index for evaluating the system stability, consisting of normal operation when the slope of the curve is positive. The matrix method D' also provides the relation $\mathrm{Q}$ and $\mathrm{V}$ of each bar evaluating, supposedly, conditions of system stability. In this dissertation, an analysis of these two indices was proposed to clarify the assumptions, similarities, and differences between the two ways of calculating the VQ relation. And so, evaluate and identify possible non-coherent results.

\section{Keywords}

Voltage Stability; Reactive power margin; QV curve; Matrix D'. 


\section{Sumário}

1 Introdução $\quad 14$

1.1 Considerações Gerais 14

$\begin{array}{lll}1.2 & \text { Objetivos } & 14\end{array}$

$\begin{array}{lll}1.3 & \text { Estrutura do Trabalho } & 15\end{array}$

2 Fluxo de Potência $\quad 16$

$\begin{array}{lll}2.1 & \text { Introdução } & 16\end{array}$

$\begin{array}{ll}2.2 & \text { Equações de Carga } \\ & 17\end{array}$

2.3 Desenvolvendo a Matriz de Admitância 19

2.4 Método de Newton-Raphson para Solução do Fluxo de Potência 22

2.5 Sumário do Capítulo 26

3 Estabilidade de Tensão $\quad 27$

3.1 Límite de Estabilidade Estática Angular 27

3.2 Curvas $\mathrm{P}$ e Q com $\Phi$ Constantes 30

3.3 Impedância da Carga no Máximo Carregamento 32

3.4 Limite de Estabilidade de Tensão 34

3.5 Índices para Avaliação da Estabilidade de Tensão 35

3.5.1 Método da Matriz [D'] 36

3.6 Sumário do Capítulo 38

4 Avaliação da Estabilidade de Tensão $\quad 39$

$\begin{array}{lll}4.1 & \text { Introdução } & 39\end{array}$

4.2 A Curva QV 40

4.3 Avaliação da Estabilidade de Tensão com a Curva QV 42

4.4 Obtendo o Índice $\left(\frac{\partial Q}{\partial V}\right) "$

4.5 Avaliação da Estabilidade de Tensão Utilizando o Índice $\left(\frac{\partial Q}{\partial V}\right) " \quad 46$

4.6 Incompatibilidade na Avaliação da Estabilidade de Tensão 46

$\begin{array}{lll}4.7 & \text { Sumário do Capítulo } & 47\end{array}$

$5 \quad$ Casos de Estudo $\quad 49$

5.1 Introdução 49

5.2 Estudo Comparativo do Índice $\left(\frac{\partial Q}{\partial V}\right)$ " e Margem da Curva QV 49

5.2.1 Estudos com Crescimento de Carga e Geração em uma Única Barra 50

5.2.1.1 Análise da Barra $1 \quad 51$

5.2.1.2 Análise da Barra $2 \quad 58$

5.2.1.3 Análise da Barra $4 \quad 62$

$\begin{array}{lll}\text { 5.2.1.4 Análise da Barra } 5 & 66\end{array}$

5.2.1.5 Conclusão 71

5.2.2 Estudos com Crescimento de Carga e Geração em Múltiplas Barras 71

5.2.2.1 Análise da Barra $1 \quad 72$

5.2.2.2 Análise da Barra $2 \quad 75$

$\begin{array}{lll}\text { 5.2.2.3 Análise da Barra } 4 & 77\end{array}$ 
$\begin{array}{lll}\text { 5.2.2.4 Análise da Barra } 5 & 80\end{array}$

5.2.2.5 Conclusão 82

5.3 Incongruência na Margem de Potência Reativa da Curva QV Obtida Através do Fluxo de Potência Continuado

5.3.1 Comparação das Cargas Máximas da Curva QV 83

5.3.2 Comparação da Margem da Curva QV 84

5.3.3 Margem da Curva QV para Diferentes Mecanismos de Aumento de Carga

5.3.4 Conclusão 93

$\begin{array}{ll}5.4 & \text { Sumário do Capítulo }\end{array}$

6 Conclusões $\quad 95$

$\begin{array}{lll}6.1 & \text { Trabalhos Futuros } & 96\end{array}$

$\begin{array}{lr}\text { Referências bibliográficas } & 97\end{array}$

A Dados de Sistema IEEE 14 Barras $\quad 99$ 


\section{Lista de figuras}

$\begin{array}{lll}\text { Figura 2.1 Barra Isolada } & 17\end{array}$

$\begin{array}{lll}\text { Figura 2.2 Sistemas de Duas Barras - modelo } \pi & 18\end{array}$

$\begin{array}{lll}\text { Figura 2.3 Correntes no Sistema de Duas Barras } & 19\end{array}$

Figura 3.1 Circuito de Duas Barras 28

Figura 3.2 Curvas $V_{1}$ Constante no Plano $\Theta V \quad 29$

Figura 3.3 Curvas $V_{1}$ Constante nos Eixos $\Theta_{1} \mathrm{VP} \quad 30$

Figura 3.4 $P_{1}$ e $Q_{1}$ Constantes no Plano $\Theta_{1} V_{1}$

Figura 3.5 Curvas P e Q com o mesmo Fator de Potência 32

Figura 3.6 Circuito com as Impedâncias da Transmissão e da Carga 33

Figura 3.7 Limite de Estabilidade de Tensão Sobre as Curvas de $\phi$ Constante no Plano SV 36

$\begin{array}{lll}\text { Figura 4.1 Curva QV } & 40\end{array}$

Figura 4.2 Curvas QV 42

Figura 5.1 Diagrama Unifilar do Sistema IEEE 14 Barras $\quad 50$

Figura 5.2 Índice $\left(\frac{\partial Q}{\partial V}\right)$ " na Barra $1 \quad 57$

Figura 5.3 Índice $\left(\frac{\partial Q}{\partial V}\right)$ " na Barra $2 \quad 62$

Figura 5.4 Índice $\left(\frac{\partial Q}{\partial V}\right)$ " na Barra $4 \quad 66$

Figura 5.5 Índice $\left(\frac{\partial Q}{\partial V}\right)$ " na Barra $5 \quad 71$

Figura 5.6 Índice $\left(\frac{\partial Q}{\partial V}\right)$ " na Barra $1 \quad 75$

$\begin{array}{lll}\text { Figura } 5.7 \text { índice }\left(\frac{\partial Q}{\partial V}\right) \text { " na Barra } 2 & 77\end{array}$

Figura 5.8 índice $\left(\frac{\partial Q}{\partial V}\right)$ " na Barra $4 \quad 80$

Figura 5.9 índice $\left(\frac{\partial Q}{\partial V}\right)$ " na Barra $5 \quad 82$

Figura A.1 Diagrama Unifilar do Sistema IEEE 14 Barras 99 


\section{Lista de tabelas}

Tabela 5.1 Análise da Barra 1 - Crescimento de Carga na Barra 252

Tabela 5.2 Tipos das Barras - Crescimento de Carga na Barra 256

Tabela 5.3 Análise da Barra 2 - Crescimento de Carga na Barra 258

Tabela 5.4 Análise da Barra 4 - Crescimento de Carga na Barra 263

Tabela 5.5 Análise da Barra 5 - Crescimento de Carga na Barra 267

Tabela 5.6 Análise na Barra 1 - Crescimento de Carga nas Barras 4 e 5

Tabela 5.7 Tipos das Barras - Crescimento de Carga nas Barras 4 e 574

Tabela 5.8 Análise na Barra 2 - Crescimento de Carga nas Barras 4 e 5

Tabela 5.9 Análise na Barra 4 - Crescimento de Carga nas Barras 4 e $5 \quad 78$

Tabela 5.10 Análise na Barra 5 - Crescimento de Carga nas Barras 4 e $5 \quad 80$

Tabela 5.11 Comparação das Cargas Máximas da Curva QV 84

Tabela 5.12 Casos do FPC com percentual de aumento de carga de 5\% 86

Tabela 5.13 Casos do FPC com percentual de aumento de carga de $10 \%$

Tabela 5.14 Cenário 1 das simulação de FPC com aumento de carga de $5 \%, 10 \%$ e $20 \%$

Tabela 5.15 Cenário 2 das simulação de FPC com aumento de carga de $5 \%, 10 \%$ e $20 \%$

Tabela 5.16 Cenário 3 das simulação de FPC com aumento de carga de $5 \%, 10 \%$ e $20 \%$

Tabela 5.17 Cenário 4 das simulação de FPC com aumento de carga de $5 \%, 10 \%$ e $20 \%$

Tabela 5.18 Cenário 5 das simulação de FPC com aumento de carga de $5 \%, 10 \%$ e $20 \%$

Tabela 5.19 Cenário 1 das versões do ANAREDE para aumento de carga do FPC com $5 \%$

Tabela 5.20 Cenário 2 das versões do ANAREDE para aumento de carga do FPC com $5 \%$

Tabela 5.21 Cenário 3 das versões do ANAREDE para aumento de carga do FPC com $5 \%$

Tabela 5.22 Cenário 1 das versões do ANAREDE para aumento de carga do FPC com $6.5 \%$

Tabela 5.23 Cenário 2 das versões do ANARinEDE para aumento de carga do FPC com $6.5 \%$

Tabela 5.24 Cenário 3 das versões do ANAREDE para aumento de carga do FPC com $6.5 \%$

Tabela 5.25 Cenário 4 das versões do ANAREDE para aumento de carga do FPC com $6.5 \%$

Tabela 5.26 Cenário 1 das versões do ANAREDE para aumento de carga do FPC com $10 \%$ 
Tabela 5.27 Cenário 2 das versões do ANAREDE para aumento de carga do FPC com $10 \%$

Tabela 5.28 Cenário 3 das versões do ANAREDE para aumento de carga do FPC com $10 \%$

Tabela 5.29 Cenário 1 das versões do ANAREDE para aumento de carga do FPC com $15 \%$

Tabela 5.30 Cenário 2 das versões do ANAREDE para aumento de carga do FPC com $15 \%$

Tabela 5.31 Cenário 3 das versões do ANAREDE para aumento de carga do FPC com $15 \%$ 


\section{Lista de Abreviaturas}

LEEA - Limite de Estabilidade Angular LET - Limite de Estabilidade de Tensão FCP - Fluxo de Potência Continuado SEE - Sistema de Energia Elétrica

CA - Corrente Alternada

LEEA - Limite de Estabilidade Angular

LET - Limite de Estabilidade de Tensão

FCP - Fluxo de Potência Continuado

SEE - Sistema de Energia Elétrica

CA - Corrente Alternada 


\section{Introdução}

\section{1}

\section{Considerações Gerais}

Nas últimas décadas diversos fatores têm levado ao aumento da probabilidade de ocorrência de blackouts em sistemas elétricos de grande porte, transformando o problema da estabilidade de tensão numa preocupação para diversos agentes do planejamento e operação do sistema elétrico [1].

O principal motivo que vem levando os sistemas elétrico de potência a operar perto dos limites de estabilidade é o aumento da demanda por energia elétrica, junto com o surgimento de novas restrições técnicas, econômicas e ambientais [2].

A sobrecarga da rede tende a ocorrer em momentos em que o sistema opera em condições de carga pesada, com elevado fluxo de potência ativa e reativa em suas linhas de transmissão, ou quando o sistema opera sob a perda de geradores ou linhas de transmissão.

Devido a importância de operar-se dentro da região normal, desenvolveram-se métodos que permitem prever e identificar os barramentos mais fracos e as áreas mais críticas do sistema. Diversos índices têm sido propostos para avaliar e prever o ponto de colapso de tensão [1].

\section{2}

\section{Objetivos}

O objetivo é realizar um estudo que apresente e contraponha as principais diferenças e semelhanças para dois índices que são utilizados para avaliação da estabilidade de tensão em sistemas elétricos.

A curva QV e sua margem são ferramentas amplamente utilizadas por engenheiros de operação e planejamento de sistema elétricos, sendo o primeiro índice estudado. Considera-se normal a operação quando o sistema opera com inclinação $\Delta V / \Delta Q$ positiva. A margem corresponde à distância entre o valor-base e o valor onde a inclinação troca de sinal. Procura-se operar sistemas elétricos sempre com uma margem 'grande', sendo motivo de preocupação se a inclinação é negativa ou se a margem é pequena [3]. 
O segundo índice estudado, parte do método da matriz $D^{\prime}$ e desenvolve a relação entre Q e V de cada barra através da relação $\left(\frac{\partial Q}{\partial V}\right)^{\prime \prime}=\operatorname{det}\left[D^{\prime}\right] /\left(\frac{\partial P}{\partial \Theta}\right)$.

Os dois índices são desenvolvidos e comparados em um estudo que procura demostrar possíveis divergências nesses índices quando operando próximo a sua carga máxima.

\section{3}

\section{Estrutura do Trabalho}

No Capítulo 2 apresentam-se conceitos relacionados ao problema de fluxo de potência, onde desenvolve-se suas equações junto com a formulação matricial do problema e aplicação do método numérico de Newton-Raphson para solucionar o problema.

No Capítulo 3 é abordado o problema da estabilidade de tensão, onde apresentam-se os conceitos de regiões de operação da curva $\phi$ constante no plano SV, dos limites de estabilidade estática angular e de estabilidade de tensão, a relação entre o uso de linhas de transmissão perto de sua capacidade máxima e os problemas de estabilidade de tensão. Também se apresenta uma ferramenta analítica de avaliação das condições nodais através do método da matriz [D'].

No Capítulo 4 é apresentado como obter-se a curva QV e como sua margem pode ser utilizada na avaliação da estabilidade de tensão. Um segundo índice também é desenvolvido através da matriz D' e, então, são explicitadas suas principais diferenças.

No Capítulo 5 apresentam-se aplicações práticas em um sistema teste dos índices apresentados no Capítulo 4. Assim são abordadas possíveis incoerências na avaliação da estabilidade de tensão ao utilizar o índice $\left(\frac{\partial Q}{\partial V}\right)$ " e a margem da curva QV em sistemas de potência próximos de seu carregamento máximo. Também foi discutido inconsistências no método de fluxo de potência continuado.

Finalmente, no Capítulo 6 apresentam-se as conclusões sobre o estudo comparativo em sistema com alto carregamento. Sugestões para futuros trabalhos também foram apresentadas. 


\section{2}

\section{Fluxo de Potência}

\section{1}

\section{Introdução}

O principal objetivo de um sistema de energia elétrica (SEE) de grande porte é atender a demanda de energia dos diversos consumidores do sistema, garantindo os requisitos mínimos de qualidade para o fornecimento de energia elétrica [4].

Além do equilíbrio entre potência gerada e consumida no SEE, existem outros requisitos econômicos e operacionais, como:

1. Manter os níveis de tensão nas barras dentro da faixa operativa;

2. Manter a frequência da rede dentro de determinada faixa especificada[5];

3. Operar com baixo nível de interrupção, permitindo que consumidores mantenham seus negócios em níveis adequados de confiabilidade;

4. Garantir o custo mínimo da energia, e a viabilidade econômica dos empreendimentos de geração e transmissão de energia elétrica.

Dentro desse contexto, o balanceamento de potência ativa em uma malha elétrica é de vital importância para o correto funcionamento do SEE, tendo em vista que a potência ativa de um sistema está diretamente relacionada a sua frequência operativa[5] e que a potência reativa se encontra diretamente relacionada com o módulo da tensão nas barras.

O fluxo de potência ou fluxo de carga consiste no estudo para determinar a tensão e ângulo nas barras, em junto com o fluxo de potência nos ramos. Essas informações são de grande utilidade no planejamento e expansão do SEE, mas também em sua operação.

Nesse capítulo será apresentada a teoria para execução do cálculo do fluxo de potência $\mathrm{CA}$ em sistemas de energia elétrica. A formulação das equações e suas premissas para modelagem fluxo de carga são desenvolvidas nas Seções 2.2 e 2.3. E finalmente, na Seção 2.4 é apresentado o método numérico de Newton-Raphson utilizado para resolver as equações não-lineares do fluxo de carga. 


\section{2}

\section{Equações de Carga}

As equações do problema do fluxo de carga são obtidas através da conservação da potência ativa e reativa nas barras do SEE.

Para o cálculo do fluxo de carga adota-se como premissa que as variações no tempo são suficientemente lentas, permitindo ignorar os efeitos transitórios do sistema [6]. Ou seja, o estudo de fluxo de carga é uma modelagem estática do sistema elétrico de potência.

Adota-se como referência que as potências injetadas na barra são positivas e que as potências que saem da barra são negativas.

A potência total injetada em uma barra é conhecida como potência aparente, (2.1), somatório de sua potência reativa e ativa. Analisando-se o sistema de uma única barra da Figura 2.1 obtêm-se (2.2).

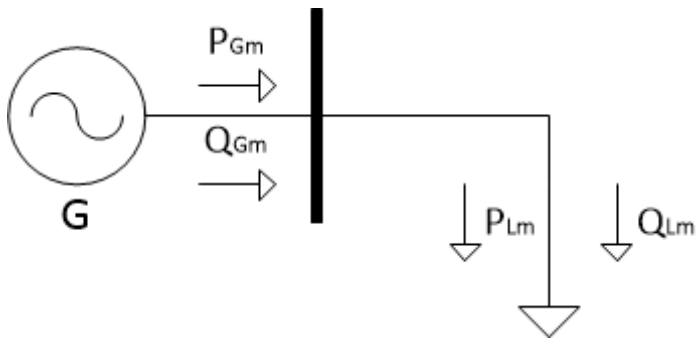

Figura 2.1: Barra Isolada

$$
\begin{gathered}
S=P+j Q \\
S=\left(P_{G}-P_{L}\right)+j\left(Q_{G}-Q_{L}\right)
\end{gathered}
$$

Para uma representação mais adequada do sistema elétrico de potência, o sistema de uma barra é expandido para um sistema de duas barras, conforme exibido na Figura 2.2.

As tensões nas barras, $V_{1}$ e $V_{2}$, são definidas por seu módulo e fase na barra:

$$
V_{1}=\left|V_{1}\right| e^{i \Theta_{1}} \quad \text { e } \quad V_{2}=\left|V_{2}\right| e^{i \Theta_{2}}
$$

A linha de transmissão é modelada através do modelo $\pi$ [7], composto por uma impedância em série (2.3), e duas admitâncias shunt em paralelo nas barras, (2.4):

$$
\begin{gathered}
Z=|Z| e^{j \alpha_{t}} \\
Y_{s h}=\left|V_{s h}\right| e^{j \alpha_{s h}}
\end{gathered}
$$


A potência aparente total injetada na barra é definida em termos da sua tensão e sua corrente conjugada:

$$
S=V * I^{*}
$$
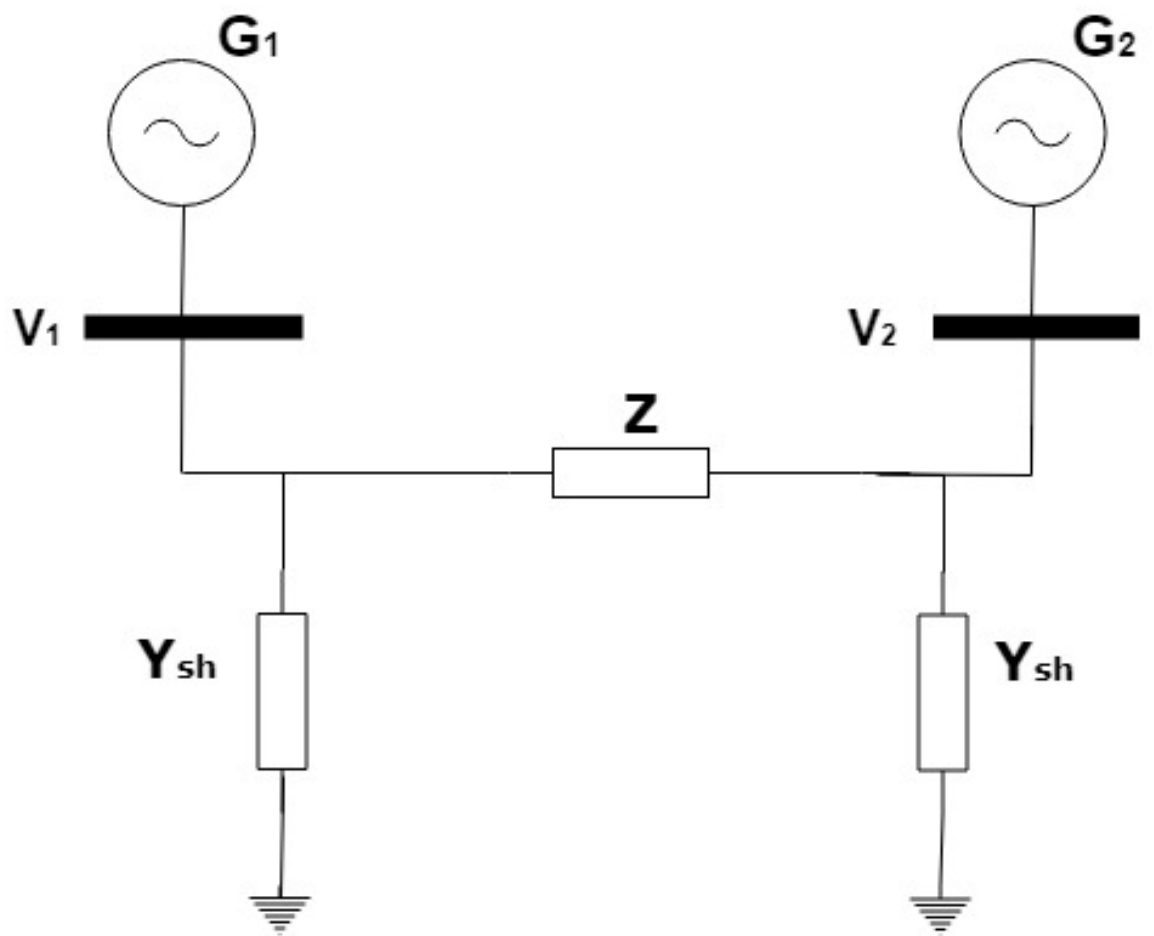

Figura 2.2: Sistemas de Duas Barras - modelo $\pi$

Na Figura 2.2 a corrente injetada na barra 1 é calculada como a soma da corrente na admitância shunt e na impedância em série:

$$
I_{1}=V_{1} \cdot Y_{s h}+\frac{\left(V_{1}-V_{2}\right)}{Z}
$$

Substituindo-se (2.6) em (2.5):

$$
S_{1}^{*}=\frac{V_{1} V_{1}^{*} j}{X c}+\frac{\left(V_{1}-V_{2}\right) V_{1}^{*}}{Z}
$$

Separando (2.7) em uma parte real e imaginária, obtêm-se:

$$
\begin{gathered}
P=-\frac{\left|V_{1}\right|^{2} \cos \left(\alpha_{t}\right)}{Z}+\frac{\left|V_{2}\right|\left|V_{1}\right| \cos \left(\Theta_{21}-\alpha_{t}\right)}{Z} \\
Q=\frac{\left|V_{1}\right|^{2}}{X_{c}}+\frac{\left|V_{1}\right|^{2} \sin \left(\alpha_{t}\right)}{Z}-\frac{\left|V_{2}\right|\left|V_{1}\right| \sin \left(\Theta_{21}-\alpha_{t}\right)}{Z}
\end{gathered}
$$

As equações (2.8) e (2.9) são as equações não-lineares do fluxo de carga, que serão solucionadas na Seção 2.4. 


\section{3}

\section{Desenvolvendo a Matriz de Admitância}

Sistemas elétricos podem ser muito mais complexos que um simples sistema de duas barras. Portanto, expande-se as equações de fluxo de carga, (2.8) e (2.9), tornando-se viável a modelagem computacional para um sistema de grande porte.

Modela-se o sistema de duas barras da Seção 2.2, porém para barras a e b genéricas.

Define-se que a corrente injetada na barra pode ser obtida pelo produto da matriz de corrente nodal versus a tensão na barra [7]:

$$
I=Y_{\text {nodal }} \cdot U
$$

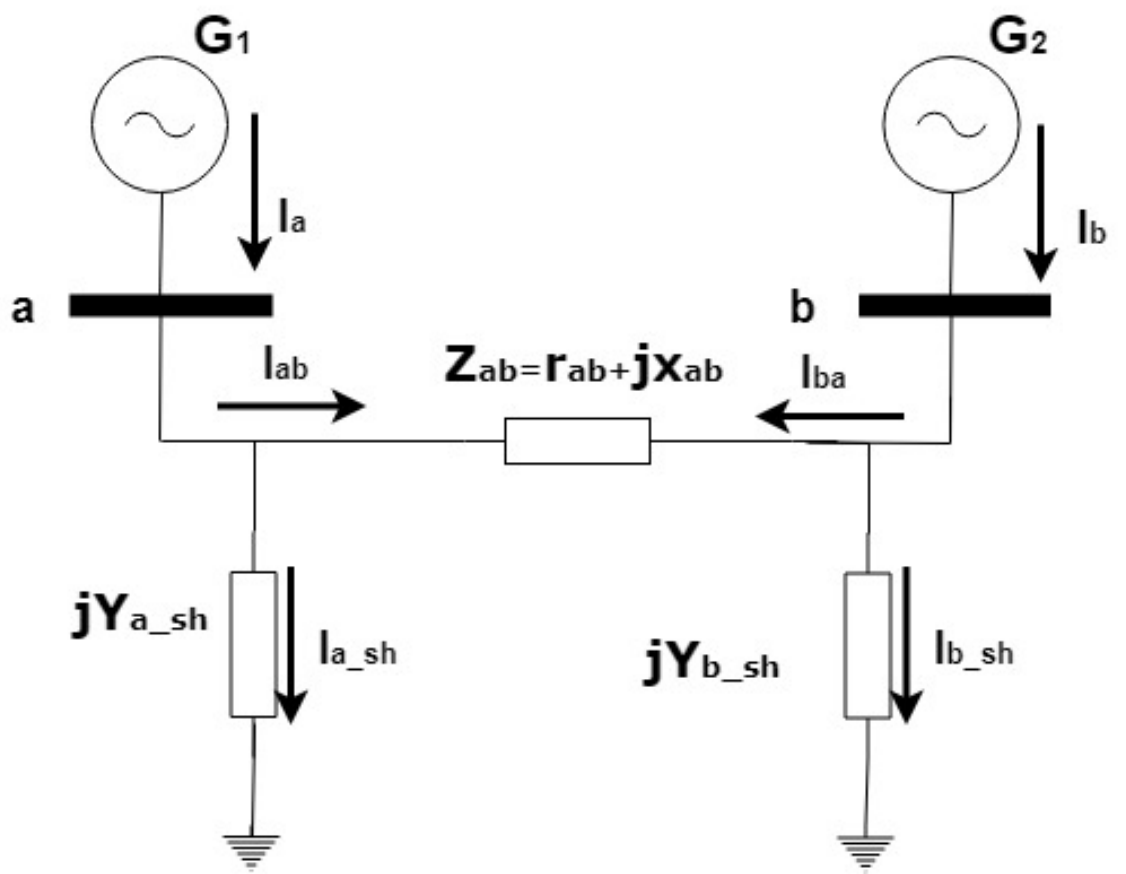

Figura 2.3: Correntes no Sistema de Duas Barras

A lei de Kirchhoff das correntes permite formular que a corrente injetada nas barras é igual a corrente que flui no elemento série da linha de transmissão mais a corrente sobre o elemento shunt, (2.10) e (2.11). E a admitância série da linha é definida por (2.12).

$$
\begin{gathered}
I_{a}=I_{a b}+I_{a_{s} h} \\
I_{b}=I_{b a}+I_{b_{s} h} \\
Y_{a b}=\frac{1}{Z_{a b}}=\frac{1}{R_{a b}+j X_{a b}}
\end{gathered}
$$


Já com a lei de Kirchhoff das tensões obtém-se que a corrente sobre o elemento série e shunt é obtida pela tensão na linha versus a admitância da linha.

$$
\begin{gathered}
I_{a b}=\left(\dot{U}_{a}-\dot{U}_{b}\right) \cdot Y_{a b} \\
I_{b a}=\left(\dot{U}_{b}-\dot{U}_{a}\right) \cdot Y_{a b} \\
I_{a-s h}=\dot{U}_{a} \cdot j Y_{a-s h} \\
I_{b-s h}=\dot{U}_{b} \cdot j Y_{b-s h}
\end{gathered}
$$

Substituindo-se (2.13), (2.14), (2.15) e (2.16) em (2.10) e (2.11) obtêm-se (2.17) em sua forma matricial:

$$
\left[\begin{array}{c}
I_{a} \\
I_{b}
\end{array}\right]=\left[\begin{array}{cc}
Y_{a b}+j Y_{a_{s} h} & -Y_{a b} \\
-Y_{a b} & Y_{a b}+j Y_{b_{s} h}
\end{array}\right]\left[\begin{array}{c}
U_{a} \\
U_{b}
\end{array}\right]
$$

A matriz $Y_{\text {nodal }}$ é importante no cálculo do fluxo de potência, sendo montada com as seguintes regras:

- O elemento da diagonal principal é a soma de todos os elementos que estão conectados a esse elemento (2.18);

- O elemento fora da diagonal principal representa a conexão entre a barra a e b, e define-se pelo negativo da admitância que conecta a barra a a barra b (2.19).

$$
\begin{gathered}
\mathbf{Y}_{\mathbf{a a}}=\sum_{b=1}^{n}\left(Y_{a b}+j Y_{a-s h}\right) \\
\mathbf{Y}_{\mathbf{a b}}=-Y_{a b}
\end{gathered}
$$

Separa-se a matriz nodal em duas partes: real e imaginária. A parte real é chamada de matriz de condutância nodal e a imaginária de matriz de suceptância nodal:

$$
\begin{gathered}
\mathbf{Y}_{\text {nodal }}=\left[\begin{array}{ccc}
Y_{11} & \cdots & Y_{1 a} \\
\cdots & \ddots & \vdots \\
Y_{a 1} & \cdots & Y_{a a}
\end{array}\right]=\left[\begin{array}{ccc}
G_{11}+j B_{11} & \cdots & G_{1 a}+j B_{1 a} \\
\cdots & \ddots & \vdots \\
G_{a 1}+j B_{a 1} & \cdots & G_{a a}+j B_{a a}
\end{array}\right] \\
\mathbf{Y}_{\text {nodal }}=\underbrace{\left[\begin{array}{ccc}
G_{11} & \cdots & G_{1 a} \\
\cdots & \ddots & \vdots \\
G_{a 1} & \cdots & G_{a a}
\end{array}\right]}_{\text {condutância }}+j \cdot \underbrace{\left[\begin{array}{ccc}
B_{11} & \cdots & B_{1 a} \\
\cdots & \ddots & \vdots \\
B_{a 1} & \cdots & B_{a a}
\end{array}\right]}_{\text {suceptância }}
\end{gathered}
$$


A potência e corrente injetada na barra já foram apresentadas na Seção 2.2 e são aqui desenvolvidas para as barras genéricas a e b:

$$
\begin{gathered}
S_{a}=\dot{U}_{a} \cdot \dot{I}_{a}^{*} \\
I_{a}=\sum_{a=1}^{n} Y_{a b} \cdot \dot{U}_{b}
\end{gathered}
$$

Substituindo-se (2.21) em (2.20):

$$
S_{a}=\dot{U}_{a} \cdot\left(\sum_{a=1}^{n} Y_{a b} \cdot \dot{U}_{b}\right)^{*}
$$

A tensão fasorial das barras a e b tem o seu módulo e ângulo apresentados em (2.23) e (2.24). E a matriz nodal em 2.3 apresenta duas parcelas, uma condutiva e outra susceptiva, que são desenvolvidas em (2.25).

$$
\begin{gathered}
\dot{U}_{a}=V_{a} \angle \Theta_{a}=V_{a} \cdot\left(\cos \Theta_{a}+j \sin \Theta_{a}\right) \\
\dot{U} b=V_{b} \angle \Theta_{b}=V_{b} \cdot\left(\cos \Theta_{b}+j \sin \Theta_{b}\right) \\
Y_{a b}=G_{a b}+j \cdot B_{a b}
\end{gathered}
$$

Finalmente, substituindo-se (2.23), (2.24) e (2.25) em (2.22) obtem-se (2.26).

$$
S_{a}=\left(\left(\sum_{b=1}^{n} G_{a b} \cdot V_{a} \cdot V_{b} \angle \Theta_{a b}\right)-j \cdot B_{a b} \cdot V_{b} \cdot V_{a} \angle \Theta_{a b}\right)
$$

A potência injetada na barra, (2.26), é dividida em parte real e imaginária, (2.27) e (2.28):

$$
\begin{gathered}
G=G_{a b} \cdot V_{b} \cdot V_{a} \cdot\left(\cos \Theta_{a b}+j \cdot \sin \Theta_{a b}\right) \\
B=-j \cdot B_{a b} \cdot V_{b} \cdot V_{a} \cdot\left(\cos \Theta_{a b}+j \cdot \sin \Theta_{a b}\right)
\end{gathered}
$$

Para finalizar, a potência aparente injetada na barra é apresentada em (2.29) para um sistema elétrico de $n$ barras, com suas parcelas de potência ativa e reativa apresentadas em (2.30) e (2.31).

$$
\begin{gathered}
S_{a}=\sum_{a=1}^{n}(G+B) \\
P_{a}=V_{a} \cdot \sum_{b=1}^{n}\left(V_{b} \cdot\left(G_{a b} \cdot \cos \Theta_{a b}+B_{a b} \cdot \sin \Theta_{a b}\right)\right), \forall a=1, \cdots, n \\
Q_{a}=V_{a} \cdot \sum_{m=1}^{n}\left(V_{a} \cdot\left(G_{a b} \cdot \sin \Theta_{a b}-B_{a b} \cdot \cos \Theta_{a b}\right)\right), \forall a=1, \cdots, n \\
\text { onde: }
\end{gathered}
$$

- n é o número de barras do sistema estudado; 
- $V_{a}$ e $V_{b}$ são os módulos das tensões nas barras do sistema genérico;

- $\Theta_{a}$ e $\Theta_{b}$ são os ângulos das tensões nas barras do sistema;

\section{4}

\section{Método de Newton-Raphson para Solução do Fluxo de Potência}

As equações do fluxo de carga (2.30) e (2.31) formam um sistema não-linear. Para resolver esse sistema é utilizado o método numérico de Newton-Raphson [6]. O método de Newton-Raphson consiste em realizar sucessivas aproximações até convergir e resolver as equações de carga.

Pode-se caracterizar cada barrar através de 4 variáveis:

- $V_{k}$ : módulo da tensão na barra k;

- $\theta_{k}$ : ângulo da tensão na barra k;

- $P_{k}$ : potência ativa líquida injetada na barra $\mathrm{k}$;

- $Q_{k}$ : potência reativa líquida injetada na barra k;

Essas variáveis são divididas em dois tipos: variáveis de controle e variáveis de estado. As variáveis de controle são as dadas e as variáveis de estado são aquelas a serem calculadas.

As barras são classificadas e modeladas levando em consideram o seu papel no funcionamento do SEE. Assim, definem-se os seguintes tipos de barra:

- Barra de carga ou PQ: Não há controle de tensão nas barras e não possuem geração. Conhecem-se as variáveis $P_{k}$ e $Q_{k}$, e calculam-se $V_{k}$ e $\theta_{k}$.

- Barra de tensão controlada ou PV: Possuem dispositivos de controle que permitem manter o módulo de tensão e a injeção de potência ativa dentro da faixa de valores especificados. Conhecem-se as variáveis $P_{k} \mathrm{e}$ $V_{k}$, e calculam-se $Q_{k}$ e $\theta_{k}$.

- Barra de referência, swing ou V $\theta$ : Na maioria das referências ([7], [4]) esses três tipos de barras são consideradas como do mesmo tipo. De maneira genérica, é a barra que fornece a referência angular do sistema, fechando o balanço de perdas de potência ativa do sistema. Conhecem-se as variáveis $V_{k}$ e $\theta_{k}$, e calculam-se $P_{k}$ e $Q_{k}$

Porém, as barras swing e de referência angular não são necessariamente as mesmas. A barra swing tem o papel de compensar as perdas de potência ativa. Uma barra swing tem $\mathrm{P}$ livre para variar, isto é, dentro dos limites configurados. Uma barra swing pode ou não ter V controlada. Por outro lado, a barra de referência angular é a barra com ângulo de referência fixo, normalmente igual a zero. 
- Barra de controle de tensão ou P: Possui Q variável, é utilizada para controle da tensão em uma barra remota. Conhecem-se as variáveis $P_{k}$, e calculam-se $Q_{k}, V_{k}$ e $\theta_{k}$.

- Barra remota ou PQV: É uma barra de carga que tem sua tensão controlada remotamente por uma ou mais barras $\mathrm{P}$. O controle remoto de tensão pode ser efetuado por um ou mais taps variáveis. Conhecem-se as variáveis $P_{k}, Q_{k}$ e $V_{K}$, calculam-se $\theta_{k}$.

A primeira etapa para resolução das equações de fluxo de potência consiste em calcular $\mathrm{V}$ e $\theta$ para todas as barras do sistema. Portanto, pretende-se calcular $V_{k}$ e $\theta_{k}$ nas barras PQ e $\theta_{k}$ nas barras PV.

Após conhecer $V_{k}$ e $\theta_{k}$ para todas as barras da rede, a segunda etapa consiste em calcular $P_{k}$ e $Q_{k}$ para a barra $\mathrm{V} \theta$, e $Q_{k}$ para as barras $\mathrm{PV}$, assim como outras variáveis de interesse. É importante ressaltar que a barra V $\theta$ é responsável por fechar o balanço de perdas do sistema.

O método de Newton-Raphson modela e soluciona um sistema de equações composto por duas equações e duas incógnitas para cada barra PQ e uma equação e uma incógnita para cada barra PV.

Com o objetivo de modelar o sistema algébrico e resolver o fluxo de potência nas barras PQ e PV, escreve-se uma equação para P especificado:

$$
\Delta P_{k}=P_{k}^{e s p}-P_{k}=0
$$

E para as barras PQ, escreve-se uma equação para Q especificado:

$$
\Delta Q_{k}=Q_{k}^{e s p}-Q_{k}=0
$$

$P_{k}^{e s p}$ e $Q_{k}^{e s p}$ são as potências ativas e reativas especificadas na barra k, e $P_{k}$ e $Q_{k}$ são as potências ativas e reativas injetadas nas barras, dados por (2.30) e (2.31).

As equações (2.32) e (2.33) são reescritas em sua forma vetorial, (2.34) e (2.35):

$$
\begin{aligned}
& \Delta P=P^{e s p}-P(V, \theta) \\
& \Delta Q=Q^{e s p}-Q(V, \theta)
\end{aligned}
$$

Defini-se a função vetorial $\mathrm{g}(\mathrm{x})$ :

$$
g(x)=\left[\begin{array}{l}
\Delta \bar{P} \\
\Delta \bar{Q}
\end{array}\right]
$$

As variáveis a calcular-se são:

$$
x=\left[\begin{array}{c}
\bar{\theta} \\
\bar{V}
\end{array}\right]
$$


O método de Newton-Raphson consiste em calcular novos valores de $P_{k}$ e $Q_{k}$ até obter-se $g(x)=0$.

Portanto, pretende-se determinar os valores de $\theta_{k}$ e $V_{k}$ para quando a potência ativa e reativa $P_{k}$ e $Q_{k}$ é idêntica à potência ativa e reativa especificada, resultando em $g(x)=0$.

O problema de obter-se $x$ que resulta em $g(x)=0$ é solucionado pelo método de Newton-Raphson com o seguinte passo a passo:

1. Define-se um contador de iterações $\mathrm{i}=0$ e escolhe-se $\theta_{k}^{(0)}$ inicial para as barras PQ e PV e $V_{k}^{(0)}$ inicial para as barras PQ.

2. Com $\theta_{k}^{(0)}$ e $V_{k}^{(0)}$ iniciais, calcula-se $P_{k}^{(i)}$ para as barras PQ e PV e $Q_{k}^{(i)}$ para as barras PQ, e calcula-se os resíduos $\Delta P_{k}^{(i)}$ e $\Delta Q_{k}^{(i)}$.

3. Compara-se $g\left(x^{(i)}\right)$ com a tolerância $\varepsilon$. Caso, $\max \left|\Delta P_{k}^{(i)}\right|<\varepsilon \mathrm{e}$ $\max \left|\Delta Q_{k}^{(i)}\right|<\varepsilon$, o método de Newton-Raphson convergiu e a solução $\theta_{k}^{(i)}$ e $V_{k}^{(i)}$ foi encontrada. Quando a condição de convergência não é atingida, passa-se para o próximo passo.

4. Lineariza-se $g\left(x^{(i)}\right)$ em torno do ponto $\left(x^{(i)} ; g\left(x^{(i)}\right)\right)$ por intermédio da série de Taylor, desprezando-se os termos de ordem maior que 1:

$$
g\left(x^{(i)}+\Delta x^{(i)}\right) \simeq g\left(x^{(i)}\right)+g^{\prime}\left(x^{(i)}\right) \Delta x
$$

sendo $g^{\prime}(x)$ a derivada de primeira ordem, assim $g^{\prime}(x)=\frac{\mathrm{d} g}{\mathrm{~d} x}$.

Finalmente, ao linearizar (2.38) para solucionar o fluxo de carga, define-se a matriz Jacobiano como:

$$
J^{(i)}=-\left[\begin{array}{ll}
\frac{\partial P}{\partial \Theta} & \frac{\partial P}{\partial V} \\
\frac{\partial Q}{\partial \Theta} & \frac{\partial Q}{\partial V}
\end{array}\right]=\left[\begin{array}{ll}
H & N \\
M & L
\end{array}\right]
$$

A matriz Jacobiana é dividida em quatro subgrupos ou sub-matrizes:

- H: derivada parcial da potência ativa com relação a $\Theta$;

- L: derivada parcial da potência ativa com relação a V;

- M: derivada parcial da potência reativa com relação a $\Theta$;

- N: derivada parcial da potência reativa com relação a $\mathrm{V}$.

Os elementos das sub-matrizes H, N, M e L são dados por:

$$
\begin{gathered}
H_{l m}=\frac{\partial P_{l}}{\partial \Theta_{m}}=V_{l} \cdot V_{m} \cdot\left(G_{l m} \sin \theta_{l m}-B_{l m} \cos \theta_{l m}\right) \\
H_{l l}=\frac{\partial P_{l}}{\partial \Theta_{l}}=-V_{l}^{2} \cdot B_{k k}-V_{l} \cdot \sum_{m=1}^{n} V_{m} \cdot\left(G_{l m} \sin \theta_{l m}-B_{l m} \cos \theta_{l m}\right)
\end{gathered}
$$




$$
\begin{gathered}
N_{l m}=\frac{\partial P_{l}}{\partial V_{m}}=V_{l} \cdot\left(G_{l m} \cos \theta_{l m}+B_{l m} \sin \theta_{l m}\right) \\
N_{l l}=\frac{\partial P_{l}}{\partial V_{l}}=V_{l} \cdot G_{l l}-V_{l} \cdot \sum_{m=1}^{n} V_{m} \cdot\left(G_{l m} \sin \theta_{l m}-B_{l m} \cos \theta_{l m}\right) \\
M_{l m}=\frac{\partial Q_{l}}{\partial \Theta_{m}}=-V_{l} \cdot V_{m} \cdot\left(G_{l m} \cos \theta_{l m}-B_{l m} \sin \theta_{l m}\right) \\
M_{l l}=\frac{\partial Q_{l}}{\partial \Theta_{l}}=-V_{l}^{2} \cdot G_{l l}+V_{l} \cdot \sum_{m=1}^{n} V_{m} \cdot\left(G_{l m} \cos \theta_{l m}+B_{l m} \sin \theta_{l m}\right) \\
L_{l m}=\frac{\partial Q_{l}}{\partial V_{m}}=V_{l} \cdot\left(G_{l m} \sin \theta_{l m}-B_{l m} \cos \theta_{l m}\right) \\
L_{l l}=\frac{\partial Q_{l}}{\partial \Theta_{l}}=-V_{l} \cdot B_{l l}+V_{l} \cdot \sum_{m=1}^{n} V_{m} \cdot\left(G_{l m} \sin \theta_{l m}-B_{l m} \cos \theta_{l m}\right)
\end{gathered}
$$

5. Ao linearizar $g\left(x^{(i)}\right)$, obtêm-se:

$$
g\left(x^{(i)}\right)+g^{\prime}\left(x^{(i)}\right) \Delta x=0
$$

que é reescrito de uma forma mais conveniente:

$$
g\left(x^{(i)}\right)=-g^{\prime}\left(x^{(i)}\right) \Delta x
$$

Rearranjando-se (2.40) e substituindo-se a matriz Jacobiano, (2.36) e (2.37). Resulta no seguinte sistema linear:

$$
\left[\begin{array}{c}
\Delta P \\
\Delta Q
\end{array}\right]=\left[\begin{array}{ll}
\frac{\partial P}{\partial \Theta} & \frac{\partial P}{\partial V} \\
\frac{\partial Q}{\partial \Theta} & \frac{\partial Q}{\partial V}
\end{array}\right]_{J}\left[\begin{array}{c}
\Delta \Theta \\
\Delta V
\end{array}\right]
$$

onde:

- J é a matriz Jacobiana, aplicando a linearização de Taylor em (2.30) e (2.31), consistindo nas derivadas parciais de $\mathrm{P}$ e Q com relação a $\mathrm{V}$ e $\Theta$.

- $\Delta P$ é o resíduo de potência ativa em todas as barras do sistema com exceção das barras do tipo $\mathrm{V} \Theta$, onde não se define P. Portanto, as equações relativas a $\mathrm{P}$ serão excluídas do sistema (2.41).

- $\Delta Q$ é o resíduo de potência reativa em todas as barras do sistema com exceção das barras do tipo PV e V $\Theta$, onde não se define Q. Portanto, as equações relativas a Q serão excluídas do sistema (2.41).

- $\Delta \Theta$ é o resíduo do ângulo da tensão nas barras.

- $\Delta V$ é o resíduo do módulo da tensão nas barras. 
6. Determinam-se novos resíduos, fazendo-se:

$$
\theta^{(i+1)}=\theta^{(i)}+\Delta \theta^{(i)} \text { e } V^{(i+1)}=V^{(i)}+\Delta V^{(i)}
$$

7. Faz-se $i=i+1$ e retorna-se ao passo 2 .

\section{5}

\section{Sumário do Capítulo}

Nesse capítulo foi apresentado o método numérico de Newton-Raphson, junto com o desenvolvimento das equações de fluxo de carga, para solucionar-se as equações de fluxo de carga do sistema elétrico de potência.

O fluxo de potência em conjunto com a matriz Jacobiana podem ser utilizados como instrumentos para obtenção de índices de estabilidade de tensão.

Esses índices são tema de estudo do presente trabalho e são apresentados nos Capítulos 3 e 4. 


\section{3}

\section{Estabilidade de Tensão}

O conceito de estabilidade de tensão é definido pela capacidade que um sistema elétrico de potência possui em manter suas barras operando sob as condições normais de operação, mesmo quando submetido a distúrbios da rede [2].

Na ocorrência de distúrbios como aumento da carga ou perda da geração, o sistema pode passar a operar fora da região usual, nesse caso, ações de controle podem ter resultados contrários ao esperado.

Tradicionalmente, o problema da estabilidade de tensão tem sua origem no alto carregamento das linhas de transmissão e conseguinte aumento da compensação de potência reativa[2].

Um dos principais objetivos do estudo de estabilidade de tensão é identificar quando o sistema de potência passa a operar fora da região usual de operação. Nesse capítulo são introduzidos os conceitos iniciais de estabilidade de tensão e o limite de estabilidade angular (LEEA) para um sistema simplificado de duas barras nas Seções 3.1 e 3.2. Na Seção 3.3 desenvolve-se a impedância da carga no máximo carregamento do sistema e na Seção 3.4 apresenta-se o lugar geométrico formado pelo conjunto de máximos carregamentos e limite de estabilidade de tensão (LET) do sistema. E, finalmente, na Seção 3.5 apresenta-se um modelo matemático simples para avaliar, utilizando-se a matriz Jacobiano, em qual região de operação o SEE encontra-se.

\section{1}

\section{Límite de Estabilidade Estática Angular}

Para o estudo do limite de estabilidade "estática"angular é calculado o limite máximo do carregamento de potência ativa para um sistema de duas barras [8], Figura 3.1.

O sistema exemplo de duas barras é composto por um gerador com capacidade infinita de geração, uma carga com potência constante e uma linha de transmissão sem limite térmico. Para simplificar o estudo, as admitâncias shunt da linha de transmissão são desprezadas, sem perda de generalidade. 


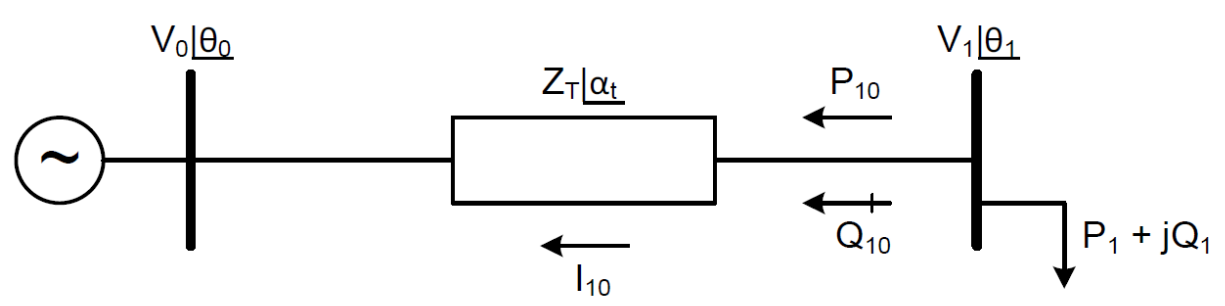

Figura 3.1: Circuito de Duas Barras

O estudo foi desenvolvido para um sistema de potência com os seguintes parâmetros:

$$
\begin{gathered}
V_{0}=1 \mathrm{pu} \\
\theta_{0}=0^{\circ} \\
Z_{t}=0,2 \mathrm{pu} \\
\alpha=70^{\circ} \\
-\infty<P_{0}<+\infty \\
-\infty<Q_{0}<+\infty
\end{gathered}
$$

Na Figura 3.1, o fluxo da potência aparente partindo da carga é dado por:

$$
S_{10}^{*}=P_{10}-j Q_{10}=V_{10}^{*} I_{10}
$$

A corrente da barra 1 para barra 0 e a tensão conjugada na barra 1 são definidas como em:

$$
\begin{gathered}
I_{10}=\frac{V_{1} \angle \theta_{1}-V_{0} \angle \theta_{0}}{Z_{t} \angle \alpha_{t}} \\
V_{1}^{*}=V_{1} \angle-\theta_{1}
\end{gathered}
$$

Portanto, é possível substituir (3.2) e (3.3) em (3.1) obtendo-se (3.4), que pode ser desmembrado em parte real (3.5) e imaginária (3.6).

$$
\begin{gathered}
S_{10}^{*}=\left[\frac{V_{1}^{2} \cos \left(\alpha_{t}\right)}{Z_{t}}-\frac{V_{1} V_{0} \cos \left(\theta_{10}+\alpha_{t}\right)}{Z_{t}}\right]-j\left[\frac{V_{1}^{2} \sin \left(\alpha_{t}\right)}{Z_{t}}-\frac{V_{1} V_{0} \sin \left(\theta_{10}+\alpha_{t}\right)}{Z_{t}}\right] \\
P_{10}=-P_{1}=\frac{V_{1}^{2} \cos \left(\alpha_{t}\right)}{Z_{t}}-\frac{V_{1} V_{0} \cos \left(\theta_{10}+\alpha_{t}\right)}{Z t} \\
Q_{10}=-Q_{1}=\frac{V_{1}^{2} \operatorname{sen}\left(\alpha_{t}\right)}{Z_{t}}-\frac{V_{1} V_{0} \operatorname{sen}\left(\theta_{10}+\alpha_{t}\right)}{Z_{t}}
\end{gathered}
$$

O objetivo é determinar a potência máxima que pode ser entregue à carga. Considera-se $V_{1}$ constante em (3.5), traçando-se a curva da potência consumida na carga em função do ângulo da tensão na barra 1, Figura 3.2. 
Na Figura 3.2 apresentam-se curvas $V_{1}$ constante no plano $\Theta V$ para diversos valores de $V_{1}$. Observa-se que para todas as curvas traçadas, com $V_{1}$ constante, existe uma máxima potência ativa que pode ser transmitida à carga, quando $\theta_{1}=-\alpha_{t}$.

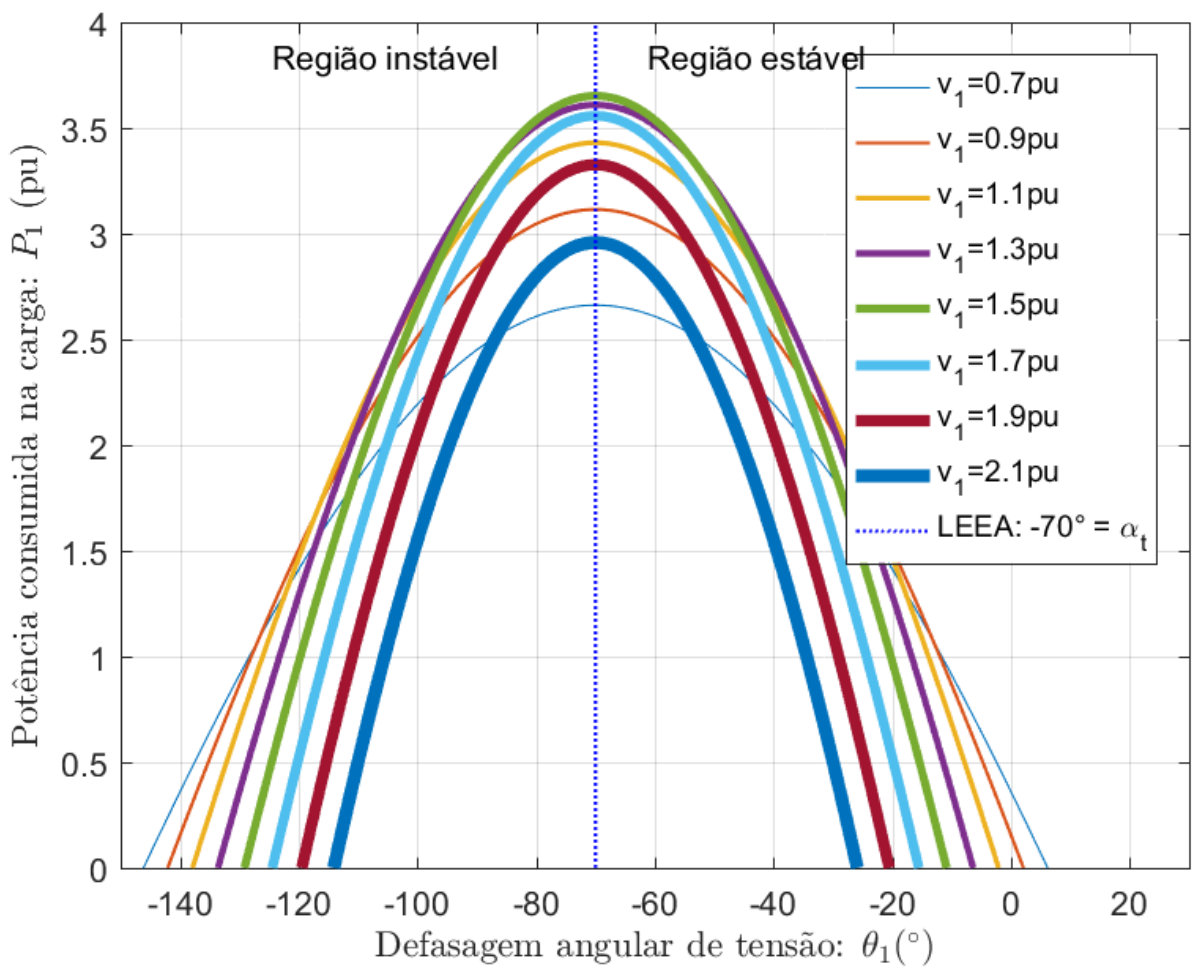

Figura 3.2: Curvas $V_{1}$ Constante no Plano $\Theta V$

Ao observar a relação entre $V_{1}$ e $P_{1}$ na Figura 3.2, percebe-se que a medida que $V_{1}$ aumenta o valor máximo de $P_{1}$ aumenta. Entretanto, a partir de certo valor de $V_{1}$, à medida que $V_{1}$ aumenta, o valor máximo de $P_{1}$ decresce. Portanto, existe uma potência ativa máxima que pode ser transmitida para a carga, mesmo com capacidade infinita de compensação de potência reativa na barra de carga. Essa potência máxima é conhecida como potência "maximum maximorum" [8].

A potência "maximum maximorum" pode ser observada com facilidade na Figura 3.3. A reta definida por $\theta_{1}=-\alpha_{t}$, determina o limite de estabilidade "estática"angular (LEEA), e pode ser observada nas Figuras 3.2 e 3.3 [8]. 


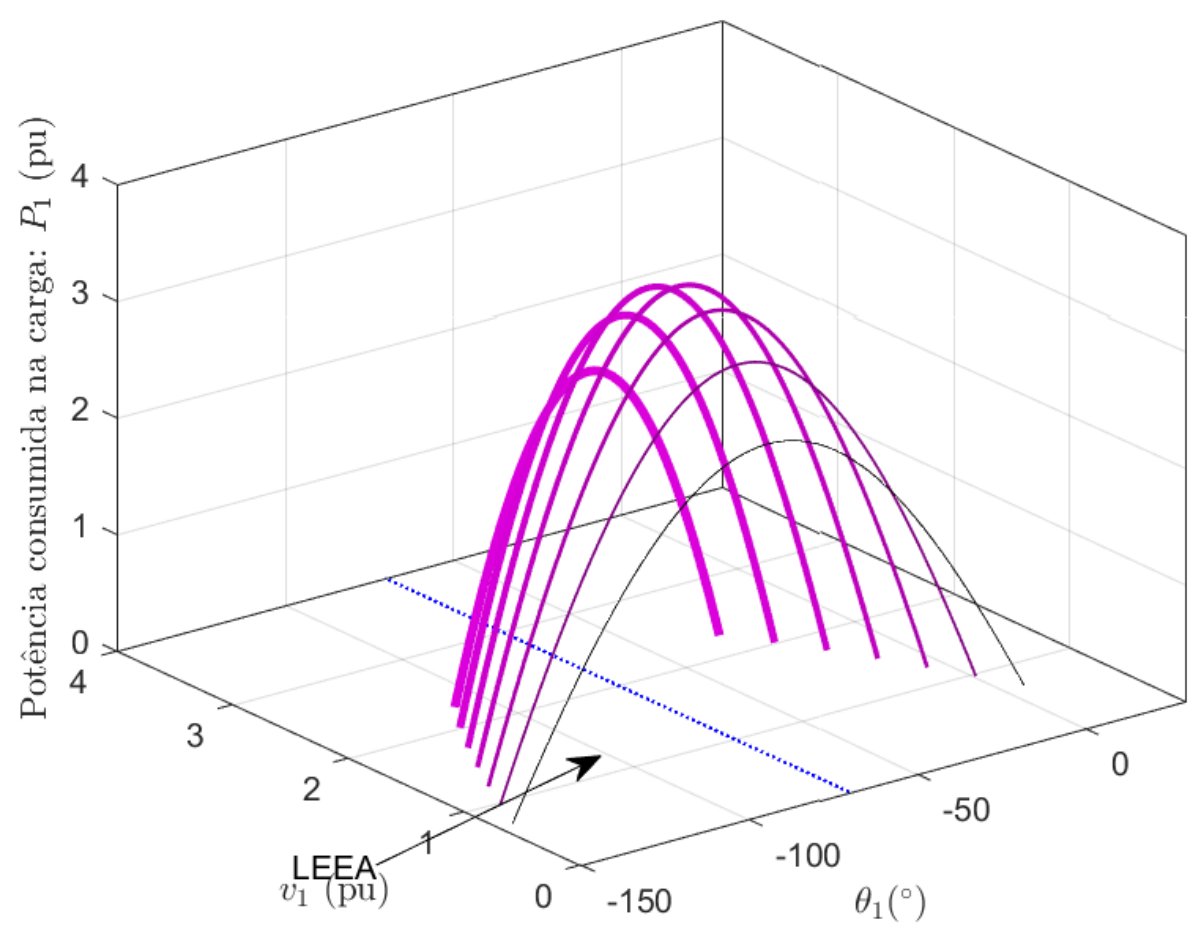

Figura 3.3: Curvas $V_{1}$ Constante nos Eixos $\Theta_{1}$ VP

\section{2}

\section{Curvas $\mathbf{P}$ e $\mathbf{Q}$ com $\Phi$ Constantes}

O objetivo desta seção é avaliar o aumento do carregamento do sistema de duas barras, Figura 3.1, e definir a existência uma máxima carga ativa.

Reescrevendo-se (3.5) e (3.6) para vizualizar uma equação quadrática em função de $V_{1}$, obtêm-se:

$$
\begin{aligned}
& V_{1}^{2}\left[\frac{\cos \left(\alpha_{t}\right)}{Z_{t}}\right]-V_{1}\left[\frac{V_{0} \cos \left(\theta_{10}+\alpha_{t}\right)}{Z_{t}}\right]+P_{1}=0 \\
& V_{1}^{2}\left[\frac{\sin \left(\alpha_{t}\right)}{Z_{t}}\right]-V_{1}\left[\frac{V_{0} \sin \left(\theta_{10}+\alpha_{t}\right)}{Z_{t}}\right]+Q_{1}=0
\end{aligned}
$$

Da mesma forma como foram traçadas curvas no plano $\Theta P$ na Figura 3.2, pode-se traçar curvas no plano $\Theta V$. Fazendo-se $P_{1}$ constante e variando $\theta_{1}$ em (3.7), pode-se calcular $V_{1}$ e, portanto, traçar a curva $P_{1}$ constante no plano $\Theta V$. Analogamente, variando-se $\theta_{1}$ em (3.8), pode-se calcular $V_{1}$ e, assim traçar a curva $Q_{1}$ constante no plano $\Theta V$.

Na Figura 3.4, apresentam-se as curvas de nível para quatro valores de $P_{1}$ e $Q_{1}$.

Na Figura 3.4 observa-se a existência de uma máxima carga ativa ("maximum maximorum"), mesmo com compensação ilimitada de potência 


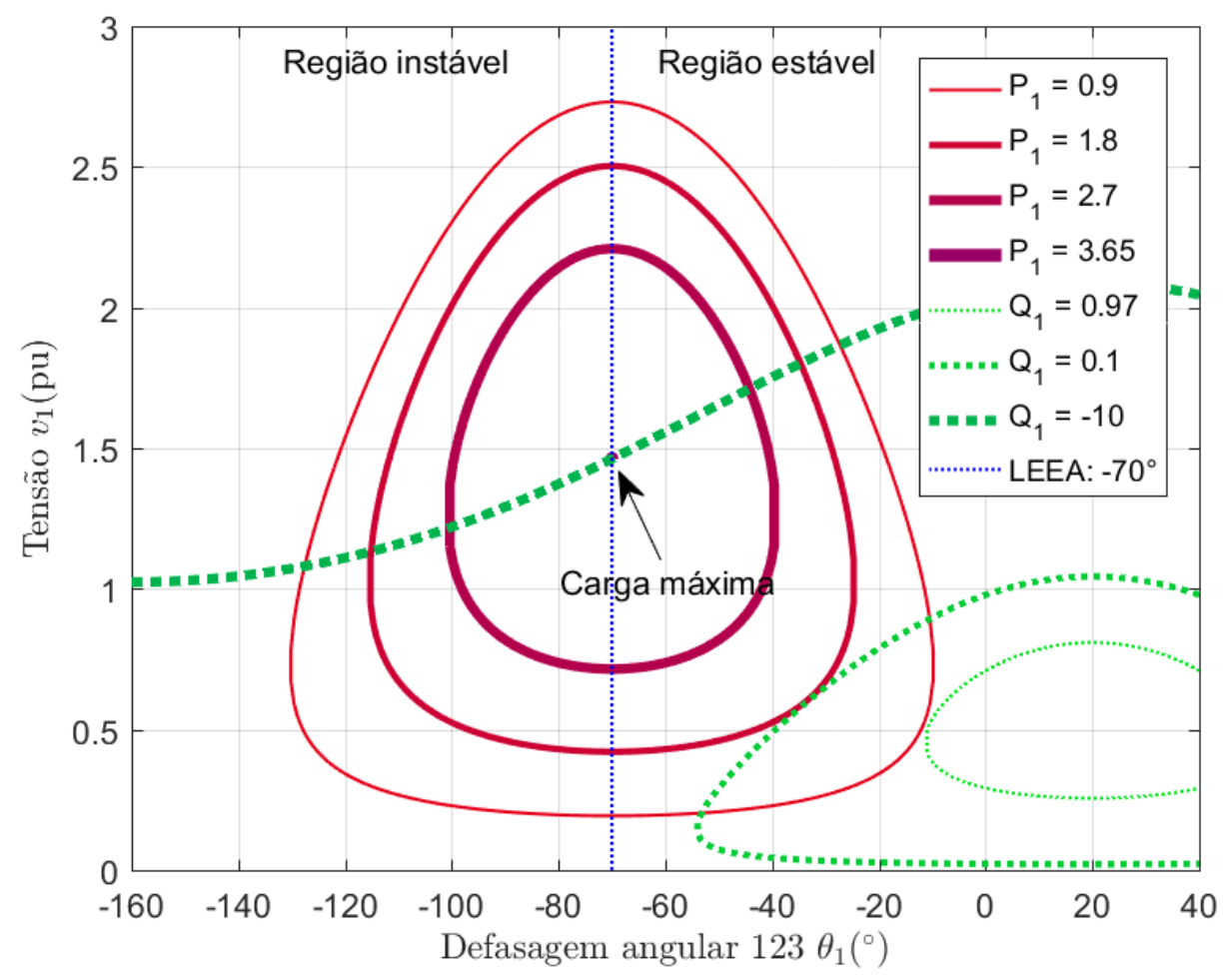

Figura 3.4: $P_{1}$ e $Q_{1}$ Constantes no Plano $\Theta_{1} V_{1}$

reativa. Nesse sistema de duas barras, a carga máxima é obtida para $P_{1}=3,65$ MW e $Q_{1}=-10,00$ Mvar.

Percebe-se uma correspondência entre as curvas das Figuras 3.2, 3.3 e 3.4, com todas indicando a existência de uma máxima potência que pode ser transmitida para carga.

Assim, os pontos de operação formados por pares $\theta_{1} V_{1} \operatorname{com} \theta_{1}=-\alpha_{t}$ definem o lugar geométrico chamado de LEEA. O LEEA é obtido fazendo-se $V_{1}$ constante e calculando o ponto de máximo em (3.5).

Ao traçar as curvas no plano $\Theta V$ para diferentes valores de $\mathrm{P}$ e Q, porém, mantendo-se o fator de potência $\phi$ constante, um melhor entendimento do comportamento do sistema frente ao aumento da capacidade de transmissão de carga é obtido.

$\mathrm{Na}$ Figura 3.5 apresenta-se novamente $\mathrm{P}$ e $\mathrm{Q}$ constantes, como na Figura 3.4, mais a curva $\phi$ constante no plano $\Theta V$, variando-se $\theta_{1}$ em (3.9) e calculando-se $V_{1}$.

$$
V_{1}=\frac{V_{0}\left[\sin \left(\theta_{01}+\alpha_{t}\right)-\tan (\phi) \cos \left(\theta_{01}+\alpha_{t}\right)\right]}{\sin \left(\alpha_{t}\right)-\tan (\phi) \cos \left(\alpha_{t}\right)}
$$

Analisando-se as curvas da Figura 3.5, verifica-se que para a carga, $P_{a}+j Q_{a}$, pode-se ter duas soluções de tensão: $V_{a}$ e $V_{a}^{\prime}$ (interseção das curvas $P_{a}$ e $\left.Q_{a}\right)$. 


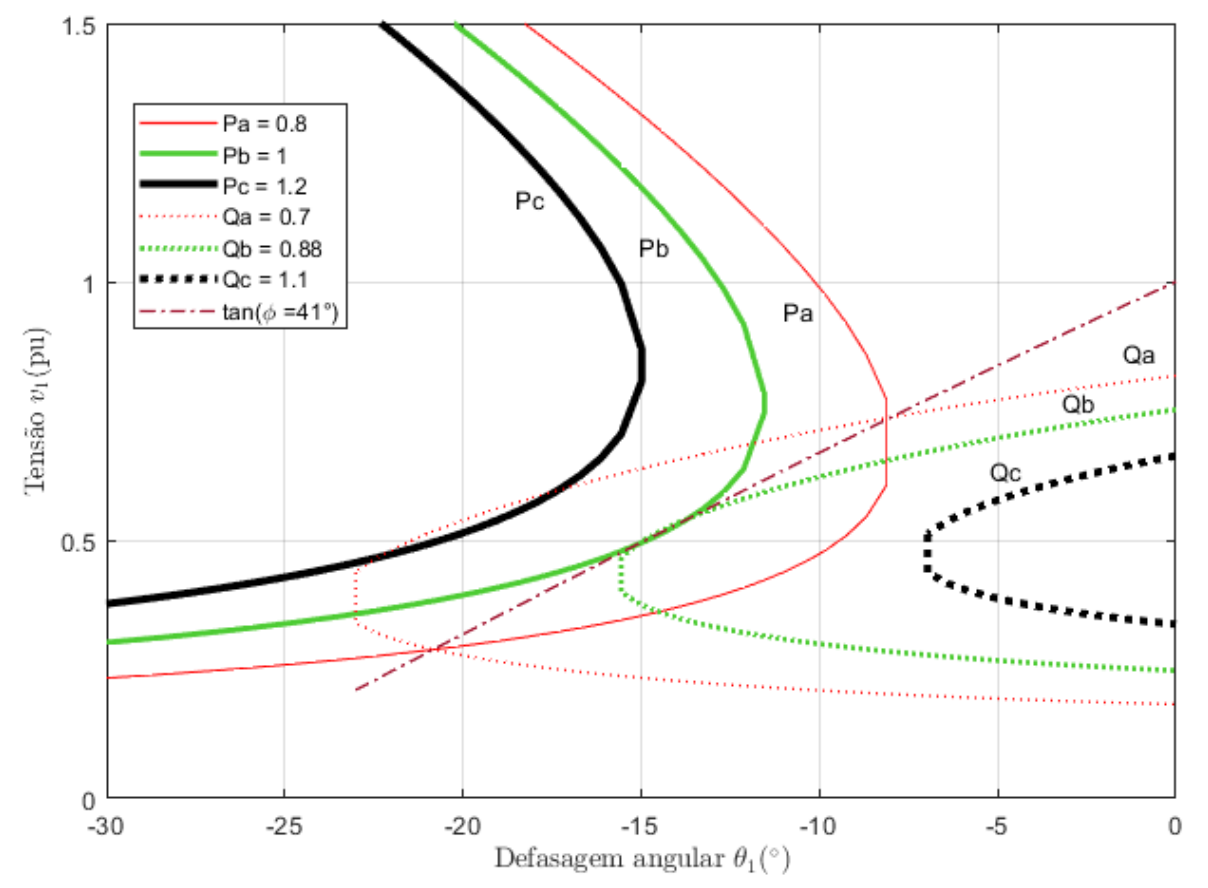

Figura 3.5: Curvas P e Q com o mesmo Fator de Potência

Aumentando-se a carga para $P_{b}+j Q_{b}$, mantendo-se o fator de potência constante, as duas soluções de tensão $V_{b}$ e $V_{b}^{\prime}$ aproximam-se até coincidirem em um único ponto $V_{b}=V_{b}^{\prime}$, interseção das curvas $P_{b}$ e $Q_{b}$.

Assim, conclui-se que se $P$ e $Q$ continuarem aumentando, a curva $P_{c}$ constante no plano $\theta_{1} V_{1}$ e $Q_{c}$ constante no plano $\theta_{1} V_{1}$ não mais se cruzam, ou seja, não há solução para a tensão. Desta forma, para certo fator de potência $\phi$, há uma máxima carga ativa e reativa que pode ser alimentada.

Portanto, pode-se verificar a existência de duas, uma ou nenhuma solução para a tensão ao aumentar o carregamento do sistema. Quando há duas soluções para a tensão em uma barra do sistema, uma delas pertencerá à região normal de operação e a outra à região anormal de operação, onde ações de controle de tensão podem ter efeito oposto ao esperado [8].

\section{3}

\section{Impedância da Carga no Máximo Carregamento}

A máxima potência que pode ser transmitida para uma carga, para cada fator de potência, está relacionada ao valor de sua impedância equivalente. Será demostrado que no ponto de máxima potência a impedância da carga é igual à impedância da linha de transmissão ("casamento de impedância"). 


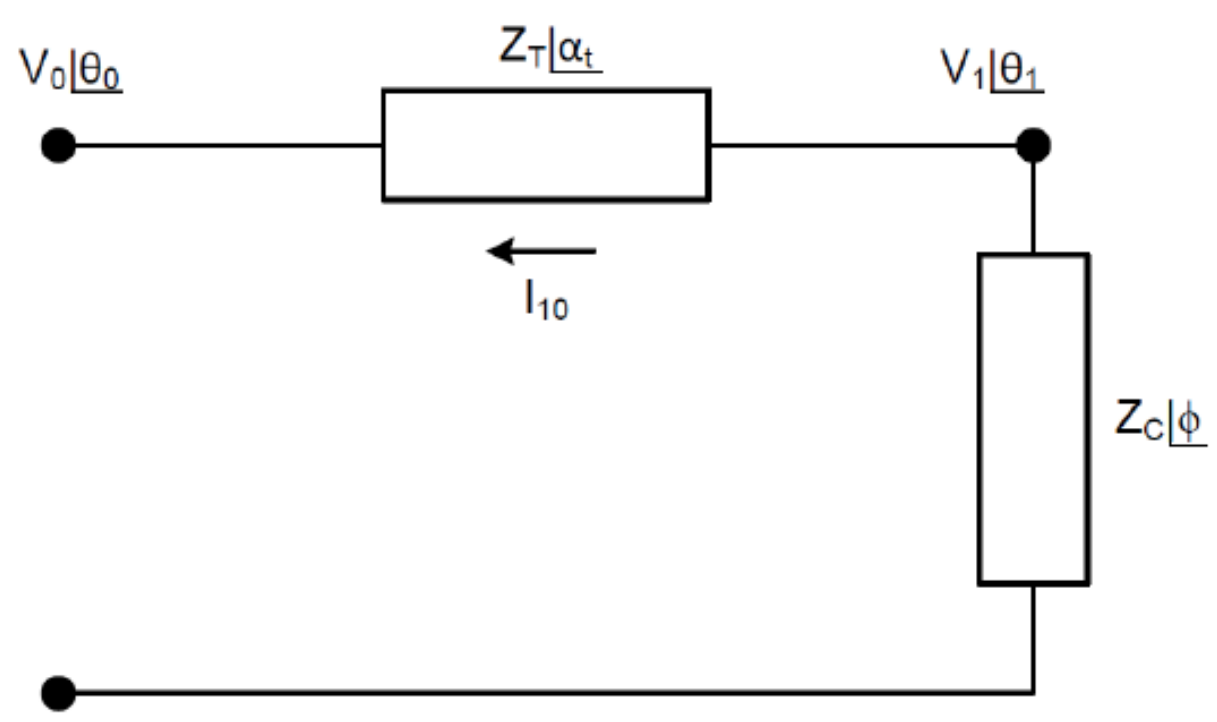

Figura 3.6: Circuito com as Impedâncias da Transmissão e da Carga

Na Figura 3.6, a corrente que flui para a carga é:

$$
I_{01}=\frac{V_{0}}{Z_{t} \angle \alpha_{t}+Z_{C} \angle \phi}
$$

Logo:

$$
I_{01}=\frac{V_{0}}{\sqrt{\left(Z_{t} \cos \left(\alpha_{t}\right)+Z_{c} \cos (\phi)\right)^{2}+\left(Z_{t} \sin \left(\alpha_{t}\right)+Z_{c} \sin (\phi)\right)^{2}}}
$$

Por outro lado:

$$
P_{10}=-P_{1}=-I_{01}^{2} Z_{C} \cos (\phi)
$$

Substituindo-se (3.10) em (3.11):

$$
P_{10}=-\frac{V_{0}^{2} Z_{C} \cos (\phi)}{Z_{t}^{2} \cos ^{2}\left(\alpha_{t}\right)+2 Z_{t} Z_{C} \cos \left(\alpha_{t}\right) \cos (\phi)+Z_{C}^{2} \cos ^{2}(\phi)+Z_{t}^{2} \sin ^{2}\left(\alpha_{t}\right)+2 Z_{t} Z_{C} \sin \left(\alpha_{t}\right) \sin (\phi)+Z_{C}^{2} \cos ^{2}(\phi)}
$$

Aplicando-se a propriedade trigonométrica do cosseno da diferença em (3.12), é possível reescrever:

$$
P_{10}=-P 1=\frac{V_{0}^{2} Z_{C} \cos (\phi)}{Z_{t}^{2}+Z_{C}^{2}+2 Z_{t} Z_{C} \cos \left(\alpha_{t}-\phi\right)}
$$

Como o objetivo é obter a impedância de carga no máximo carregamento:

$$
\frac{\partial P_{1}}{\partial Z_{C}}=\frac{V_{0}^{2} \cos (\phi)\left[Z_{t}^{2}+Z_{C}^{2}+2 Z_{t} Z_{C} \cos \left(\alpha_{t}-\phi\right)\right]}{\left[Z_{t}^{2}+Z_{C}^{2}+2 Z_{t} Z_{C} \cos \left(\alpha_{t}-\phi\right)\right]^{2}}-\frac{V_{0}^{2} Z_{C} \cos (\phi)\left[2 Z_{C}+2 Z_{t} \cos \left(\alpha_{t}-\phi\right)\right]}{\left[Z_{t}^{2}+Z_{C}^{2}+2 Z_{t} Z_{C} \cos \left(\alpha_{t}-\phi\right)\right]^{2}}=0
$$

Rearranjando-se (3.14) obtêm-se: 


$$
V_{0}^{2} Z_{t}^{2} \cos (\phi)=V_{0}^{2} Z_{C}^{2} \cos (\phi) \therefore Z_{C}=Z_{t}
$$

O segundo passo na análise de (3.13) é avaliar se o ponto de inflexão encontrado em (3.15) é um ponto de máximo ou mínimo. Faz-se a segunda derivada da potência injetada igual a zero em 3.16. Como a segunda derivada com $Z_{c}=Z_{t}$ é menor que zero, conclui-se que quando $Z_{c}=Z_{t}, P_{1}$ é máximo.

$$
\frac{\partial^{2} P_{1}}{\partial Z_{C}^{2}}=0
$$

\section{4}

\section{Limite de Estabilidade de Tensão}

O conjunto dos pontos de operação no máximo carregamento define o lugar geométrico chamado de limite de estabilidade de tensão (LET). Partindo de (3.13), chega-se a uma relação analítica que permite identificar o LET.

Para o ponto de máximo carregamento, o módulo da impedância da carga é igual ao módulo da impedância da linha de transmissão. Substituindo-se (3.15) em (3.13), obtém-se:

$$
P_{10}^{\min }=\frac{V_{0}^{2} Z_{C} \cos (\phi)}{2 Z_{C}^{2}\left[1+\cos \left(\alpha_{t}-\phi\right)\right]}=-\frac{V_{0}^{2} \cos (\phi)}{4 Z_{C} \cos ^{2}\left(\frac{\alpha_{t}-\phi}{2}\right)}
$$

A carga máxima é então:

$$
P_{1}^{\max }=\frac{V_{0}^{2} \cos (\phi)}{4 Z_{C} \cos ^{2}\left(\frac{\alpha_{t}-\phi}{2}\right)}
$$

A tensão na barra 1 é calculada como o produto da impedância da carga pela corrente:

$$
V_{1}=Z_{C} I_{01}
$$

Logo:

$$
V_{1}=\frac{V_{0} Z_{C}}{\sqrt{2 Z_{t}^{2}\left(1+\cos \left(\alpha_{t}-\phi\right)\right)}} \therefore V_{1}=\frac{V_{0}}{\sqrt{4 \cos ^{2} \frac{\left(\alpha_{t}-\phi\right)}{2}}}
$$

Assim, a tensão no ponto de máximo carregamento é:

$$
V_{1}=\frac{V_{0}}{2 \cos \left(\frac{\alpha_{t}-\phi}{2}\right)}
$$

A tensão na barra 1, pode ser escrita como:

$$
\dot{V}_{1}=\dot{Z}_{C} \dot{I_{01}} \therefore V_{1} \angle \theta_{1}=Z_{C} \angle \phi \frac{\dot{V}_{0}}{Z_{t} \angle \alpha_{t}+Z_{C} \angle \phi}
$$


Expandindo-se (3.18) e considerando-se apenas a parte real de $\dot{V}_{1}$, obtém-se:

$$
V_{1} \cos \left(\theta_{1}\right)=\frac{V_{0}\left[\cos \left(\alpha_{t}\right) \cos (\phi)+\cos ^{2}(\phi)+\sin \left(\alpha_{t}\right) \sin (\phi)+\sin ^{2}(\phi)\right]}{\cos ^{2}\left(\alpha_{t}\right)+2 \cos \left(\alpha_{t}\right)+\cos ^{2}(\phi)+\sin ^{2}\left(\alpha_{t}\right)+2 \sin \left(\alpha_{t}\right)+\sin ^{2}(\phi)}
$$

Reduzindo e simplificando (3.19), obtém-se:

$$
V_{1}=\frac{V_{0}}{2 \cos \left(\theta_{1}\right)}
$$

Igualando-se (3.17) e (3.20), o ângulo na barra 1, no ponto de máximo carregamento é:

$$
\theta_{1}=\frac{\alpha_{t}-\phi}{2}
$$

A máxima transmissão de potência à carga, para cada fator de potência, define o LET. Em outras palavras, variando-se $\phi$ e utilizando-se (3.20) e (3.21) traça-se o LET sobre as curvas de $\phi$ constante no plano SV, conforme Figura 3.7 .

Na Figura 3.7 mostra-se diferentes curvas no plano SV, uma para cada fator de potência $\phi$ e a curva do LET passando por todos os pontos de máximo carregamento. As curvas no plano SV com fator de potência constante são também conhecidas como curvas do nariz [2].

O LET passa pelas "pontas do nariz" de todas as curvas para $\phi$ constante no plano SV, isto é, une todos os pontos de máximo carregamento. Além disso, o LET separa as duas regiões de operação: região superior da curva $\phi$ constante no plano SV, onde se tem controle sobre a tensão e região inferior da curva para $\phi$ constante no plano SV, onde as ações de controle de tensão podem ter efeito oposto ao esperado [2].

\section{5}

\section{Índices para Avaliação da Estabilidade de Tensão}

A operação na parte inferior da curva SV não é considerada normal. Portanto, torna-se importante métodos para avaliação em tempo real se a barra opera na parte inferior ou superior da curva SV.

Assim, são desenvolvidos índices de estabilidade de tensão que representam a margem operativa do sistema [1], para cálculo em tempo real. Desenvolveu-se, portanto, uma ferramenta analítica de avaliação das condições nodais associadas ao máximo fluxo de potência ativa e reativa de uma rede de transmissão.

Baseado em um modelo matemático simples e de direta interpretação física do fenômeno, são deduzidos índices abrangentes e significativos para 


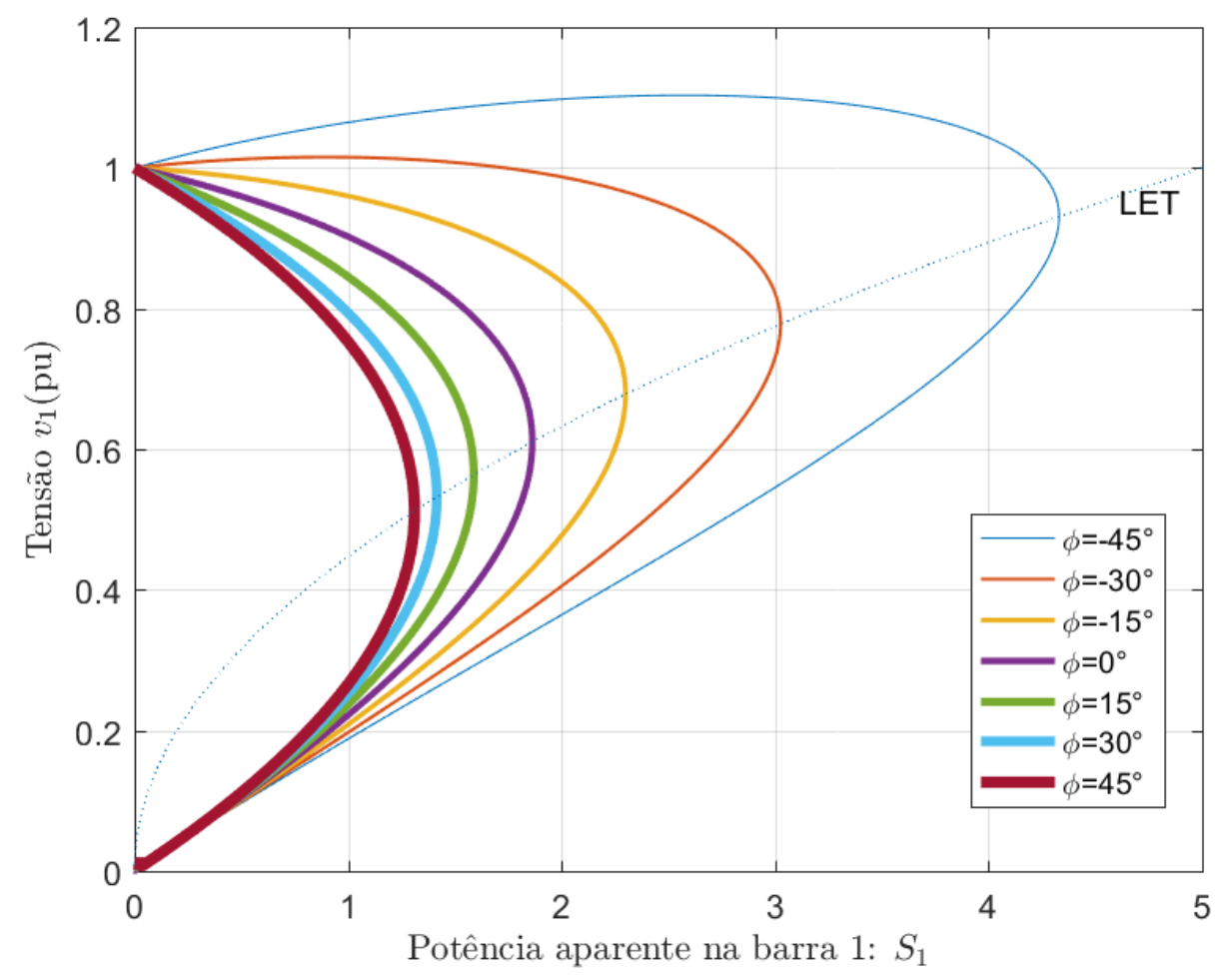

Figura 3.7: Limite de Estabilidade de Tensão Sobre as Curvas de $\phi$ Constante no Plano SV

avaliação da região de operação da curva $\phi$ constante no plano SV.

\subsection{1}

\section{Método da Matriz [D']}

O objetivo é ter-se uma ferramenta que permita identificar se a barra i opera na região superior ou inferior da curva $\phi$ constante no plano SV, ou no caso do plano VQ, do lado direito ou esquerdo.

Considera-se inicialmente a barra em análise como do tipo PQ. O sistema linearizado das equações estáticas de fluxo de potência, (2.39), é reescrito com o deslocamento para baixo das equações referentes a barra i e para a direita das colunas referentes as suas variáveis: 


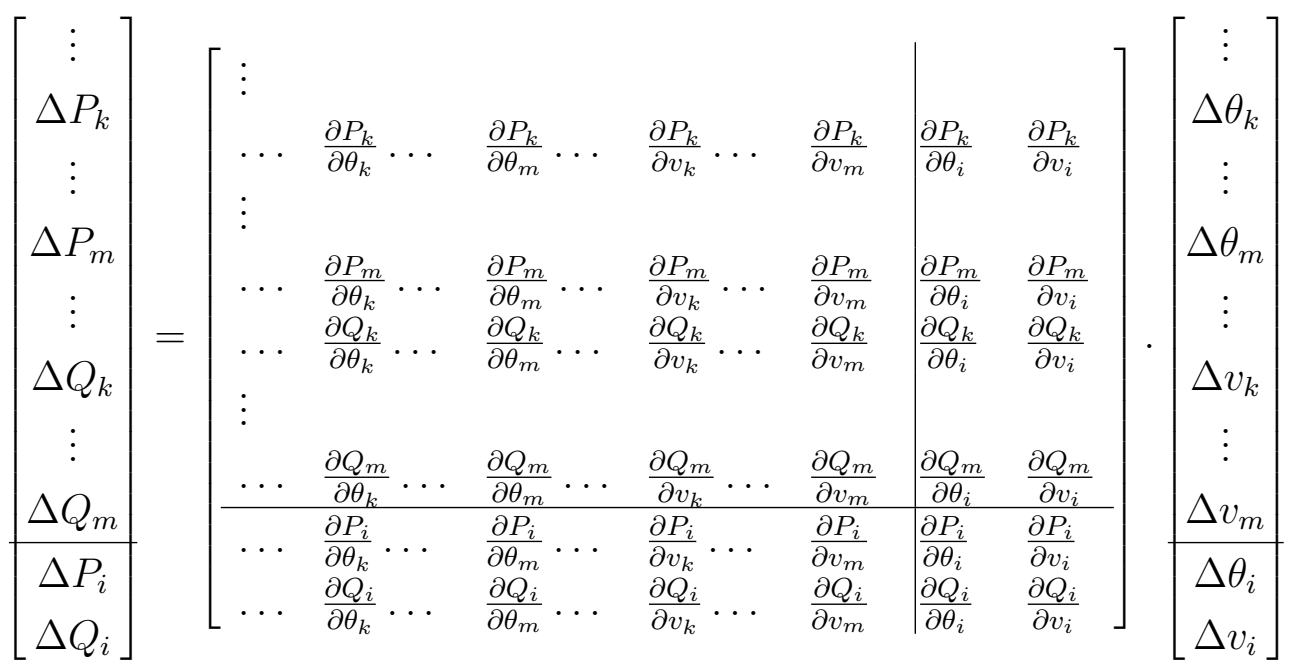

A matriz Jacobiano em (3.22) é, portanto, dividida em quatro submatrizes A, B, C e D, como em (3.23).

$$
\left[\begin{array}{l}
\Delta P^{\prime} \\
\Delta Q^{\prime} \\
\Delta P_{i} \\
\Delta Q_{i}
\end{array}\right]=\left[\begin{array}{ll}
A & B \\
\hline C & D
\end{array}\right]\left[\begin{array}{c}
\Delta \theta^{\prime} \\
\Delta v^{\prime} \\
\Delta \theta_{i} \\
\Delta v_{i}
\end{array}\right]
$$

O objetivo de um estudo de estabilidade de tensão nas barras de um sistema elétrico de potência é identificar o efeito de aumentos de carga.

Portanto, estudam-se variações infinitesimais em $\Delta P_{i}$ e $\Delta Q_{i}$ e seus efeitos em $\Delta \Theta_{i}$ e $\Delta V_{i}$. Para avaliar as variações de $\mathrm{P}$ e $\mathrm{Q}$ na barra i é necessário fixar $\mathrm{P}$ e $\mathrm{Q}$ nas demais barras do sistema e assim faz-se $\Delta P^{\prime}=\Delta Q^{\prime}=0$ :

$$
\left[\begin{array}{c}
0 \\
0 \\
\Delta P_{i} \\
\Delta Q_{i}
\end{array}\right]=\left[\begin{array}{ll}
A & B \\
\hline C & D
\end{array}\right]\left[\begin{array}{c}
\Delta \theta^{\prime} \\
\Delta v^{\prime} \\
\Delta \theta_{i} \\
\Delta v_{i}
\end{array}\right]
$$

Ao desenvolver algebricamente (3.24), obtêm-se a relação de potência ativa e reativa injetadas com módulo e ângulo da tensão na barra i em (3.25).

$$
\left[\begin{array}{c}
\Delta P_{i} \\
\Delta Q_{i}
\end{array}\right]=\left[D-C A^{-1} B\right]\left[\begin{array}{c}
\Delta \theta_{i} \\
\Delta v_{i}
\end{array}\right]
$$

A matriz [D'] é definida por:

$$
\left[D^{\prime}\right]=\left[D-C A^{-1} B\right]
$$

Em trabalhos anteriores [1] e em diversos estudos da área de estabilidade é demonstrado que o módulo e o sinal do determinante da matriz $\left[D^{\prime}\right]$ indicam respetivamente a distância do ponto de máximo carregamento e a região da curva $\phi$ constante no plano SV. 
No presente estudo, porém, o método da matriz $\left[D^{\prime}\right]$ será utilizado como base para desenvolver uma relação de sensibilidade QV ou VQ.

\section{6}

\section{Sumário do Capítulo}

Nesse capítulo foi apresentado de maneira analítica e numérica, o limite da estabilidade de tensão para um sistema de duas barras.

Para esse sistema foi determinada a máxima potência que pode ser entregue a carga e sua relação com o LET.

O LET permite desenvolver o conceito de duas regiões de operação para o sistema de potência.

Foi introduzido o índice de estabilidade de tensão baseado na matriz [D'] que será discutido em maior profundidade no Capítulo 4 para desenvolvimento do índice $\left(\frac{\partial Q}{\partial V}\right)^{"}$. 


\section{4}

\section{Avaliação da Estabilidade de Tensão}

\section{1}

\section{Introdução}

O problema de estabilidade de tensão é motivo de preocupação das equipes de operação e planejamento de sistemas elétrico de potência. Assim, cada vez mais engenheiros estão utilizando ferramentas que levam em consideração o problema da estabilidade de tensão para operar e planejar o sistema de potência.

Este capítulo aborda dois índices com aplicação na análise da estabilidade de tensão: a curva QV e o índice $\left(\frac{\partial Q}{\partial V}\right)^{\prime}$. E discorre sobre como obter esses índices, sua aplicabilidade, e possíveis incoerências no estudo da estabilidade de tensão.

Nessa dissertação, almeja-se explicitar as premissas utilizadas na obtenção desses índices e, consequentemente, analisar suas similaridades e diferenças. Em alguns casos, a avaliação de operação na região normal obtida com um desses índices pode divergir com relação ao outro índice. Essas prováveis divergências serão investigadas.

A curva QV é um gráfico formado pela tensão de uma barra i no eixo $\mathrm{x}$ das abscissas e pela potência reativa absorvida ou gerada na barra i no eixo y das ordenadas. Em 2000, Chowdhury e Taylor publicaram um artigo abordando o uso a curva QV frente a técnicas de simulação dinâmica de longo prazo [9].

A nomenclatura natural para este gráfico seria curva VQ, como pode ser encontrado na literarura [9]. Porém, nesse trabalho será adotado o nome de "curva QV", utilizado pelo software ANAREDE do CEPEL.

O método da curva QV permite estimar, em determinada barra i do sistema, a potência reativa gerada ou absorvida (Q), para determinada tensão $(\mathrm{V})$. Este recurso oferece um panorama do desempenho do sistema durante eventos que possam comprometer a margem de potência reativa.

Um segundo método para avaliar a estabilidade de tensão abordado nesse trabalho consiste em utilizar a matriz D' [1]. Dessa matriz obtém-se o índice $\left(\frac{\partial Q}{\partial V}\right)^{\prime}$, índice que representa a sensibilidade QV em uma barra em um ponto 
de operação. Em 1992, Gao, Baofu, Morison e Kundur publicaram um artigo abordando o uso da análise modal para avaliar a estabilidade de tensão [10] a partir da matriz QV.

Nesse capítulo será discutido o procedimento para obtenção da curva QV na Seção 4.2 e sua utilização para avaliar a estabilidade de sistemas de potência na Seção 4.3. O procedimento para obtenção do índice $\left(\frac{\partial Q}{\partial V}\right)$ " é apresentado e discutido na Seção 4.4, enquando que sua aplicabilidade na avaliação de sistema de potência é abordada na Seção 4.5. E finalmente, na Seção 4.6 são apresentadas as possíveis diferenças nos índices $\left(\frac{\partial Q}{\partial V}\right) "$ e lado da curva QV.

\section{2}

\section{A Curva QV}

A curva QV é obtida com a resolução de uma sucessão de problemas de fluxos de potência. Varia-se a tensão na barra i em torno da tensão no caso base. Cada alteração da tensão consiste em um novo caso, com uma nova tensão e potência reativa. Anota-se, portanto, o valor da tensão e potência reativa gerada ou absorvida para todos os casos obtidos.

A barra i passa a ser modelada com um compensador síncrono fictício acoplado e do ponto de vista da modelagem computacional, a barra é representada por uma barra do tipo PV sem limite de potência reativa.

Na Figura 4.1 exibe-se a curva QV, onde é observado o ponto de operação no caso base e no caso de inflexão. O ponto de fronteira da curva está localizado no ponto de potência reativa mínima que divide a curva entre os lados direito e esquerdo. O lado direito é o usual de operação; V e Q variam no mesmo sentido.

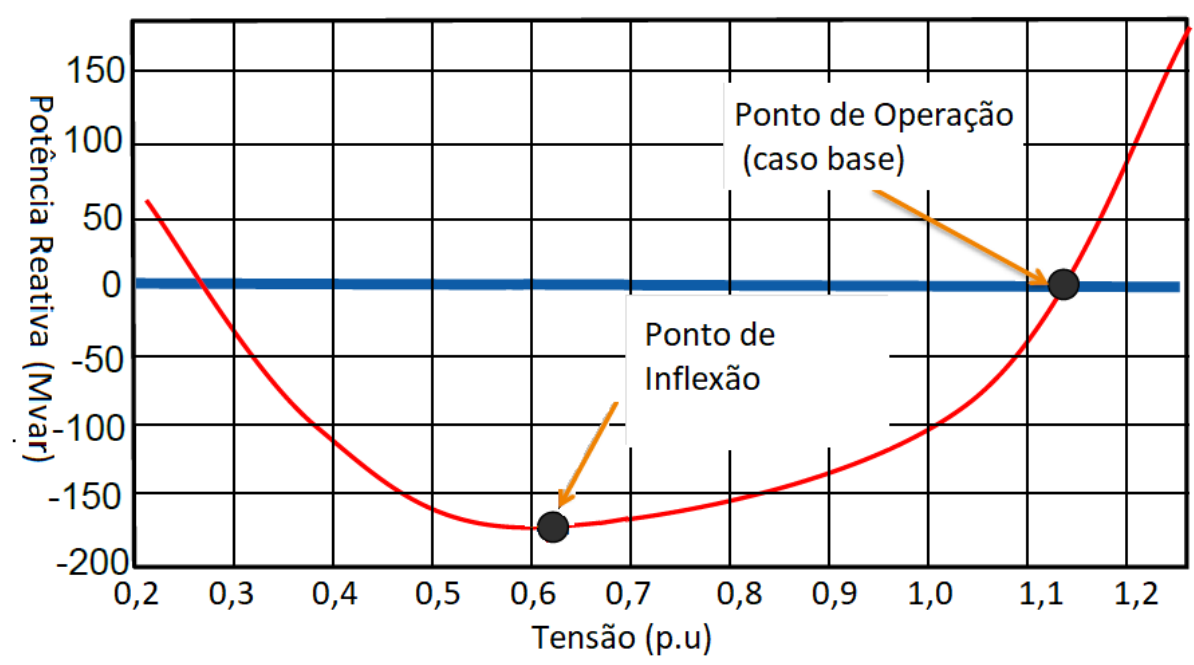

Figura 4.1: Curva QV[11]

A sequência do procedimento para obtenção da curva QV é: 
1. Executar o algoritmo do fluxo de potência para o caso base;

2. Escolher uma barra i para calcular a curva QV;

3. Modelar a barra escolhida como tendo um gerador síncrono fictício (barra do tipo PV sem limite de potência reativa);

4. Variar a tensão da barra i com um pequeno passo em torno da tensão do caso base;

5. Executar o algoritmo do fluxo de potência para o novo caso;

6. Anotar a potência reativa Q obtida pelo fluxo de potência e;

7. Repetir os passos 4 a 6 até que seja possível traçar a curva QV. A amplitude de variação da tensão $\mathrm{V}$ fica em torno de 0,9 a 1,1 p.u, valores de tensão fora dessa faixa não são práticos [12].

A curva QV não foi desenvolvida, em princípio, para estudar o problema da estabilidade de tensão. Sua origem advém da necessidade de solucionar problemas de fluxo de carga de difícil convergência [13].

Sabendo-se em qual barra ocorrem os problemas de convergência do fluxo de potência, acopla-se um compensador síncrono fictício nessa barra e arbitra-se uma tensão V'. Após executar o fluxo de potência com a tensão V' pré-estabelecida e com o compensador síncrono fictício acoplado, obtém-se uma potência reativa (fictícia) Q'. Desse ponto em diante, o procedimento consiste em reduzir vagarosamente o valor de tensão e, consequentemente, a potência reativa gerada pelo compensador fictício também é reduzida.

Finalmente, a tensão na barra atinge valores que não demandam potência reativa do gerador fictício e, portanto, está resolvido o problema do fluxo de carga.

As curvas 1 e 2, apresentadas na Figura 4.2, representam o caso descrito nos dois parágrafos anteriores. A interseção com o eixo Q igual a zero corresponde ao ponto sem necessidade de geração do compensador fictício $(\mathrm{Q}=0)$.

Q1 e Q2 são as margens de potência reativa, representando a distância entre o ponto de potência reativa mínima e o ponto sem compensação de potência reativa $(\mathrm{Q}=0)$.

Se a curva QV não corta o eixo horizontal $Q=0$, então o problema de fluxo de carga não teria solução (curva 3 da Figura 4.2). Nesse caso, a presença do compensador fictício de potência reativa sem limites de geração e absorção não é suficiente para solucionar o problema de fluxo de carga de difícil solução. 
Seria necessária a instalação de compensação de potência reativa real de um valor de, no mínimo, $Q_{3}$.

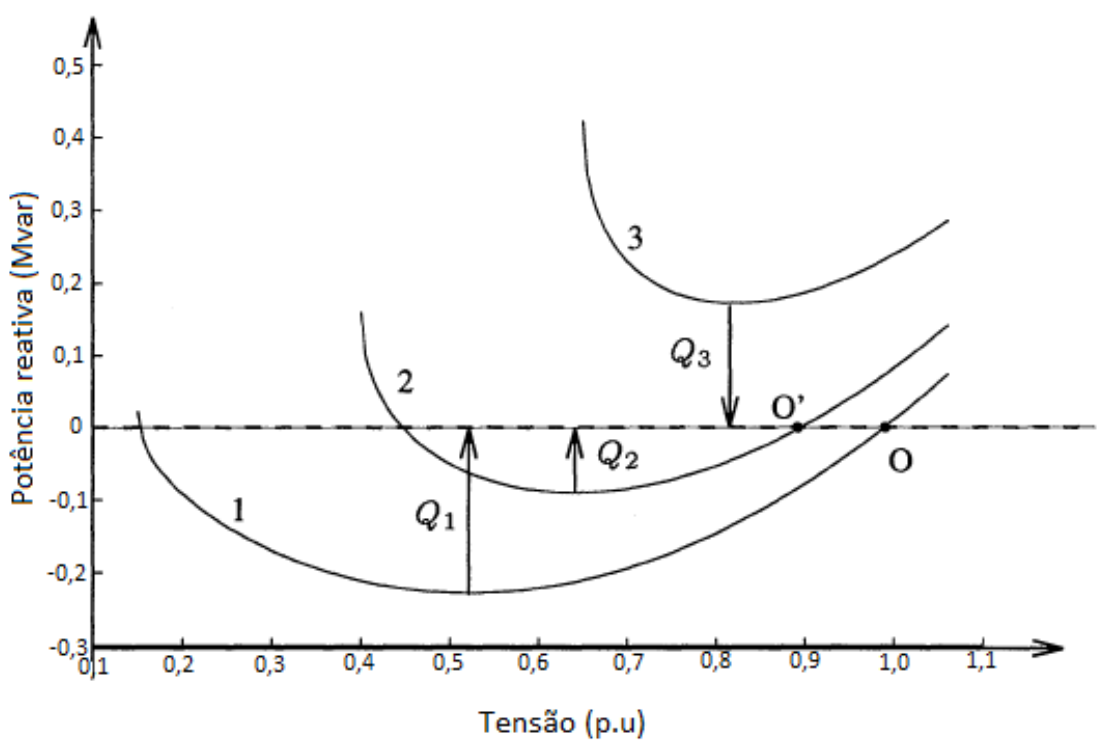

Figura 4.2: Curvas QV [13]

Por conseguinte, a aplicabilidade original da curva QV é solucionar problemas de fluxo de carga com díficil convergência. Posteriormente, a curva QV se mostrou interessante para avaliação da estabilidade de tensão.

Atualmente diversos programas podem ser utilizados para fornecimento da curva QV. O programa ANAREDE do CEPEL [14] foi utilizado para obtenção da curva QV nesse estudo.

A curva QV fornece informações que podem ser utilizadas para o estudo da estabilidade de tensão que serão apresentadas na Seção 4.3 .

\section{3}

\section{Avaliação da Estabilidade de Tensão com a Curva QV}

Tradicionalmente os equipamentos para controle de tensão nas barras do sistema (tap do transformador, reguladores automáticos de tensão) são construídos para operar satisfatoriamente na região em que o aumento de potência reativa $\mathrm{Q}$ é acompanhado por um aumento de tensão V [2]. Essa é considerada a região de operação usual do sistema. 
A curva QV é uma ferramenta de estudo de sistemas de potência que permite identificar e avaliar quando a tensão $\mathrm{V}$ e potência reativa $\mathrm{Q}$ são diretamente ou inversamente proporcionais. Sendo que o lado direito da curva QV corresponde à região usual de operação, com tensão e potência reativa diretamente proporcionais. O lado esquerdo da curva corresponde à região não usual de operação, com tensão e potência reativa inversamente proporcionais.

Por conseguinte, identificar em qual região o sistema de potência opera é fundamental para ações de controle eficientes.

A tensão e a potência reativa injetada são as condições iniciais que caracterizam uma determinada curva QV, sendo definidas como potência reativa do caso base (Qcb) e tensão do caso base (Vcb). A potência reativa no caso base também pode ser chamada de potência reativa injetada (Qinj).

Outro ponto importante na análise da curva QV é o ponto de fronteira, caracterizado pela potência reativa mínima que ocorre antes do sistema passar a operar na região esquerda do gráfico, Figura 4.1. No ponto de fronteira da curva QV, define-se a potência reativa de inflexão (Qinf) e a tensão de inflexão (Vinf).

A margem da curva QV (MQV) é definida como a diferença entre a potência reativa no ponto de fronteira e a potência reativa injetada (caso base) em (4.1).

$$
M Q V=\left(Q_{i n f}\right)-\left(Q_{i n j}\right)
$$

A margem da curva QV avalia o quanto de potência reativa que precisa ser fornecido para atingir o límite de potência reativa em determinada barra, independente dos limites físicos do gerador. A margem da curva QV também fornece a distância em MW do ponto de máximo carregamento.

Portanto, quanto maior a margem da curva QV $(M Q V)$, maior a distância que a barra em análise encontra-se de atingir a região de operação não usual do sistema. O lado da curva QV é obtido avaliando-se a tensão no caso base e a tensão no ponto de fronteira. Caso Vcb seja maior que Vinf, o lado da curva QV encontra-se no lado direito. Já se Vcb for menor que Vinf, o lado da curva QV encontra-se do lado esquerdo.

O lado da curva QV é um indicativo importante no estudo de estabilidade de tensão com curva QV, pois permite identificar em qual região de operação encontra-se. Esse índice será utilizado nos estudos de casos para análise da curva QV. 


\section{4}

\section{Obtendo o Índice $\left(\frac{\partial Q}{\partial V}\right)^{\prime \prime}$}

Nessa seção será explicado como obter o índice $\left(\frac{\partial Q}{\partial V}\right)^{\prime}$ realizando-se a redução da matriz Jacobiano duas vezes frente a algumas premissas adotadas.

Conforme descrito anteriormente em (2.30) e (2.31), as injeções de potência ativa e reativa do sistema de potência mantem uma relação com os ângulos e módulos de tensão na respectiva barra em estudo (4.2), através da matriz Jacobiano do sistema linearizado das equações de fluxo de potência.

Evidenciando as linhas e colunas correspondentes à barra i em análise obtém-se a expansão de (4.2) em (4.3).

$$
\begin{gathered}
{\left[\begin{array}{l}
\Delta P \\
\Delta Q
\end{array}\right]=[J]\left[\begin{array}{c}
\Delta \Theta \\
\Delta V
\end{array}\right]} \\
{\left[\begin{array}{c}
\overline{\Delta P} \\
\overline{\Delta Q} \\
\Delta P_{i} \\
\Delta Q_{i}
\end{array}\right]=\left[\begin{array}{ll}
A & B \\
\hline C & D
\end{array}\right]\left[\begin{array}{c}
\overline{\Delta \Theta} \\
\Delta V \\
\Delta \Theta_{i} \\
\Delta V_{i}
\end{array}\right]}
\end{gathered}
$$

Definem-se os elementos de (4.3):

- $\overline{\Delta P}$ é igual a $\Delta P$ excluindo o elemento $\Delta P_{i}$;

- $\overline{\Delta Q}$ é igual a $\Delta Q$ excluindo o elemento $\Delta Q_{i}$;

- $\overline{\Delta \Theta}$ é igual a $\Delta \Theta$ excluindo o elemento $\Delta \Theta_{i}$;

- $\overline{\Delta V}$ é igual a $\Delta V$ excluindo o elemento $\Delta V_{i}$;

- $\Delta P_{i}, \Delta Q_{i}, \Delta \Theta_{i}$ e $\Delta V_{i}$ são os elementos da barra i em análise.

A submatrix D em (4.3) relaciona as potências ativa e reativa injetadas em determinada barra com seu ângulo e módulo de tensão. Pretende-se avaliar as variações infinitesimais de $\Delta P_{i}$ e $\Delta Q_{i}$ e seus efeitos em $\Delta \Theta_{i}$ e $\Delta V_{i}$. Essa relação determina a matrix $D^{\prime}$ (Seção 3.5.1).

As premissas para o cálculo da matriz $D^{\prime}$ consideram que a potências ativa e reativa fora da barra i são constantes e, portanto, $\overline{\Delta P}$ e $\overline{\Delta Q}$ são iguais a zero:

$$
\left[\begin{array}{c}
0 \\
0 \\
\Delta P_{i} \\
\Delta Q_{i}
\end{array}\right]=\left[\begin{array}{ll}
A & B \\
\hline C & D
\end{array}\right]\left[\begin{array}{c}
\overline{\Delta \Theta} \\
\overline{\Delta V} \\
\Delta \Theta_{i} \\
\Delta V_{i}
\end{array}\right]
$$

Para facilitar o entendimento da resolução de (4.4), as parcelas do lado direito da equação são substituídas por X e Y: 


$$
\left[\begin{array}{l}
\overline{\Delta P} \\
\overline{\Delta Q}
\end{array}\right]=\mathbf{X} \quad \text { e } \quad\left[\begin{array}{l}
\Delta P_{i} \\
\Delta Q_{i}
\end{array}\right]=\mathbf{Y}
$$

e as parcelas do lado esquerdo são substituídas por Z e W:

$$
\left[\begin{array}{l}
\overline{\Delta \Theta} \\
\overline{\Delta V}
\end{array}\right]=\mathbf{Z} \quad \text { e } \quad\left[\begin{array}{c}
\Delta \Theta_{i} \\
\Delta V_{i}
\end{array}\right]=\mathbf{W}
$$

Assim (4.4) fornece (4.5):

$$
\left\{\begin{array} { l } 
{ \mathbf { 0 } = \mathbf { A } \cdot \mathbf { Z } + \mathbf { B } \cdot \mathbf { W } } \\
{ \mathbf { Y } = \mathbf { C } \cdot \mathbf { Z } + \mathbf { D } \cdot \mathbf { W } }
\end{array} \Rightarrow \left\{\begin{array}{l}
-\mathbf{A}^{-1} \cdot \mathbf{B} \cdot \mathbf{W}=\mathbf{Z}(1) \\
\mathbf{Y}=\mathbf{C} \cdot \mathbf{Z}+\mathbf{D} \cdot \mathbf{W}(2)
\end{array}\right.\right.
$$

Para resolver (4.5), substitui-se Z dado na equação (1) na equação (2) de (4.5):

$$
\mathbf{Y}=-C \cdot \mathbf{A}^{-1} \cdot \mathbf{B} \cdot \mathbf{W}+\mathbf{D} \cdot \mathbf{W} \Rightarrow\left[-C \cdot \mathbf{A}^{-1} \cdot \mathbf{B}+\mathbf{D}\right] \cdot \mathbf{W}
$$

Obtendo-se a matriz D' em (4.6):

$$
\left[\begin{array}{c}
\Delta P_{i} \\
\Delta Q_{i}
\end{array}\right]=\underbrace{\left[-C \cdot \mathbf{A}^{-1} \cdot \mathbf{B}+\mathbf{D}\right]}_{\mathbf{D}^{\prime}}\left[\begin{array}{c}
\Delta \Theta_{i} \\
\Delta V_{i}
\end{array}\right]
$$

A equação (4.6) tem D' como a matriz de derivadas parciais de $\Theta \mathrm{e} \mathrm{V}$ levando em conta todo o sistema:

$$
D^{\prime}=\left[\begin{array}{ll}
\frac{\partial P_{i}{ }^{\prime}}{\partial \Theta_{i}} & \frac{\partial P_{i}{ }^{\prime}}{\partial V_{i}} \\
\frac{\partial Q_{i}}{\partial \Theta_{i}} & \frac{\partial Q_{i}}{\partial V_{i}}
\end{array}\right]
$$

Portanto:

$$
\left[\begin{array}{c}
\Delta P_{i} \\
\Delta Q_{i}
\end{array}\right]=\left[\begin{array}{ll}
\frac{\partial P_{i}{ }^{\prime}}{\partial \Theta_{i}} & \frac{\partial P_{i}{ }^{\prime}}{\partial V_{i}} \\
\frac{\partial Q_{i}{ }^{\prime}}{\partial \Theta_{i}} & \frac{\partial Q_{i}}{\partial V_{i}}
\end{array}\right]\left[\begin{array}{c}
\Delta \Theta_{i} \\
\Delta V_{i}
\end{array}\right]
$$

O índice de estabilidade de tensão que pretende-se obter a relação entre $\Delta Q_{i}$ e $\Delta V_{i}$ em (4.8). Por conseguinte, é explicitando o elemento $\frac{\partial Q_{i}{ }^{\prime}}{\partial V_{i}}$ da matriz D'. Para isso, o mesmo procedimento utilizado para obter a matriz D' será repetido em (4.8).

Adotando-se como premissa que $\Delta P_{i}=0$ :

$$
\left[\begin{array}{c}
0 \\
\Delta Q_{i}
\end{array}\right]=\left[\begin{array}{ll}
\frac{\partial P_{i}{ }^{\prime}}{\partial \Theta_{i}} & \frac{\partial P_{i}{ }^{\prime}}{\partial V_{i}} \\
\frac{\partial Q_{i}}{\partial \Theta_{i}} & \frac{\partial Q_{i}}{\partial V_{i}}
\end{array}\right]\left[\begin{array}{c}
\Delta \Theta_{i} \\
\Delta V_{i}
\end{array}\right]
$$

Desenvolvendo-se (4.9) obtém-se (4.10): 


$$
\left\{\begin{array} { l } 
{ 0 = \frac { \partial P _ { i } { } ^ { \prime } } { \partial \Theta _ { i } } \Delta \Theta _ { i } + \frac { \partial P _ { i } { } ^ { \prime } } { \partial V _ { i } } \Delta V _ { i } } \\
{ \Delta Q _ { i } = \frac { \partial Q _ { i } { } ^ { \prime } } { \partial \Theta _ { i } } \Delta \Theta _ { i } + \frac { \partial Q _ { i } { } ^ { \prime } } { \partial V _ { i } } \Delta V _ { i } }
\end{array} \Rightarrow \left\{\begin{array}{l}
\Delta Q_{i}=\frac{\partial Q_{i}{ }^{\prime}}{\partial \Theta_{i}} \Delta \Theta_{i}+\frac{\partial Q_{i}{ }^{\prime}}{\partial V_{i}} \Delta V_{i}(1) \\
\Delta \Theta_{i}=\frac{\partial P_{i}{ }^{\prime}}{\partial V_{i}} \Delta V_{i}\left(\frac{\partial P_{i}{ }^{\prime}}{\partial \Theta_{i}}\right)^{-1}
\end{array}\right.\right.
$$

Substituindo-se $\Delta \Theta_{i}$ em (1) de (4.10), obtém-se o índice $\left(\frac{\partial Q}{\partial V}\right)^{\prime \prime}$ :

$$
\Delta Q_{i}=\left[\frac{\partial Q_{i}{ }^{\prime}}{\partial V_{i}}-\frac{\partial P_{i}{ }^{\prime}}{\partial V_{i}} \frac{\partial Q_{i}{ }^{\prime}}{\partial \Theta_{i}}\left(\frac{\partial P_{i}}{\partial \Theta_{i}}\right)^{\prime}-1\right] \Delta V_{i} \Rightarrow \Delta Q_{i}=\left[\frac{\partial Q_{i}{ }^{\prime}}{\partial V_{i}}-\frac{\partial P_{i}{ }^{\prime}}{\partial V_{i}} \frac{\partial Q_{i}{ }^{\prime}}{\partial \Theta_{i}}\left(\frac{\partial P_{i}{ }^{\prime}}{\partial \Theta_{i}}\right)^{-1}\right] \Delta V_{i}
$$

Portanto, dividindo-se (4.11) por $\frac{\partial P_{i}{ }^{\prime}}{\partial \Theta_{i}}$ obtém-se:

$$
\Delta Q_{i}=\left(\frac{\partial Q_{i}}{\partial V_{i}}\right)^{\prime \prime} \Delta V_{i} \Rightarrow\left(\frac{\partial Q_{i}}{\partial V_{i}}\right)^{\prime \prime}=\left[\frac{\Delta Q_{i}}{\Delta V_{i}}\right]
$$

$\mathrm{Ou}$

$$
\left(\frac{\partial Q_{i}}{\partial V_{i}}\right)^{\prime \prime}=\frac{\operatorname{det}\left(D^{\prime}\right)}{\frac{\partial P_{i}^{\prime}}{\partial \Theta_{i}}}
$$

O índice $\left(\frac{\partial Q_{i}}{\partial V_{i}}\right)^{\prime \prime}$ apresentado em (4.12) é implementado computacionalmente nesse trabalho.

$\mathrm{Na}$ Seção 4.5 será apresentado as vantagens desse índice e sua aplicabilidade na avaliação do problema de estabilidade de tensão.

\section{5}

\section{Avaliação da Estabilidade de Tensão Utilizando o Índice $\left(\frac{\partial Q}{\partial V}\right)^{"}$}

O índice $\left(\frac{\partial Q}{\partial V}\right)$ " é a relação entre a potência reativa e a tensão em todos os barramentos do sistema $[2,10]$. O índice $\left(\frac{\partial Q}{\partial V}\right)$ " positivo indica que um aumento de tensão levaria a um aumento de potência reativa em determinado barramento, assim como uma diminuição de tensão levaria a uma diminuição da potência reativa. Esse comportamento referente ao índice $\left(\frac{\partial Q}{\partial V}\right)$ " caracteriza que a rede opera na região normal [2].

Por outro lado, valores negativos do índice $\left(\frac{\partial Q}{\partial V}\right)$ " são um indicativo de que o sistema opera na região anormal. Nessa situação aumentos de tensão seriam acompanhados pela diminuição da potência reativa [2].

\section{6}

\section{Incompatibilidade na Avaliação da Estabilidade de Tensão}

Essa seção tem como objetivo apresentar as diferenças e similaridades para obtenção do índice $\left(\frac{\partial Q}{\partial V}\right)$ " e a curva QV como ferramentas para avaliação da estabilidade de tensão. 
A principal diferença é que o cálculo do índice $\left(\frac{\partial Q}{\partial V}\right)$ " ocorre em determinado ponto de operação, enquanto a curva QV utiliza diversos pontos de operação.

O índice $\left(\frac{\partial Q}{\partial V}\right)$ " como a própria nomenclatura indica é a variação infinitesimal de duas grandezas, Q e V. Pode-se dizer que esse índice é a derivada de Q em relação a V. Já a margem obtida da curva QV utiliza diversos pontos de operação, sendo a relação das variações finitas em $\Delta V$ e $\Delta Q$. Portanto, a margem da curva $Q V$ representa uma variação de $\Delta V$ com $\Delta Q$, onde $\Delta V$ e $\Delta Q$ são variações de grande magnitude.

Resumindo, enquanto $\left(\frac{\partial Q}{\partial V}\right)$ " define variações infinitesimais (uma tangente), por outro lado, a curva QV expressa margens $(\Delta V$ e $\Delta Q)$ com a formação de um triângulo, Figura 4.1. Essa diferença matemática pode levar a uma avaliação de estabilidade de tensão com resultados diferentes.

Entretanto, a obtenção dos dois índices também apresenta similaridades. No cálculo do índice $\left(\frac{\partial Q}{\partial V}\right)$ " estuda-se na verdade uma variação infinitesimal entre Q e V, essa variação infinitesimal é absorvida pela barra swing no que diz respeito às variações das perdas ativas e as barras PV (com Q livre) absorvem as variações infinitesimais no $\mathrm{Q}$ e as variações das perdas reativas.

Já quando se faz a curva QV, varia-se V, resolve-se o fluxo de potência e calcula Q. Exatamente da mesma forma que no cálculo do índice $\left(\frac{\partial Q}{\partial V}\right)$ " quem absorve as variações das perdas ativas é a barra swing, e quem absorve a variação das perdas reativas são todas as barras do tipo PV, demostrando-se similaridade nesse aspecto.

Como avaliado no decorrer dessa seção, a maneira de obter os dois índices de estabilidade de tensão é diferente na maneira de ser calculada. Portanto, é possível que para determinados casos de operação o resultado da avaliação de estabilidade de tensão deva ser diferente entre os índices.

\section{7}

\section{Sumário do Capítulo}

Nesse capítulo foram apresentados dois métodos para avaliação da estabilidade de tensão.

O método da curva QV realiza o fluxo da potência para calcular a margem da potência reativa e o lado da curva QV em determina barra do sistema. Enquanto o índice de sensibilidade $\left(\frac{\partial Q}{\partial V}\right)$ " cálcula a derivada de Q em relação a $\mathrm{V}$ em um único ponto de operação.

A avaliação do estado de operação do sistema segundo a estabilidade de tensão pode divergir quando comparados os resultados desses dois índices. Um 
estudo de caso para avaliar e comparar possíveis divergências nos dois índices é apresentado no Capítulo 5 . 


\section{5}

\section{Casos de Estudo}

\section{1}

\section{Introdução}

O objetivo deste capítulo é abordar possíveis inconsistências e incoerências na avaliação da estabilidade de tensão ao utilizar o índice $\left(\frac{\partial Q}{\partial V}\right)^{\prime}$ e a margem da curva QV em sistema de potência próximos de seu carregamento máximo, conforme abordado na Seção 4.6.

A margem e o lado da curva QV são obtidos através do programa ANAREDE, ferramenta computacional utilizada para análise de sistemas elétricos de potência em regime permanente desenvolvida pelo Cepel.

O índice $\left(\frac{\partial Q}{\partial V}\right)$ " é calculado utilizando-se a matriz Jacobiana em determinado ponto de operação. Uma ferramenta computacional foi programada em Matlab para calcular o índice $\left(\frac{\partial Q}{\partial V}\right)$ ".

O procedimento adotado no caso estudado tem como referência o relatório produzido para o Cepel pela PUC-Rio [15]. Esse estudo consiste em aumentar o patamar de carga utilizando o Fluxo de Potência Continuado (FPC), obtendo-se vários pontos de operação e neles calculando-se os índices de interesse.

Nesse capítulo é desenvolvido um estudo comparativo com o índice $\left(\frac{\partial Q}{\partial V}\right)$ e a margem da curva QV na Seção 5.2. E na Seção 5.3 é apresentado um estudo avaliando-se a incongruência dos resultados da margem de potência reativa da curva QV.

\section{2}

\section{Estudo Comparativo do Índice $\left(\frac{\partial Q}{\partial V}\right)$ " e Margem da Curva QV}

O estudo desenvolvido nessa Seção realizou-se com o sistema teste IEEE 14 barras [16], com o objetivo de avaliar as possíveis divergências nos índices utilizados. Aumenta-se gradualmente a carga do sistema teste, até atingir a região de máximo carregamento e, simultaneamente, em cada patamar de carga é calculado o índice $\left(\frac{\partial Q}{\partial V}\right)$ " e a margem da curva $Q V$.

O sistema IEEE 14 barras representa uma fração do sistema de potência dos EUA em fevereiro de 1962. Esse sistema consiste em um modelo bastante 
testado no meio acadêmico e que frequentemente é usado por pesquisadores e engenheiros na implementação de novas ferramentas e conceitos na área de sistemas de potência.

Em resumo, o sistema possui 2 centrais de geração e 3 compensadores síncronos. Das 14 barras do sistema, 11 possuem carga. Na Figura 5.1 apresenta-se o sistema IEEE 14 barras, enquanto os seus dados podem ser encontrados no Apêndice A.

(G) Geradores
(C) ${ }_{\text {Síncronos }}$

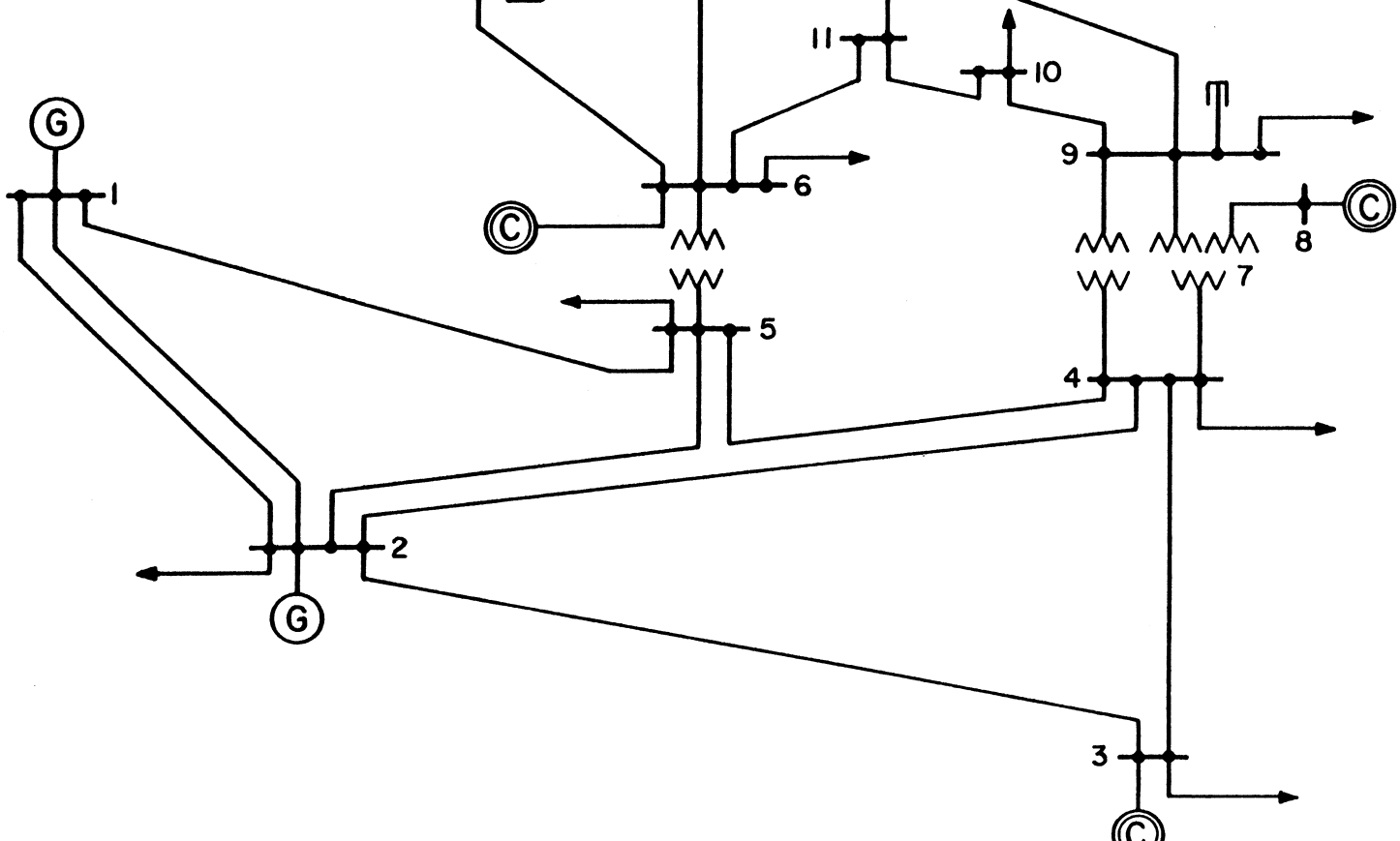

Figura 5.1: Diagrama Unifilar do Sistema IEEE 14 Barras [16]

\subsection{1}

\section{Estudos com Crescimento de Carga e Geração em uma Única Barra}

Para o sistema teste IEEE 14 barras é definido um ponto de operação inicial e, partindo desse ponto, são realizados aumentos sucessivos de carga na barra 2 com fator de potência constante.

A potência ativa necessária para acompanhar o aumento de carga é fornecida pela barra 1 (barra de referência do sistema). 
A simulação do FPC é realizada no ANAREDE com os seguintes parâmetros ativados: NEWT, QLIM, VLIM, CREM, CTAP, RCVG, FILE, RBAR e BPSI. A opção de fluxo de potência continuado parametrizada é ativada. Para esse estudo utilizou-se a versão 11.2.4.

A simulação fornece [140] pontos de operação, cada qual com um diferente patamar de carga. Cada ponto de operação é obtido com um aumento de carga de $20 \%$ com relação ao ponto anterior.

Os 140 casos ou pontos de operação possuem fluxo de carga convergido no ANAREDE. Porém, o algoritmo de fluxo de potência do Matlab não é capaz de convergir todos os casos para o cálculo do índice $\left(\frac{\partial Q}{\partial V}\right)$ ". Assim, alguns casos serão retirados da análise.

O comando DGER no ANAREDE é habilitado para configurar a barra 1 como responsável por fornecer potência ativa para o sistema, frente ao aumento de carga. A barra 1 é barra swing do sistema, responsável por absorver as perdas de potência ativa.

Assim, a circulação de potência ativa do sistema restringe-se principalmente entre as barras 1 e 2 .

São apresentados tensão, potência reativa e ativa, índice de sensibilidade $\left(\frac{\partial Q}{\partial V}\right)$ " e margem da curva QV para as barras 1, 2, 4 e 5 nas próximas seções.

A margem da curva QV denotada por $\left(M_{Q V}\right)$ nas Tabelas 5.1, 5.3, $5.4 \mathrm{e}$ 5.5 tem valor positivo quando o ponto de operação se encontra no lado direito da curva QV e valor negativo, caso o ponto de operação esteja no lado esquerdo da curva. E conforme definido na Seção 4.3, a margem da curva QV informa a distância em MW do ponto de máximo carregamento.

\subsubsection{1}

\section{Análise da Barra 1}

A análise da barra 1 tem como objetivo mostrar o comportamento do índice $\left(\frac{\partial Q}{\partial V}\right)$ ", a margem e o lado da curva QV após sucessivos aumentos no patamar de carga na barra 2 .

Na Tabela 5.1 apresentam-se tipo da barra, potência ativa e reativa gerada, tensão, ângulo, e aumento percentual da carga. O índice $\left(\frac{\partial Q}{\partial V}\right)$ " e margem da curva QV $\left(M_{Q V}\right)$ também são apresentados na Tabela 5.1.

A barra 1 é a barra de referência do sistema, portanto $V$ e $\Theta$ são constantes nessa barra. Essa barra absorve as perdas de potência ativa e, junto com as barras PV, as perdas reativa do sistema.

O Caso 1 corresponde ao caso-base do sistema e possui potência ativa na barra 1 igual a 232,4 MW e tensão de 1,06 p.u. A $M_{Q V}$ não é calculada pelo programa ANAREDE no Caso 1, na barra 1. 
O índice $\left(\frac{\partial Q}{\partial V}\right)$ " apresenta descontinuidade quando ocorre perda de controle de tensão, ou seja, quando uma determinada barra atinge o seu limite de geração de potência reativa.

O fenômeno da perda de controle de tensão ocorre no Caso 3 para a barra 2, Caso 16 para a barra 3, Caso 27 para barra 6 e Caso 28 para a barra 8 , como observado na Tabela 5.2.

Na Tabela 5.2 apresenta-se, para as barras 1 a 8, o tipo de barra, permitindo observar a perda de controle de tensão que ocorre nas barras do tipo PV.

A Tabela 5.2 segue a referência do programa ANAREDE [14], onde:

1. Barra do tipo 2 é barra $V \Theta$, de referência ou swing.

2. Barras do tipo 1 é barra PQ.

3. Barras do tipo 0 é barra PV.

4. Barras do tipo -1 é barra PQ que perderam o controle de tensão.

Tabela 5.1: Análise da Barra 1 - Crescimento de Carga na Barra 2

\begin{tabular}{ccccccccc}
\hline Casos & Tipo & $\begin{array}{c}\mathbf{P G}_{1} \\
(\mathbf{M W})\end{array}$ & $\begin{array}{c}\mathbf{Q G}_{1} \\
(\mathbf{M v a r})\end{array}$ & $\begin{array}{c}\mathbf{V}_{1} \\
(\mathbf{p . u} .)\end{array}$ & $\begin{array}{c}\Theta_{1} \\
\left({ }^{\circ}\right)\end{array}$ & $\begin{array}{c}\Delta \\
\text { Carga (\%) }\end{array}$ & $M_{Q V}{ }^{1}$ & $\left(\frac{\partial Q}{\partial V}\right)^{\prime \prime}$ \\
\hline 1 & 2,0 & 232,4 & $-16,5$ & 1,06 & 0,0 & 0,0 & NaN & 21,290 \\
\hline 2 & 2,0 & 237,0 & $-17,5$ & 1,06 & $-0,0$ & 20,0 & 27,5 & 21,271 \\
\hline 3 & 2,0 & 241,6 & $-17,1$ & 1,06 & $-0,0$ & 40,0 & 24,8 & 8,403 \\
\hline 4 & 2,0 & 246,2 & $-15,3$ & 1,06 & $-0,0$ & 60,0 & 23,3 & 8,393 \\
\hline 5 & 2,0 & 250,8 & $-13,4$ & 1,06 & $-0,0$ & 80,0 & 21,5 & 8,383 \\
\hline 6 & 2,0 & 255,4 & $-11,6$ & 1,06 & $-0,0$ & 100,0 & 19,8 & 8,372 \\
\hline 7 & 2,0 & 260,0 & $-9,7$ & 1,06 & $-0,0$ & 120,0 & 18,8 & 8,362 \\
\hline 8 & 2,0 & 264,6 & $-7,8$ & 1,06 & $-0,0$ & 140,0 & 17,6 & 8,350 \\
\hline 9 & 2,0 & 269,2 & $-5,9$ & 1,06 & $-0,0$ & 160,0 & 16,3 & 8,339 \\
\hline 10 & 2,0 & 273,9 & $-4,0$ & 1,06 & $-0,0$ & 180,0 & 14,8 & 8,327 \\
\hline 11 & 2,0 & 278,5 & $-2,0$ & 1,06 & $-0,0$ & 200,0 & 12,9 & 8,315 \\
\hline 12 & 2,0 & 283,2 & $-0,0$ & 1,06 & $-0,0$ & 220,0 & 11,0 & 8,302 \\
\hline 13 & 2,0 & 287,9 & 2,0 & 1,06 & $-0,0$ & 240,0 & 10,1 & 8,289 \\
\hline 14 & 2,0 & 292,5 & 4,0 & 1,06 & $-0,0$ & 260,0 & 8,9 & 8,276 \\
\hline 15 & 2,0 & 297,2 & 6,0 & 1,06 & $-0,0$ & 280,0 & 7,6 & 8,262 \\
\hline 16 & 2,0 & 301,9 & 8,3 & 1,06 & $-0,0$ & 300,0 & 6,3 & 4,073 \\
\hline 17 & 2,0 & 306,6 & 11,2 & 1,06 & $-0,0$ & 320,0 & 5,2 & 4,054 \\
\hline 18 & 2,0 & 311,3 & 14,1 & 1,06 & $-0,0$ & 340,0 & 4,1 & 4,034 \\
\hline 19 & 2,0 & 316,1 & 17,1 & 1,06 & $-0,0$ & 360,0 & 3,9 & 4,013 \\
\hline 20 & 2,0 & 320,8 & 20,1 & 1,06 & $-0,0$ & 380,0 & 3,7 & 3,991 \\
\hline 21 & 2,0 & 325,5 & 23,1 & 1,06 & $-0,0$ & 400,0 & 3,2 & 3,970 \\
\hline & & & & & & & &
\end{tabular}




\begin{tabular}{|c|c|c|c|c|c|c|c|c|}
\hline Casos & Tipo & $\begin{array}{c}\mathbf{P G}_{1} \\
(\mathrm{MW})\end{array}$ & $\begin{array}{c}\mathbf{Q G}_{1} \\
\text { (Mvar) }\end{array}$ & $\begin{array}{c}\mathrm{V}_{1} \\
\text { (p.u.) }\end{array}$ & $\begin{array}{l}\Theta_{1} \\
\left(^{\circ}\right)\end{array}$ & $\begin{array}{c}\Delta \\
\text { Carga }(\%)\end{array}$ & $M_{Q V}$ & $\left(\frac{\partial Q}{\partial V}\right)^{\prime \prime}$ \\
\hline 22 & 2,0 & 330,3 & 26,1 & 1,06 & $-0,0$ & 420,0 & 2,4 & 3,947 \\
\hline 23 & 2,0 & 335,1 & 29,2 & 1,06 & $-0,0$ & 440,0 & 1,3 & 3,924 \\
\hline 24 & 2,0 & 339,8 & 32,3 & 1,06 & $-0,0$ & 460,0 & 0,1 & 3,900 \\
\hline 25 & 2,0 & 344,6 & 35,4 & 1,06 & $-0,0$ & 480,0 & 0,0 & 3,875 \\
\hline 26 & 2,0 & 349,4 & 38,6 & 1,06 & $-0,0$ & 500,0 & 0,0 & 3,850 \\
\hline 27 & 2,0 & 354,2 & 42,3 & 1,06 & $-0,0$ & 520,0 & 0,0 & 0,712 \\
\hline 28 & 2,0 & 359,1 & 46,4 & 1,06 & $-0,0$ & 540,0 & 0,0 & $-2,945$ \\
\hline 29 & 2,0 & 363,9 & 50,8 & 1,06 & $-0,0$ & 560,0 & 0,0 & $-2,995$ \\
\hline 30 & 2,0 & 368,8 & 55,3 & 1,06 & $-0,0$ & 580,0 & $-0,0$ & $-3,047$ \\
\hline 31 & 2,0 & 373,7 & 59,8 & 1,06 & $-0,0$ & 600,0 & $-1,2$ & $-3,100$ \\
\hline 32 & 2,0 & 378,6 & 64,3 & 1,06 & $-0,0$ & 620,0 & $-2,5$ & $-3,155$ \\
\hline 33 & 2,0 & 383,5 & 68,9 & 1,06 & $-0,0$ & 640,0 & $-3,2$ & $-3,213$ \\
\hline 34 & 2,0 & 388,4 & 73,6 & 1,06 & $-0,0$ & 660,0 & $-3,5$ & $-3,271$ \\
\hline 35 & 2,0 & 393,3 & 78,3 & 1,06 & $-0,0$ & 680,0 & $-3,5$ & $-3,333$ \\
\hline 36 & 2,0 & 398,3 & 83,0 & 1,06 & $-0,0$ & 700,0 & $-3,9$ & $-3,394$ \\
\hline 37 & 2,0 & 403,3 & 87,8 & 1,06 & $-0,0$ & 720,0 & $-5,2$ & $-3,458$ \\
\hline 38 & 2,0 & 408,2 & 92,7 & 1,06 & $-0,0$ & 740,0 & $-6,6$ & $-3,526$ \\
\hline 39 & 2,0 & 413,3 & 97,6 & 1,06 & $-0,0$ & 760,0 & $-7,5$ & $-3,593$ \\
\hline 40 & 2,0 & 418,3 & 102,5 & 1,06 & $-0,0$ & 780,0 & $-7,7$ & $-3,665$ \\
\hline 41 & 2,0 & 423,3 & 107,6 & 1,06 & $-0,0$ & 800,0 & $-7,9$ & $-3,738$ \\
\hline 42 & 2,0 & 428,4 & 112,7 & 1,06 & $-0,0$ & 820,0 & $-8,7$ & $-3,812$ \\
\hline 43 & 2,0 & 433,4 & 117,8 & 1,06 & $-0,0$ & 840,0 & $-10,3$ & $-3,891$ \\
\hline 44 & 2,0 & 438,5 & 123,0 & 1,06 & $-0,0$ & 860,0 & $-11,8$ & $-3,971$ \\
\hline 45 & 2,0 & 443,6 & 128,3 & 1,06 & $-0,0$ & 880,0 & $-12,6$ & $-4,055$ \\
\hline 46 & 2,0 & 448,8 & 133,6 & 1,06 & $-0,0$ & 900,0 & $-12,9$ & $-4,140$ \\
\hline 47 & 2,0 & 453,9 & 139,0 & 1,06 & $-0,0$ & 920,0 & $-13,2$ & $-4,227$ \\
\hline 48 & 2,0 & 459,1 & 144,5 & 1,06 & $-0,0$ & 940,0 & $-14,8$ & $-4,320$ \\
\hline 49 & 2,0 & 464,3 & 150,0 & 1,06 & $-0,0$ & 960,0 & $-16,5$ & $-4,414$ \\
\hline 50 & 2,0 & 469,5 & 155,6 & 1,06 & $-0,0$ & 980,0 & $-18,3$ & $-4,514$ \\
\hline 51 & 2,0 & 474,7 & 161,3 & 1,06 & $-0,0$ & 1000,0 & $-19,0$ & $-4,614$ \\
\hline 52 & 2,0 & 480,0 & 167,1 & 1,06 & $-0,0$ & 1020,0 & $-19,5$ & $-4,717$ \\
\hline 53 & 2,0 & 485,3 & 172,9 & 1,06 & $-0,0$ & 1040,0 & $-20,5$ & $-4,828$ \\
\hline 54 & 2,0 & 490,6 & 178,8 & 1,06 & $-0,0$ & 1060,0 & $-22,4$ & $-4,939$ \\
\hline 55 & 2,0 & 495,9 & 184,8 & 1,06 & $-0,0$ & 1080,0 & $-24,5$ & $-5,058$ \\
\hline 56 & 2,0 & 501,3 & 190,9 & 1,06 & $-0,0$ & 1100,0 & $-26,3$ & $-5,177$ \\
\hline 57 & 2,0 & 506,7 & 197,1 & 1,06 & $-0,0$ & 1120,0 & $-27,0$ & $-5,301$ \\
\hline 58 & 2,0 & 512,1 & 203,4 & 1,06 & $-0,0$ & 1140,0 & $-27,6$ & $-5,433$ \\
\hline 59 & 2,0 & 517,5 & 209,7 & 1,06 & $-0,0$ & 1160,0 & $-29,8$ & $-5,567$ \\
\hline 60 & 2,0 & 523,0 & 216,2 & 1,06 & $-0,0$ & 1180,0 & $-32,1$ & $-5,709$ \\
\hline 61 & 2,0 & 528,5 & 222,7 & 1,06 & $-0,0$ & 1200,0 & $-34,4$ & $-5,853$ \\
\hline 62 & 2,0 & 534,0 & 229,4 & 1,06 & $-0,0$ & 1220,0 & $-36,0$ & $-6,003$ \\
\hline 63 & 2,0 & 539,6 & 236,2 & 1,06 & $-0,0$ & 1240,0 & $-37,0$ & $-6,164$ \\
\hline 64 & 2,0 & 545,2 & 243,0 & 1,06 & $-0,0$ & 1260,0 & $-38,8$ & $-6,326$ \\
\hline
\end{tabular}




\begin{tabular}{|c|c|c|c|c|c|c|c|c|}
\hline Casos & Tipo & $\begin{array}{c}\mathbf{P G}_{1} \\
(\mathrm{MW})\end{array}$ & $\begin{array}{c}\mathrm{QG}_{1} \\
\text { (Mvar) }\end{array}$ & $\begin{array}{c}\mathrm{V}_{1} \\
\text { (p.u.) }\end{array}$ & $\begin{array}{l}\Theta_{1} \\
\left(^{\circ}\right)\end{array}$ & $\begin{array}{c}\Delta \\
\text { Carga }(\%)\end{array}$ & $M_{Q V}$ & $\left(\frac{\partial Q}{\partial V}\right)^{\prime \prime}$ \\
\hline 65 & 2,0 & 550,8 & 250,0 & 1,06 & $-0,0$ & 1280,0 & $-41,5$ & $-6,501$ \\
\hline 66 & 2,0 & 556,4 & 257,1 & 1,06 & $-0,0$ & 1300,0 & $-44,3$ & $-6,677$ \\
\hline 67 & 2,0 & 562,2 & 264,4 & 1,06 & $-0,0$ & 1320,0 & $-47,0$ & $-6,862$ \\
\hline 68 & 2,0 & 567,9 & 271,8 & 1,06 & $-0,0$ & 1340,0 & $-48,4$ & $-7,060$ \\
\hline 69 & 2,0 & 573,7 & 279,3 & 1,06 & $-0,0$ & 1360,0 & $-50,1$ & $-7,261$ \\
\hline 70 & 2,0 & 579,5 & 286,9 & 1,06 & $-0,0$ & 1380,0 & $-53,3$ & $-7,479$ \\
\hline 71 & 2,0 & 585,4 & 294,7 & 1,06 & $-0,0$ & 1400,0 & $-56,6$ & $-7,700$ \\
\hline 72 & 2,0 & 591,3 & 302,7 & 1,06 & $-0,0$ & 1420,0 & $-59,8$ & $-7,932$ \\
\hline 73 & 2,0 & 597,2 & 310,8 & 1,06 & $-0,0$ & 1440,0 & $-62,5$ & $-8,183$ \\
\hline 74 & 2,0 & 603,3 & 319,1 & 1,06 & $-0,0$ & 1460,0 & $-64,5$ & $-8,439$ \\
\hline 75 & 2,0 & 609,3 & 327,6 & 1,06 & $-0,0$ & 1480,0 & $-68,2$ & $-8,717$ \\
\hline 76 & 2,0 & 615,5 & 336,2 & 1,06 & $-0,0$ & 1500,0 & $-72,1$ & $-9,002$ \\
\hline 77 & 2,0 & 621,6 & 345,1 & 1,06 & $-0,0$ & 1520,0 & $-76,1$ & $-9,303$ \\
\hline 78 & 2,0 & 627,9 & 354,2 & 1,06 & $-0,0$ & 1540,0 & $-80,2$ & $-9,632$ \\
\hline 79 & 2,0 & 634,2 & 363,5 & 1,06 & $-0,0$ & 1560,0 & $-82,9$ & $-9,970$ \\
\hline 80 & 2,0 & 640,6 & 373,0 & 1,06 & $-0,0$ & 1580,0 & $-87,1$ & $-10,341$ \\
\hline 81 & 2,0 & 647,1 & 382,8 & 1,06 & $-0,0$ & 1600,0 & $-92,1$ & $-10,724$ \\
\hline 82 & 2,0 & 653,6 & 392,9 & 1,06 & $-0,0$ & 1620,0 & $-97,1$ & $-11,134$ \\
\hline 83 & 2,0 & 660,3 & 403,3 & 1,06 & $-0,0$ & 1640,0 & $-102,3$ & $-11,585$ \\
\hline 84 & 2,0 & 667,0 & 414,0 & 1,06 & $-0,0$ & 1660,0 & $-106,8$ & $-12,057$ \\
\hline 85 & 2,0 & 673,8 & 425,1 & 1,06 & $-0,0$ & 1680,0 & $-112,0$ & $-12,581$ \\
\hline 86 & 2,0 & 680,8 & 436,6 & 1,06 & $-0,0$ & 1700,0 & $-118,4$ & $-13,132$ \\
\hline 87 & 2,0 & 687,9 & 448,4 & 1,06 & $-0,0$ & 1720,0 & $-125,0$ & $-13,731$ \\
\hline 88 & 2,0 & 695,1 & 460,8 & 1,06 & $-0,0$ & 1740,0 & $-131,9$ & $-14,406$ \\
\hline 89 & 2,0 & 702,5 & 473,6 & 1,06 & $-0,0$ & 1760,0 & $-138,8$ & $-15,126$ \\
\hline 90 & 2,0 & 710,0 & 487,1 & 1,06 & $-0,0$ & 1780,0 & $-146,0$ & $-15,948$ \\
\hline 91 & 2,0 & 717,7 & 501,2 & 1,06 & $-0,0$ & 1800,0 & $-154,8$ & $-16,838$ \\
\hline 92 & 2,0 & 725,7 & 516,1 & 1,06 & $-0,0$ & 1820,0 & $-164,3$ & $-17,839$ \\
\hline 93 & 2,0 & 733,9 & 531,8 & 1,06 & $-0,0$ & 1840,0 & $-174,4$ & $-19,015$ \\
\hline 94 & 2,0 & 742,4 & 548,6 & 1,06 & $-0,0$ & 1860,0 & $-185,4$ & $-20,334$ \\
\hline 95 & 2,0 & 751,3 & 566,7 & 1,06 & $-0,0$ & 1880,0 & $-196,9$ & $-21,933$ \\
\hline 96 & 2,0 & 760,7 & 586,4 & 1,06 & $-0,0$ & 1900,0 & $-211,1$ & $-23,803$ \\
\hline 97 & 2,0 & 770,7 & 608,2 & 1,06 & $-0,0$ & 1920,0 & $-227,3$ & $-26,125$ \\
\hline 98 & 2,0 & 781,5 & 633,0 & 1,06 & $-0,0$ & 1940,0 & $-246,1$ & $-29,250$ \\
\hline 99 & 2,0 & 793,7 & 662,3 & 1,06 & $-0,0$ & 1960,0 & $-269,5$ & $-33,541$ \\
\hline 100 & 2,0 & 808,4 & 700,2 & 1,06 & $-0,0$ & 1980,0 & $-373,8$ & $-40,912$ \\
\hline 101 & 2,0 & 834,8 & 778,8 & 1,06 & $-0,0$ & 2000,0 & $-378,4$ & $-67,093$ \\
\hline 102 & 2,0 & 836,2 & 783,5 & 1,06 & $-0,0$ & 2000,0 & 0,0 & $-69,728$ \\
\hline 103 & 2,0 & 838,4 & 791,2 & 1,06 & $-0,0$ & 2001,0 & 0,0 & 44,069 \\
\hline 104 & 2,0 & 840,6 & 798,8 & 1,06 & $-0,0$ & 2000,0 & 0,0 & $-80,455$ \\
\hline 105 & 2,0 & 842,7 & 806,3 & 1,06 & $-0,0$ & 2000,0 & 0,0 & $-84,133$ \\
\hline 106 & 2,0 & 844,7 & 813,8 & 1,06 & $-0,0$ & 1999,0 & 0,0 & $-92,071$ \\
\hline 107 & 2,0 & 846,7 & 821,1 & 1,06 & $-0,0$ & 1998,0 & 0,0 & $-98,439$ \\
\hline
\end{tabular}




\begin{tabular}{|c|c|c|c|c|c|c|c|c|}
\hline Casos & Tipo & $\begin{array}{c}\mathbf{P G}_{1} \\
(\mathrm{MW})\end{array}$ & $\begin{array}{c}\mathbf{Q G}_{1} \\
\text { (Mvar) }\end{array}$ & $\begin{array}{c}\mathrm{V}_{1} \\
\text { (p.u.) }\end{array}$ & $\begin{array}{l}\Theta_{1} \\
\left(^{\circ}\right)\end{array}$ & $\begin{array}{c}\Delta \\
\text { Carga (\%) }\end{array}$ & $M_{Q V}$ & $\left(\frac{\partial Q}{\partial V}\right)^{\prime \prime}$ \\
\hline 108 & 2,0 & 848,5 & 828,4 & 1,06 & $-0,0$ & 1997,0 & 0,0 & $-106,327$ \\
\hline 109 & 2,0 & 850,3 & 835,6 & 1,06 & $-0,0$ & 1996,0 & 0,0 & $-115,602$ \\
\hline 110 & 2,0 & 852,0 & 842,6 & 1,06 & $-0,0$ & 1994,0 & 0,0 & $-126,617$ \\
\hline 111 & 2,0 & 853,6 & 849,6 & 1,06 & $-0,0$ & 1992,0 & 0,0 & $-138,024$ \\
\hline 112 & 2,0 & 855,2 & 856,4 & 1,06 & $-0,0$ & 1990,0 & 0,0 & $-154,438$ \\
\hline 113 & 2,0 & 856,6 & 863,2 & 1,06 & $-0,0$ & 1987,0 & 0,0 & $-175,006$ \\
\hline 114 & 2,0 & 858,0 & 869,8 & 1,06 & $-0,0$ & 1985,0 & 0,0 & $-199,235$ \\
\hline 115 & 2,0 & 859,3 & 876,3 & 1,06 & $-0,0$ & 1982,0 & 0,0 & $-231,018$ \\
\hline 116 & 2,0 & 860,6 & 882,7 & 1,06 & $-0,0$ & 1979,0 & 0,0 & $-271,474$ \\
\hline 117 & 2,0 & 861,7 & 889,0 & 1,06 & $-0,0$ & 1975,0 & 0,0 & $-336,412$ \\
\hline 118 & 2,0 & 862,8 & 895,2 & 1,06 & $-0,0$ & 1972,0 & 0,0 & $-434,130$ \\
\hline 119 & 2,0 & 863,8 & 901,3 & 1,06 & $-0,0$ & 1968,0 & 0,0 & $-614,829$ \\
\hline 120 & 2,0 & 864,7 & 907,2 & 1,06 & $-0,0$ & 1964,0 & 0,0 & $-1061,634$ \\
\hline 121 & 2,0 & 865,6 & 913,0 & 1,06 & $-0,0$ & 1960,0 & 0,0 & $-3411,609$ \\
\hline 122 & 2,0 & 866,4 & 918,7 & 1,06 & $-0,0$ & 1956,0 & 0,0 & 3066,509 \\
\hline 123 & 2,0 & 867,1 & 924,2 & 1,06 & $-0,0$ & 1951,0 & 0,0 & 995,112 \\
\hline 124 & 2,0 & 867,7 & 929,6 & 1,06 & $-0,0$ & 1947,0 & 136,4 & 617,935 \\
\hline 125 & 2,0 & 868,3 & 934,9 & 1,06 & $-0,0$ & 1942,0 & 118,4 & 437,469 \\
\hline 126 & 2,0 & 868,8 & 940,0 & 1,06 & $-0,0$ & 1937,0 & 108,0 & 345,282 \\
\hline 127 & 2,0 & 869,2 & 945,0 & 1,06 & $-0,0$ & 1932,0 & 100,4 & 283,616 \\
\hline 128 & 2,0 & 869,5 & 949,8 & 1,06 & $-0,0$ & 1927,0 & 94,4 & 239,289 \\
\hline 129 & 2,0 & 869,8 & 954,5 & 1,06 & $-0,0$ & 1922,0 & 89,4 & 207,681 \\
\hline 130 & 2,0 & 870,0 & 959,1 & 1,06 & $-0,0$ & 1916,0 & 85,1 & 182,574 \\
\hline 131 & 2,0 & 870,2 & 963,5 & 1,06 & $-0,0$ & 1911,0 & 81,3 & 163,128 \\
\hline 132 & 2,0 & 870,2 & 967,7 & 1,06 & $-0,0$ & 1905,0 & 78,0 & 147,573 \\
\hline 133 & 2,0 & 870,2 & 971,8 & 1,06 & $-0,0$ & 1899,0 & 75,0 & 134,162 \\
\hline 134 & 2,0 & 870,2 & 975,8 & 1,06 & $-0,0$ & 1894,0 & 72,3 & 123,073 \\
\hline 135 & 2,0 & 870,0 & 979,5 & 1,06 & $-0,0$ & 1887,0 & 187,5 & 113,221 \\
\hline 136 & 2,0 & 869,8 & 983,2 & 1,06 & $-0,0$ & 1882,0 & 172,3 & 105,199 \\
\hline 137 & 2,0 & 869,5 & 986,6 & 1,06 & $-0,0$ & 1876,0 & 161,6 & 97,850 \\
\hline 138 & 2,0 & 869,2 & 989,9 & 1,06 & $-0,0$ & 1869,0 & 153,0 & 91,400 \\
\hline 139 & 2,0 & 868,8 & 993,0 & 1,06 & $-0,0$ & 1864,0 & 145,9 & 85,949 \\
\hline 140 & 2,0 & 868,3 & 995,9 & 1,06 & $-0,0$ & 1857,0 & 139,7 & 80,567 \\
\hline
\end{tabular}

O índice $\left(\frac{\partial Q}{\partial V}\right)$ " da barra 1 passa por zero entre os casos 27 e 28 , pois nesse caso operativo deixa de existir barras do tipo PV, ou seja, todas as barras perderam a capacidade de controle de tensão, exceto a própria barra 1. É importante lembrar que, no cálculo do índice da barra 1, assume-se temporariamente que essa barra é PQ. Assim, não há nenhuma barra com potência reativa livre para variar e as variações infinitesimais em P1 e Q1 devem ser absorvidas por variações de geração ativa e de perdas ativas e 
Tabela 5.2: Tipos das Barras - Crescimento de Carga na Barra 2

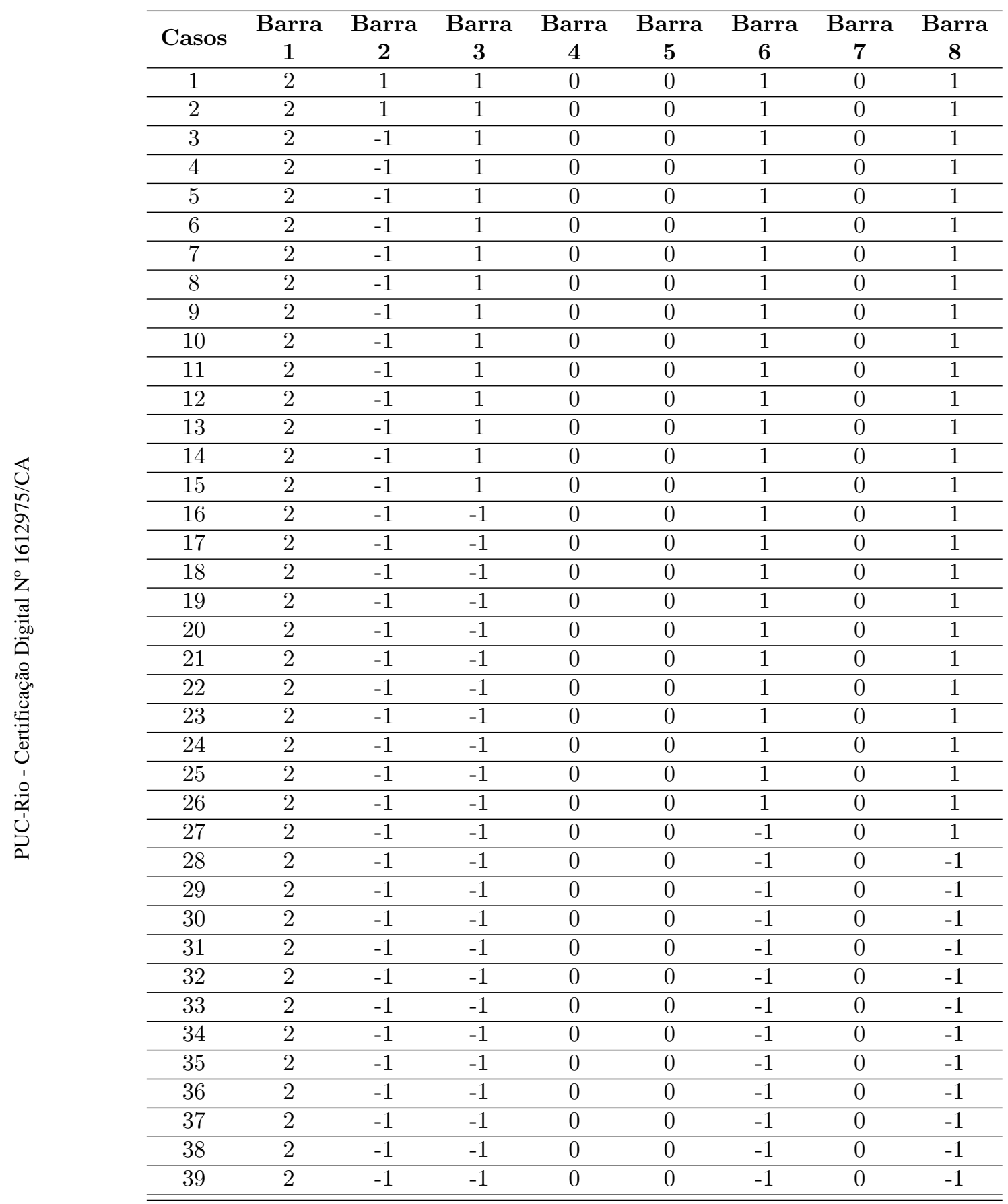


reativas. É de se esperar que o índice passe por zero e se torne negativo nos casos seguintes.

A partir do Caso 28 os índices $M_{Q V}$ e $\left(\frac{\partial Q}{\partial V}\right)$ " passam a apresentar valores negativos, muito antes da carga máxima de carregamento.

Os índices $M_{Q V}$ e $\left(\frac{\partial Q}{\partial V}\right)$ " continuam a indicar a operação do sistema no lado esquerdo da curva QV e no modo não usual de operação até o Caso 102 quando a carga máxima é atingida.

Entre os Casos 102 e 105, a carga é máxima. A margem $M_{Q V}$ vai a zero e o índice $\left(\frac{\partial Q}{\partial V}\right)$ " continua negativo pela razão já explicada. O Caso 103 apresenta anomalia no cálculo do índice $\left(\frac{\partial Q}{\partial V}\right)$ ". Provavelmente é um problema numérico já que a região é de alta não linearidade.

Na Figura 5.2 observa-se o panorama geral do comportamento do índice $\left(\frac{\partial Q}{\partial V}\right)$ " versus o aumento de carga na barra 2. Pode-se notar a deterioração do índice $\left(\frac{\partial Q}{\partial V}\right)$ " com o aumento dos patamares de carga na barra 2 e as descontinuidades que ocorrem quando da perda de controle de tensão nas barras do tipo PV.

A partir do Caso 106 a carga passa a diminuir e já não há interesse prático. Até o Caso $123 M_{Q V}$ é nula e até o Caso $121\left(\frac{\partial Q}{\partial V}\right) "$ é negativo. A partir daí não é possível explicar as mudanças de sinal e as variações de valores. Seria necessário estudar a lógica do algoritmo utilizado para o FPC, o que está fora do escopo desse trabalho.

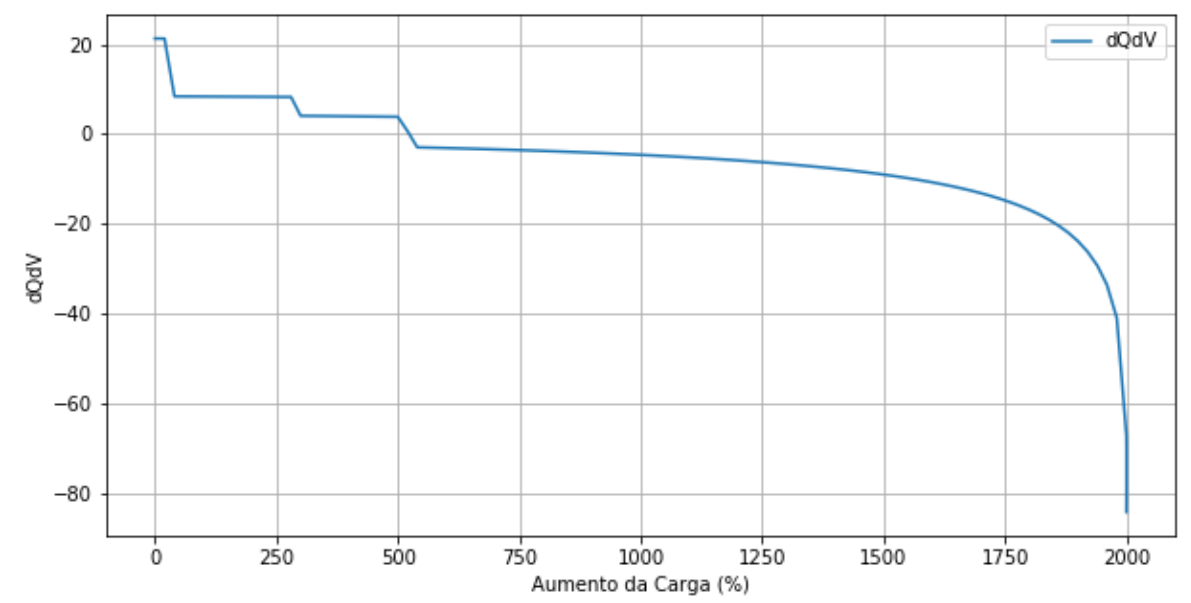

Figura 5.2: Índice $\left(\frac{\partial Q}{\partial V}\right)^{\prime \prime}$ na Barra 1 


\subsubsection{2}

\section{Análise da Barra 2}

A análise da barra 2 tem como objetivo mostrar o comportamento do índice $\left(\frac{\partial Q}{\partial V}\right)$ ", a margem e o lado da curva $Q V$ após sucessivos aumentos no patamar de carga na barra 2.

Na Tabela 5.3 apresentam-se potência reativa gerada, potência ativa e reativa de carga, tensão, ângulo, tipo da barra, e aumento percentual da carga. O índice $\left(\frac{\partial Q}{\partial V}\right)$ " e margem da curva QV $\left(M_{Q V}\right)$ também são apresentados na Tabela 5.3.

A barra 2 é do tipo PV e, portanto, a potência ativa não muda. Essa barra tem tensão controlada, até atingir seu limite de geração de potência reativa no ponto de operação 3 .

O Caso 1 correspondente ao caso-base do sistema e possui potência ativa de carga na barra 2 igual a 21,7 MW e tensão de 1,045 p.u.. Os Casos 98 ao 105 são os casos de máximo carregamento do sistema, o Caso 104 possui potência reativa de carga de 266,7 Mvar e tensão de 0,639 p.u.. A partir do Caso 105 os índices $\left(\frac{\partial Q}{\partial V}\right)$ " e margem da curva $\mathrm{QV}\left(M_{Q V}\right)$ tem valores negativos. Para a barra 2 o índice $\left(\frac{\partial Q}{\partial V}\right)$ " não captura a descontinuidade quando ocorre perda de controle de tensão na própria barra 2. Mas essa descontinuidade é percebida nos Casos 16, 27 e 28, na perda de controle de tensão em outras barras.

Tabela 5.3: Análise da Barra 2 - Crescimento de Carga na Barra 2

\begin{tabular}{cccccccccc}
\hline Casos & Tipo & $\begin{array}{c}\mathbf{Q G}_{2} \\
(\mathbf{M v a r})\end{array}$ & $\begin{array}{c}\mathbf{P L}_{2} \\
(\mathbf{M W})\end{array}$ & $\begin{array}{c}\mathbf{Q L}_{2} \\
(\mathbf{M v a r})\end{array}$ & $\begin{array}{c}\mathbf{V}_{2} \\
(\mathbf{p . u .})\end{array}$ & $\begin{array}{c}\Theta_{2} \\
\left({ }^{\circ}\right)\end{array}$ & $\begin{array}{c}\Delta \\
\text { Carga (\%) }\end{array}$ & $M_{Q V}$ & $\left(\frac{\partial Q}{\partial V}\right)^{\prime \prime}$ \\
\hline 1 & 1,0 & 43,6 & 21,7 & 12,7 & 1,045 & $-5,0$ & 0,0 & 484,5 & 30,200 \\
\hline 2 & 1,0 & 47,8 & 26,0 & 15,2 & 1,045 & $-5,1$ & 20,0 & 484,9 & 30,214 \\
\hline 3 & $-1,0$ & 50,0 & 30,4 & 17,8 & 1,044 & $-5,2$ & 40,0 & 483,3 & 30,184 \\
\hline 4 & $-1,0$ & 50,0 & 34,7 & 20,3 & 1,043 & $-5,3$ & 60,0 & 479,4 & 30,110 \\
\hline 5 & $-1,0$ & 50,0 & 39,1 & 22,9 & 1,041 & $-5,4$ & 80,0 & 475,6 & 30,033 \\
\hline 6 & $-1,0$ & 50,0 & 43,4 & 25,4 & 1,040 & $-5,6$ & 100,0 & 471,6 & 29,958 \\
\hline 7 & $-1,0$ & 50,0 & 47,7 & 27,9 & 1,039 & $-5,7$ & 120,0 & 467,7 & 29,882 \\
\hline 8 & $-1,0$ & 50,0 & 52,1 & 30,5 & 1,037 & $-5,8$ & 140,0 & 463,8 & 29,804 \\
\hline 9 & $-1,0$ & 50,0 & 56,4 & 33,0 & 1,036 & $-5,9$ & 160,0 & 459,9 & 29,727 \\
\hline 10 & $-1,0$ & 50,0 & 60,8 & 35,6 & 1,034 & $-6,0$ & 180,0 & 456,0 & 29,647 \\
\hline 11 & $-1,0$ & 50,0 & 65,1 & 38,1 & 1,033 & $-6,1$ & 200,0 & 452,0 & 29,570 \\
\hline 12 & $-1,0$ & 50,0 & 69,4 & 40,6 & 1,031 & $-6,2$ & 220,0 & 448,0 & 29,492 \\
\hline 13 & $-1,0$ & 50,0 & 73,8 & 43,2 & 1,030 & $-6,3$ & 240,0 & 443,9 & 29,410 \\
\hline 14 & $-1,0$ & 50,0 & 78,1 & 45,7 & 1,028 & $-6,4$ & 260,0 & 439,9 & 29,331 \\
\hline 15 & $-1,0$ & 50,0 & 82,5 & 48,3 & 1,027 & $-6,5$ & 280,0 & 435,9 & 29,249 \\
\hline 16 & $-1,0$ & 50,0 & 86,8 & 50,8 & 1,025 & $-6,7$ & 300,0 & 431,8 & 23,449 \\
\hline
\end{tabular}




\begin{tabular}{|c|c|c|c|c|c|c|c|c|c|}
\hline Casos & Tipo & $\begin{array}{c}\mathbf{Q G}_{2} \\
\text { (Mvar) }\end{array}$ & $\begin{array}{c}\mathbf{P L}_{2} \\
(\mathrm{MW})\end{array}$ & $\begin{array}{c}\mathrm{QL}_{2} \\
\text { (Mvar) }\end{array}$ & $\begin{array}{c}\mathrm{V}_{2} \\
\text { (p.u.) }\end{array}$ & $\begin{array}{l}\Theta_{2} \\
\left({ }^{\circ}\right) \\
\end{array}$ & $\begin{array}{c}\Delta \\
\text { Carga }(\%)\end{array}$ & $M_{Q V}$ & $\left(\frac{\partial Q}{\partial V}\right)^{\prime \prime}$ \\
\hline 17 & $-1,0$ & 50,0 & 91,1 & 53,3 & 1,023 & $-6,8$ & 320,0 & 427,7 & 23,368 \\
\hline 18 & $-1,0$ & 50,0 & 95,5 & 55,9 & 1,021 & $-6,9$ & 340,0 & 423,6 & 23,284 \\
\hline 19 & $-1,0$ & 50,0 & 99,8 & 58,4 & 1,020 & $-7,0$ & 360,0 & 419,4 & 23,201 \\
\hline 20 & $-1,0$ & 50,0 & 104,2 & 61,0 & 1,018 & $-7,1$ & 380,0 & 415,3 & 23,115 \\
\hline 21 & $-1,0$ & 50,0 & 108,5 & 63,5 & 1,016 & $-7,2$ & 400,0 & 411,1 & 23,032 \\
\hline 22 & $-1,0$ & 50,0 & 112,8 & 66,0 & 1,014 & $-7,3$ & 420,0 & 406,9 & 22,947 \\
\hline 23 & $-1,0$ & 50,0 & 117,2 & 68,6 & 1,012 & $-7,4$ & 440,0 & 402,7 & 22,859 \\
\hline 24 & $-1,0$ & 50,0 & 121,5 & 71,1 & 1,010 & $-7,5$ & 460,0 & 398,4 & 22,774 \\
\hline 25 & $-1,0$ & 50,0 & 125,9 & 73,7 & 1,008 & $-7,7$ & 480,0 & 394,2 & 22,684 \\
\hline 26 & $-1,0$ & 50,0 & 130,2 & 76,2 & 1,006 & $-7,8$ & 500,0 & 389,9 & 22,597 \\
\hline 27 & $-1,0$ & 50,0 & 134,5 & 78,7 & 1,004 & $-7,9$ & 520,0 & 385,6 & 20,641 \\
\hline 28 & $-1,0$ & 50,0 & 138,9 & 81,3 & 1,001 & $-8,0$ & 540,0 & 381,3 & 18,552 \\
\hline 29 & $-1,0$ & 50,0 & 143,2 & 83,8 & 0,999 & $-8,1$ & 560,0 & 376,9 & 18,457 \\
\hline 30 & $-1,0$ & 50,0 & 147,6 & 86,4 & 0,996 & $-8,2$ & 580,0 & 372,6 & 18,357 \\
\hline 31 & $-1,0$ & 50,0 & 151,9 & 88,9 & 0,994 & $-8,3$ & 600,0 & 368,2 & 18,259 \\
\hline 32 & $-1,0$ & 50,0 & 156,2 & 91,4 & 0,991 & $-8,4$ & 620,0 & 363,8 & 18,160 \\
\hline 33 & $-1,0$ & 50,0 & 160,6 & 94,0 & 0,989 & $-8,6$ & 640,0 & 359,3 & 18,057 \\
\hline 34 & $-1,0$ & 50,0 & 164,9 & 96,5 & 0,986 & $-8,7$ & 660,0 & 354,9 & 17,956 \\
\hline 35 & $-1,0$ & 50,0 & 169,3 & 99,1 & 0,984 & $-8,8$ & 680,0 & 350,5 & 17,851 \\
\hline 36 & $-1,0$ & 50,0 & 173,6 & 101,6 & 0,981 & $-8,9$ & 700,0 & 345,9 & 17,748 \\
\hline 37 & $-1,0$ & 50,0 & 177,9 & 104,1 & 0,979 & $-9,0$ & 720,0 & 341,4 & 17,644 \\
\hline 38 & $-1,0$ & 50,0 & 182,3 & 106,7 & 0,976 & $-9,2$ & 740,0 & 336,9 & 17,535 \\
\hline 39 & $-1,0$ & 50,0 & 186,6 & 109,2 & 0,973 & $-9,3$ & 760,0 & 332,3 & 17,429 \\
\hline 40 & $-1,0$ & 50,0 & 191,0 & 111,8 & 0,971 & $-9,4$ & 780,0 & 327,7 & 17,317 \\
\hline 41 & $-1,0$ & 50,0 & 195,3 & 114,3 & 0,968 & $-9,5$ & 800,0 & 323,1 & 17,208 \\
\hline 42 & $-1,0$ & 50,0 & 199,6 & 116,8 & 0,965 & $-9,6$ & 820,0 & 318,5 & 17,098 \\
\hline 43 & $-1,0$ & 50,0 & 204,0 & 119,4 & 0,962 & $-9,8$ & 840,0 & 313,8 & 16,983 \\
\hline 44 & $-1,0$ & 50,0 & 208,3 & 121,9 & 0,960 & $-9,9$ & 860,0 & 309,2 & 16,870 \\
\hline 45 & $-1,0$ & 50,0 & 212,7 & 124,5 & 0,957 & $-10,0$ & 880,0 & 304,5 & 16,752 \\
\hline 46 & $-1,0$ & 50,0 & 217,0 & 127,0 & 0,954 & $-10,1$ & 900,0 & 299,8 & 16,636 \\
\hline 47 & $-1,0$ & 50,0 & 221,3 & 129,5 & 0,951 & $-10,3$ & 920,0 & 295,0 & 16,518 \\
\hline 48 & $-1,0$ & 50,0 & 225,7 & 132,1 & 0,948 & $-10,4$ & 940,0 & 290,3 & 16,396 \\
\hline 49 & $-1,0$ & 50,0 & 230,0 & 134,6 & 0,945 & $-10,5$ & 960,0 & 285,5 & 16,275 \\
\hline 50 & $-1,0$ & 50,0 & 234,4 & 137,2 & 0,942 & $-10,7$ & 980,0 & 280,6 & 16,149 \\
\hline 51 & $-1,0$ & 50,0 & 238,7 & 139,7 & 0,939 & $-10,8$ & 1000,0 & 275,8 & 16,025 \\
\hline 52 & $-1,0$ & 50,0 & 243,0 & 142,2 & 0,936 & $-10,9$ & 1020,0 & 271,0 & 15,900 \\
\hline 53 & $-1,0$ & 50,0 & 247,4 & 144,8 & 0,933 & $-11,1$ & 1040,0 & 266,0 & 15,769 \\
\hline 54 & $-1,0$ & 50,0 & 251,7 & 147,3 & 0,930 & $-11,2$ & 1060,0 & 261,1 & 15,639 \\
\hline 55 & $-1,0$ & 50,0 & 256,1 & 149,9 & 0,927 & $-11,3$ & 1080,0 & 256,2 & 15,504 \\
\hline 56 & $-1,0$ & 50,0 & 260,4 & 152,4 & 0,924 & $-11,5$ & 1100,0 & 251,2 & 15,371 \\
\hline 57 & $-1,0$ & 50,0 & 264,7 & 154,9 & 0,921 & $-11,6$ & 1120,0 & 246,3 & 15,236 \\
\hline 58 & $-1,0$ & 50,0 & 269,1 & 157,5 & 0,918 & $-11,8$ & 1140,0 & 241,3 & 15,095 \\
\hline 59 & $-1,0$ & 50,0 & 273,4 & 160,0 & 0,914 & $-11,9$ & 1160,0 & 236,2 & 14,956 \\
\hline
\end{tabular}




\begin{tabular}{|c|c|c|c|c|c|c|c|c|c|}
\hline Casos & Tipo & $\begin{array}{c}\mathrm{QG}_{2} \\
\text { (Mvar) }\end{array}$ & $\begin{array}{c}\mathbf{P L}_{2} \\
(\mathrm{MW})\end{array}$ & $\begin{array}{c}\mathrm{QL}_{2} \\
\text { (Mvar) }\end{array}$ & $\begin{array}{c}\mathrm{V}_{2} \\
\text { (p.u.) }\end{array}$ & $\begin{array}{l}\Theta_{2} \\
\left({ }^{\circ}\right)\end{array}$ & $\begin{array}{c}\Delta \\
\text { Carga }(\%)\end{array}$ & $M_{Q V}$ & $\left(\frac{\partial Q}{\partial V}\right)^{\prime \prime}$ \\
\hline 60 & $-1,0$ & 50,0 & 277,8 & 162,6 & 0,911 & $-12,0$ & 1180,0 & 231,2 & 14,810 \\
\hline 61 & $-1,0$ & 50,0 & 282,1 & 165,1 & 0,908 & $-12,2$ & 1200,0 & 226,1 & $\overline{14,666}$ \\
\hline 62 & $-1,0$ & 50,0 & 286,4 & 167,6 & 0,904 & $-12,3$ & 1220,0 & 220,9 & 14,520 \\
\hline 63 & $-1,0$ & 50,0 & 290,8 & 170,2 & 0,901 & $-12,5$ & 1240,0 & 215,8 & 14,366 \\
\hline 64 & $-1,0$ & 50,0 & 295,1 & 172,7 & 0,897 & $-12,6$ & 1260,0 & 210,6 & 14,215 \\
\hline 65 & $-1,0$ & 50,0 & 299,5 & 175,3 & 0,894 & $-12,8$ & 1280,0 & 205,5 & 14,056 \\
\hline 66 & $-1,0$ & 50,0 & 303,8 & 177,8 & 0,890 & $-13,0$ & 1300,0 & 200,3 & 13,899 \\
\hline 67 & $-1,0$ & 50,0 & 308,1 & 180,3 & 0,887 & $-13,1$ & 1320,0 & 195,0 & 13,739 \\
\hline 68 & $-1,0$ & 50,0 & 312,5 & 182,9 & 0,883 & $-13,3$ & 1340,0 & 189,8 & 13,570 \\
\hline 69 & $-1,0$ & 50,0 & 316,8 & 185,4 & 0,879 & $-13,4$ & 1360,0 & 184,4 & 13,404 \\
\hline 70 & $-1,0$ & 50,0 & 321,2 & 188,0 & 0,875 & $-13,6$ & 1380,0 & 179,1 & 13,228 \\
\hline 71 & $-1,0$ & 50,0 & 325,5 & 190,5 & 0,872 & $-13,8$ & 1400,0 & 173,8 & 13,055 \\
\hline 72 & $-1,0$ & 50,0 & 329,8 & 193,0 & 0,868 & $-13,9$ & 1420,0 & 168,4 & 12,878 \\
\hline 73 & $-1,0$ & 50,0 & 334,2 & 195,6 & 0,864 & $-14,1$ & 1440,0 & 163,0 & 12,690 \\
\hline 74 & $-1,0$ & 50,0 & 338,5 & 198,1 & 0,860 & $-14,3$ & 1460,0 & 157,5 & 12,505 \\
\hline 75 & $-1,0$ & 50,0 & 342,9 & 200,7 & 0,855 & $-14,5$ & 1480,0 & 152,2 & 12,309 \\
\hline 76 & $-1,0$ & 50,0 & 347,2 & 203,2 & 0,851 & $-14,7$ & 1500,0 & 146,6 & 12,114 \\
\hline 77 & $-1,0$ & 50,0 & 351,5 & 205,7 & 0,847 & $-14,8$ & 1520,0 & 141,2 & 11,914 \\
\hline 78 & $-1,0$ & 50,0 & 355,9 & 208,3 & 0,842 & $-15,0$ & 1540,0 & 135,5 & 11,702 \\
\hline 79 & $-1,0$ & 50,0 & 360,2 & 210,8 & 0,838 & $-15,2$ & 1560,0 & 130,1 & 11,491 \\
\hline 80 & $-1,0$ & 50,0 & 364,6 & 213,4 & 0,833 & $-15,4$ & 1580,0 & 124,4 & 11,267 \\
\hline 81 & $-1,0$ & 50,0 & 368,9 & 215,9 & 0,829 & $-15,6$ & 1600,0 & 118,8 & 11,043 \\
\hline 82 & $-1,0$ & 50,0 & 373,2 & 218,4 & 0,824 & $-15,8$ & 1620,0 & 113,1 & 10,812 \\
\hline 83 & $-1,0$ & 50,0 & 377,6 & 221,0 & 0,819 & $-16,0$ & 1640,0 & 107,5 & 10,566 \\
\hline 84 & $-1,0$ & 50,0 & 381,9 & 223,5 & 0,814 & $-16,3$ & 1660,0 & 101,8 & 10,318 \\
\hline 85 & $-1,0$ & 50,0 & 386,3 & 226,1 & 0,808 & $-16,5$ & 1680,0 & 96,0 & 10,054 \\
\hline 86 & $-1,0$ & 50,0 & 390,6 & 228,6 & 0,803 & $-16,7$ & 1700,0 & 90,3 & 9,787 \\
\hline 87 & $-1,0$ & 50,0 & 394,9 & 231,1 & 0,797 & $-16,9$ & 1720,0 & 84,4 & 9,509 \\
\hline 88 & $-1,0$ & 50,0 & 399,3 & 233,7 & 0,792 & $-17,2$ & 1740,0 & 78,7 & 9,209 \\
\hline 89 & $-1,0$ & 50,0 & 403,6 & 236,2 & 0,785 & $-17,4$ & 1760,0 & 72,8 & 8,904 \\
\hline 90 & $-1,0$ & 50,0 & 408,0 & 238,8 & 0,779 & $-17,7$ & 1780,0 & 66,8 & 8,574 \\
\hline 91 & $-1,0$ & 50,0 & 412,3 & 241,3 & 0,773 & $-18,0$ & 1800,0 & 61,0 & 8,234 \\
\hline 92 & $-1,0$ & 50,0 & 416,6 & 243,8 & 0,766 & $-18,3$ & 1820,0 & 55,1 & 7,874 \\
\hline 93 & $-1,0$ & 50,0 & 421,0 & 246,4 & 0,758 & $-18,6$ & 1840,0 & 49,1 & 7,477 \\
\hline 94 & $-1,0$ & 50,0 & 425,3 & 248,9 & 0,751 & $-18,9$ & 1860,0 & 43,0 & 7,061 \\
\hline 95 & $-1,0$ & 50,0 & 429,7 & 251,5 & 0,742 & $-19,2$ & 1880,0 & 36,9 & 6,594 \\
\hline 96 & $-1,0$ & 50,0 & 434,0 & 254,0 & 0,733 & $-19,6$ & 1900,0 & 30,9 & 6,094 \\
\hline 97 & $-1,0$ & 50,0 & 438,3 & 256,5 & 0,723 & $-20,0$ & 1920,0 & 24,8 & 5,531 \\
\hline 98 & $-1,0$ & 50,0 & 442,7 & 259,1 & 0,712 & $-20,4$ & 1940,0 & 18,7 & 4,857 \\
\hline 99 & $-1,0$ & 50,0 & 447,0 & 261,6 & 0,699 & $-21,0$ & 1960,0 & 12,6 & 4,061 \\
\hline 100 & $-1,0$ & 50,0 & 451,4 & 264,2 & 0,682 & $-21,6$ & 1980,0 & 6,4 & 2,951 \\
\hline 101 & $-1,0$ & 50,0 & 455,7 & 266,7 & 0,647 & $-22,9$ & 2000,0 & 0,1 & 0,477 \\
\hline 102 & $-1,0$ & 50,0 & 455,8 & 266,7 & 0,645 & $-23,0$ & 2000,0 & 0,0 & 0,305 \\
\hline
\end{tabular}




\begin{tabular}{|c|c|c|c|c|c|c|c|c|c|}
\hline Casos & Tipo & $\begin{array}{c}\mathrm{QG}_{2} \\
\text { (Mvar) }\end{array}$ & $\begin{array}{c}\mathbf{P L}_{2} \\
(\mathrm{MW})\end{array}$ & $\begin{array}{c}\mathrm{QL}_{2} \\
\text { (Mvar) }\end{array}$ & $\begin{array}{c}\mathrm{V}_{2} \\
\text { (p.u.) }\end{array}$ & $\begin{array}{l}\Theta_{2} \\
\left(^{\circ}\right)\end{array}$ & $\begin{array}{c}\Delta \\
\text { Carga }(\%)\end{array}$ & $M_{Q V}$ & $\left(\frac{\partial Q}{\partial V}\right)^{\prime \prime}$ \\
\hline 103 & $-1,0$ & 50,0 & 455,8 & 266,8 & 0,642 & $-23,1$ & 2001,0 & 0,0 & $-29,279$ \\
\hline 104 & $-1,0$ & 50,0 & 455,8 & 266,7 & 0,639 & $-23,3$ & 2000,0 & 0,0 & $-0,310$ \\
\hline 105 & $-1,0$ & 50,0 & 455,7 & 266,7 & 0,635 & $-23,4$ & 2000,0 & $-0,1$ & $-0,494$ \\
\hline 106 & $-1,0$ & 50,0 & 455,5 & 266,6 & 0,632 & $-23,5$ & 1999,0 & $-0,4$ & $-0,851$ \\
\hline 107 & $-1,0$ & 50,0 & 455,3 & 266,5 & 0,629 & $-23,6$ & 1998,0 & $-0,6$ & $-1,105$ \\
\hline 108 & $-1,0$ & 50,0 & 455,1 & 266,3 & 0,626 & $-23,7$ & 1997,0 & $-1,0$ & $-1,386$ \\
\hline 109 & $-1,0$ & 50,0 & 454,8 & 266,1 & 0,623 & $-23,8$ & 1996,0 & $-1,5$ & $-1,677$ \\
\hline 110 & $-1,0$ & 50,0 & 454,4 & 265,9 & 0,620 & $-23,9$ & 1994,0 & $-2,0$ & $-1,978$ \\
\hline 111 & $-1,0$ & 50,0 & 454,0 & 265,7 & 0,617 & $-24,0$ & 1992,0 & $-2,6$ & $-2,247$ \\
\hline 112 & $-1,0$ & 50,0 & 453,5 & 265,4 & 0,614 & $-24,1$ & 1990,0 & $-3,3$ & $-2,577$ \\
\hline 113 & $-1,0$ & 50,0 & 452,9 & 265,1 & 0,611 & $-24,2$ & 1987,0 & $-4,1$ & $-2,915$ \\
\hline 114 & $-1,0$ & 50,0 & 452,4 & 264,7 & 0,608 & $-24,3$ & 1985,0 & $-4,9$ & $-3,237$ \\
\hline 115 & $-1,0$ & 50,0 & 451,7 & 264,4 & 0,605 & $-24,4$ & 1982,0 & $-5,8$ & $-3,567$ \\
\hline 116 & $-1,0$ & 50,0 & 451,1 & 264,0 & 0,603 & $-24,5$ & 1979,0 & $-6,8$ & $-3,889$ \\
\hline 117 & $-1,0$ & 50,0 & 450,3 & 263,6 & 0,600 & $-24,6$ & 1975,0 & $-7,8$ & $-4,257$ \\
\hline 118 & $-1,0$ & 50,0 & 449,6 & 263,1 & 0,597 & $-24,6$ & 1972,0 & $-8,9$ & $-4,620$ \\
\hline 119 & $-1,0$ & 50,0 & 448,8 & 262,6 & 0,595 & $-24,7$ & 1968,0 & $-10,0$ & $-5,002$ \\
\hline 120 & $-1,0$ & 50,0 & 447,9 & 262,1 & 0,592 & $-24,8$ & 1964,0 & $-11,3$ & $-5,405$ \\
\hline 121 & $-1,0$ & 50,0 & 447,0 & 261,6 & 0,590 & $-24,8$ & 1960,0 & $-12,6$ & $-5,806$ \\
\hline 122 & $-1,0$ & 50,0 & 446,1 & 261,1 & 0,588 & $-24,9$ & 1956,0 & $-13,8$ & $-6,205$ \\
\hline 123 & $-1,0$ & 50,0 & 445,1 & 260,5 & 0,585 & $-25,0$ & 1951,0 & $-15,2$ & $-6,665$ \\
\hline 124 & $-1,0$ & 50,0 & 444,2 & 259,9 & 0,583 & $-25,0$ & 1947,0 & $-16,6$ & $-7,103$ \\
\hline 125 & $-1,0$ & 50,0 & 443,1 & 259,3 & 0,581 & $-25,1$ & 1942,0 & $-18,1$ & $-7,604$ \\
\hline 126 & $-1,0$ & 50,0 & 442,1 & 258,7 & 0,579 & $-25,1$ & 1937,0 & $-19,6$ & $-8,087$ \\
\hline 127 & $-1,0$ & 50,0 & 441,0 & 258,1 & 0,577 & $-25,2$ & 1932,0 & $-21,1$ & $-8,613$ \\
\hline 128 & $-1,0$ & 50,0 & 439,9 & 257,4 & 0,575 & $-25,2$ & 1927,0 & $-22,7$ & $-9,194$ \\
\hline 129 & $-1,0$ & 50,0 & 438,7 & 256,8 & 0,573 & $-25,3$ & 1922,0 & $-24,3$ & $-9,793$ \\
\hline 130 & $-1,0$ & 50,0 & 437,5 & 256,1 & 0,571 & $-25,3$ & 1916,0 & $-26,0$ & $-10,461$ \\
\hline 131 & $-1,0$ & 50,0 & 436,3 & 255,4 & 0,569 & $-25,3$ & 1911,0 & $-27,7$ & $-11,167$ \\
\hline 132 & $-1,0$ & 50,0 & 435,1 & 254,7 & 0,568 & $-25,3$ & 1905,0 & $-29,4$ & $-11,919$ \\
\hline 133 & $-1,0$ & 50,0 & 433,9 & 253,9 & 0,566 & $-25,4$ & 1899,0 & $-31,1$ & $-12,773$ \\
\hline 134 & $-1,0$ & 50,0 & 432,6 & 253,2 & 0,565 & $-25,4$ & 1894,0 & $-32,9$ & $-13,691$ \\
\hline 135 & $-1,0$ & 50,0 & 431,3 & 252,4 & 0,563 & $-25,4$ & 1887,0 & $-34,6$ & $-14,751$ \\
\hline 136 & $-1,0$ & 50,0 & 430,0 & 251,7 & 0,562 & $-25,4$ & 1882,0 & $-36,4$ & $-15,860$ \\
\hline 137 & $-1,0$ & 50,0 & 428,7 & 250,9 & 0,561 & $-25,4$ & 1876,0 & $-38,3$ & $-17,166$ \\
\hline 138 & $-1,0$ & 50,0 & 427,4 & 250,1 & 0,559 & $-25,4$ & 1869,0 & $-40,2$ & $-18,642$ \\
\hline 139 & $-1,0$ & 50,0 & 426,1 & 249,4 & 0,558 & $-25,4$ & 1864,0 & $-42,1$ & $-20,238$ \\
\hline 140 & $-1,0$ & 50,0 & 424,7 & 248,6 & 0,557 & $-25,4$ & 1857,0 & $-43,9$ & $-22,281$ \\
\hline
\end{tabular}

Na Figura 5.3 observa-se o panorama geral do comportamento do índice $\left(\frac{\partial Q}{\partial V}\right)$ " versus o aumento de carga na barra 2. Pode-se notar a deterioração do índice $\left(\frac{\partial Q}{\partial V}\right)$ " com o aumento dos patamares de carga na barra 2 e as 
descontinuidades que ocorrem quando da perda de controle de tensão nas barras do tipo PV.

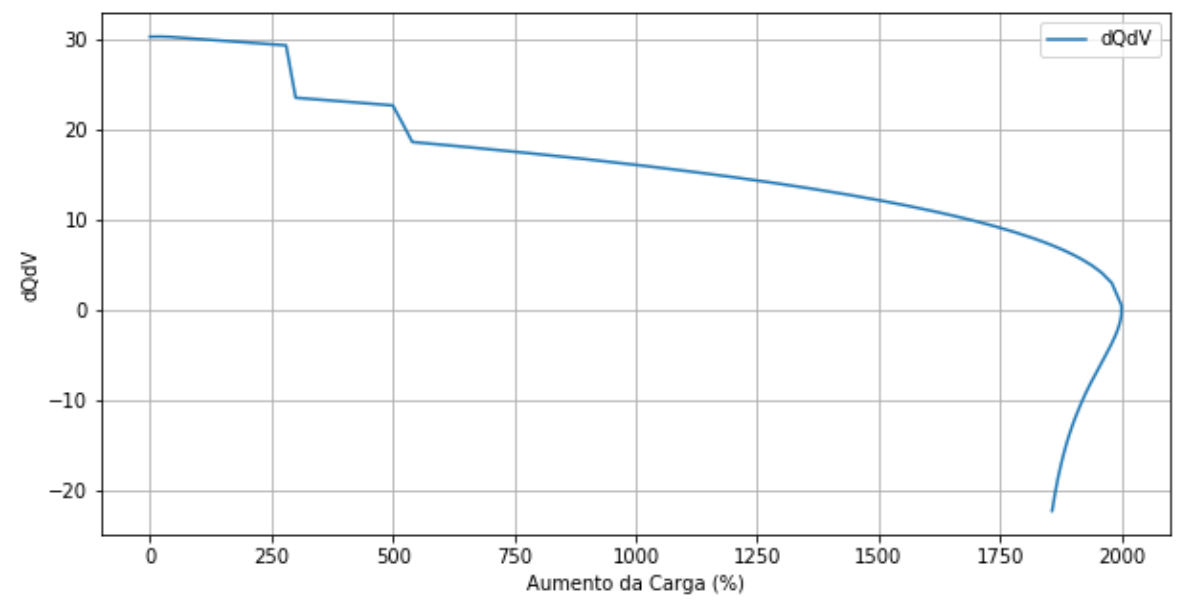

Figura 5.3: Índice $\left(\frac{\partial Q}{\partial V}\right)^{\prime}$ na Barra 2

\subsubsection{3}

\section{Análise da Barra 4}

A análise da barra 4 tem como objetivo mostrar o comportamento do índice $\left(\frac{\partial Q}{\partial V}\right)$ ", a margem e o lado da curva QV após sucessivos aumentos no patamar de carga na barra 2.

Na Tabela 5.4 apresentam-se tensão, ângulo, tipo da barra, e aumento percentual da carga. O índice $\left(\frac{\partial Q}{\partial V}\right)$ " e margem da curva $Q V\left(M_{Q V}\right)$ também são apresentados na Tabela 5.4

A barra 4 é do tipo PQ e, portanto, a carga é constante com $\mathrm{PL}=47,8$ MW e QL = -3,9 Mvar. Essa barra não fornece geração para o sistema. O Caso 1 correspondente ao caso-base do sistema e possui potência ativa de carga na barra 2 igual a 21,7 MW e tensão de 1,018 p.u. na barra 4. Os Casos 98 ao 105 são os casos de máximo carregamento do sistema, o Caso 104 possui potência reativa de carga de 266,7 Mvar na barra 2 e tensão de 0,724 p.u. na barra 4 . A partir do Caso 105 , o índice $\left(\frac{\partial Q}{\partial V}\right)$ " e margem da curva $Q V\left(M_{Q V}\right)$ indicam instabilidade do sistema com valores negativos.

Assim como para a barra 1 , na barra 4 o índice $\left(\frac{\partial Q}{\partial V}\right)$ " captura uma descontinuidade quando ocorre perda de controle de tensão nos Casos 3, 16, 27,28 . 
Tabela 5.4: Análise da Barra 4 - Crescimento de Carga na Barra 2

\begin{tabular}{|c|c|c|c|c|c|c|}
\hline Casos & Tipo & $\begin{array}{c}\mathrm{V}_{4} \\
\text { (p.u.) }\end{array}$ & $\begin{array}{l}\Theta_{4} \\
\left({ }^{\circ}\right)\end{array}$ & $\begin{array}{c}\Delta \\
\text { Carga (\%) }\end{array}$ & $M_{Q V}$ & $\left(\frac{\partial Q}{\partial V}\right)^{\prime \prime}$ \\
\hline 1 & 0,0 & 1,018 & $-10,3$ & 0,0 & 238,0 & 24,836 \\
\hline 2 & 0,0 & 1,018 & $-10,4$ & 20,0 & 236,7 & 24,835 \\
\hline 3 & 0,0 & 1,017 & $-10,5$ & 40,0 & 235,4 & 22,091 \\
\hline 4 & 0,0 & 1,017 & $-10,6$ & 60,0 & 234,1 & 22,075 \\
\hline 5 & 0,0 & 1,016 & $-10,7$ & 80,0 & 232,8 & 22,059 \\
\hline 6 & 0,0 & 1,016 & $-10,8$ & 100,0 & 231,5 & 22,043 \\
\hline 7 & 0,0 & 1,015 & $-10,9$ & 120,0 & 230,2 & 22,027 \\
\hline 8 & 0,0 & 1,015 & $-11,0$ & 140,0 & 228,8 & 22,010 \\
\hline 9 & 0,0 & 1,014 & $-11,1$ & 160,0 & 227,5 & 21,994 \\
\hline 10 & 0,0 & 1,013 & $-11,3$ & 180,0 & 226,1 & 21,976 \\
\hline 11 & 0,0 & 1,013 & $-11,4$ & 200,0 & 224,8 & 21,960 \\
\hline 12 & 0,0 & 1,012 & $-11,5$ & 220,0 & 223,4 & 21,943 \\
\hline 13 & 0,0 & 1,012 & $-11,6$ & 240,0 & 222,0 & 21,925 \\
\hline 14 & 0,0 & 1,011 & $-11,7$ & 260,0 & 220,6 & 21,908 \\
\hline 15 & 0,0 & 1,010 & $-11,8$ & 280,0 & 219,2 & 21,891 \\
\hline 16 & 0,0 & 1,010 & $-11,9$ & 300,0 & 217,8 & 16,520 \\
\hline 17 & 0,0 & 1,009 & $-12,0$ & 320,0 & 216,4 & 16,495 \\
\hline 18 & 0,0 & 1,007 & $-12,1$ & 340,0 & 214,9 & 16,468 \\
\hline 19 & 0,0 & 1,006 & $-12,2$ & 360,0 & 213,5 & 16,442 \\
\hline 20 & 0,0 & 1,005 & $-12,3$ & 380,0 & 212,0 & 16,415 \\
\hline 21 & 0,0 & 1,004 & $-12,5$ & 400,0 & 210,6 & 16,388 \\
\hline 22 & 0,0 & 1,003 & $-12,6$ & 420,0 & 209,1 & 16,361 \\
\hline 23 & 0,0 & 1,002 & $-12,7$ & 440,0 & 207,6 & 16,334 \\
\hline 24 & 0,0 & 1,001 & $-12,8$ & 460,0 & 206,1 & 16,306 \\
\hline 25 & 0,0 & 0,999 & $-12,9$ & 480,0 & 204,6 & 16,278 \\
\hline 26 & 0,0 & 0,998 & $-13,0$ & 500,0 & 203,1 & 16,250 \\
\hline 27 & 0,0 & 0,997 & $-13,1$ & 520,0 & 201,5 & 12,782 \\
\hline 28 & 0,0 & 0,995 & $-13,3$ & 540,0 & 199,9 & 9,531 \\
\hline 29 & 0,0 & 0,993 & $-13,4$ & 560,0 & 198,4 & 9,496 \\
\hline 30 & 0,0 & 0,991 & $-13,5$ & 580,0 & 196,8 & 9,460 \\
\hline 31 & 0,0 & 0,988 & $-13,6$ & 600,0 & 195,2 & 9,425 \\
\hline 32 & 0,0 & 0,986 & $-13,7$ & 620,0 & 193,6 & 9,389 \\
\hline 33 & 0,0 & 0,984 & $-13,8$ & 640,0 & 192,0 & 9,351 \\
\hline 34 & 0,0 & 0,982 & $-14,0$ & 660,0 & 190,3 & 9,314 \\
\hline 35 & 0,0 & 0,979 & $-14,1$ & 680,0 & 188,7 & 9,275 \\
\hline 36 & 0,0 & 0,977 & $-14,2$ & 700,0 & 187,0 & 9,237 \\
\hline 37 & 0,0 & 0,975 & $-14,3$ & 720,0 & 185,3 & 9,199 \\
\hline 38 & 0,0 & 0,973 & $-14,4$ & 740,0 & 183,6 & 9,159 \\
\hline 39 & 0,0 & 0,970 & $-14,6$ & 760,0 & 181,9 & 9,119 \\
\hline 40 & 0,0 & 0,968 & $-14,7$ & 780,0 & 180,1 & 9,078 \\
\hline 41 & 0,0 & 0,965 & $-14,8$ & 800,0 & 178,4 & 9,037 \\
\hline
\end{tabular}




\begin{tabular}{|c|c|c|c|c|c|c|}
\hline Casos & Tipo & $\begin{array}{c}\mathrm{V}_{4} \\
\text { (p.u.) }\end{array}$ & $\begin{array}{l}\Theta_{4} \\
\left(^{\circ}\right)\end{array}$ & $\begin{array}{c}\Delta \\
\text { Carga (\%) }\end{array}$ & $M_{Q V}$ & $\left(\frac{\partial Q}{\partial V}\right)^{\prime}$ \\
\hline 42 & 0,0 & 0,963 & $-14,9$ & 820,0 & 176,6 & 8,996 \\
\hline 43 & 0,0 & 0,961 & $-15,1$ & 840,0 & 174,8 & 8,953 \\
\hline 44 & 0,0 & 0,958 & $-15,2$ & 860,0 & 173,0 & 8,911 \\
\hline 45 & 0,0 & 0,956 & $-15,3$ & 880,0 & 171,2 & 8,866 \\
\hline 46 & 0,0 & 0,953 & $-15,5$ & 900,0 & 169,3 & 8,823 \\
\hline 47 & 0,0 & 0,951 & $-15,6$ & 920,0 & 167,4 & 8,779 \\
\hline 48 & 0,0 & 0,948 & $-15,7$ & 940,0 & 165,5 & 8,732 \\
\hline 49 & 0,0 & 0,945 & $-15,9$ & 960,0 & 163,6 & 8,686 \\
\hline 50 & 0,0 & 0,943 & $-16,0$ & 980,0 & 161,7 & 8,638 \\
\hline 51 & 0,0 & 0,940 & $-16,1$ & 1000,0 & 159,7 & 8,591 \\
\hline 52 & 0,0 & 0,937 & $-16,3$ & 1020,0 & 157,7 & 8,543 \\
\hline 53 & 0,0 & 0,935 & $-16,4$ & 1040,0 & 155,7 & 8,493 \\
\hline 54 & 0,0 & 0,932 & $-16,6$ & 1060,0 & 153,7 & 8,443 \\
\hline 55 & 0,0 & 0,929 & $-16,7$ & 1080,0 & 151,6 & 8,390 \\
\hline 56 & 0,0 & 0,926 & $-16,8$ & 1100,0 & 149,5 & 8,339 \\
\hline 57 & 0,0 & 0,923 & $-17,0$ & 1120,0 & 147,5 & 8,286 \\
\hline 58 & 0,0 & 0,921 & $-17,1$ & 1140,0 & 145,3 & 8,231 \\
\hline 59 & 0,0 & 0,918 & $-17,3$ & 1160,0 & 143,1 & 8,176 \\
\hline 60 & 0,0 & 0,915 & $-17,4$ & 1180,0 & 140,9 & 8,119 \\
\hline 61 & 0,0 & 0,912 & $-17,6$ & 1200,0 & 138,7 & 8,062 \\
\hline 62 & 0,0 & 0,908 & $-17,8$ & 1220,0 & 136,4 & 8,004 \\
\hline 63 & 0,0 & 0,905 & $-17,9$ & 1240,0 & 134,1 & 7,943 \\
\hline 64 & 0,0 & 0,902 & $-18,1$ & 1260,0 & 131,8 & 7,882 \\
\hline 65 & 0,0 & 0,899 & $-18,3$ & 1280,0 & 129,4 & 7,818 \\
\hline 66 & 0,0 & 0,896 & $-18,4$ & 1300,0 & 127,0 & 7,754 \\
\hline 67 & 0,0 & 0,892 & $-18,6$ & 1320,0 & 124,6 & 7,689 \\
\hline 68 & 0,0 & 0,889 & $-18,8$ & 1340,0 & 122,0 & 7,621 \\
\hline 69 & 0,0 & 0,886 & $-18,9$ & 1360,0 & 119,5 & 7,552 \\
\hline 70 & 0,0 & 0,882 & $-19,1$ & 1380,0 & 117,0 & 7,480 \\
\hline 71 & 0,0 & 0,879 & $-19,3$ & 1400,0 & 114,3 & 7,408 \\
\hline 72 & 0,0 & 0,875 & $-19,5$ & 1420,0 & 111,7 & 7,334 \\
\hline 73 & 0,0 & 0,871 & $-19,7$ & 1440,0 & 108,9 & 7,256 \\
\hline 74 & 0,0 & 0,867 & $-19,9$ & 1460,0 & 106,2 & 7,177 \\
\hline 75 & 0,0 & 0,864 & $-20,1$ & 1480,0 & 103,3 & 7,094 \\
\hline 76 & 0,0 & 0,860 & $-20,3$ & 1500,0 & 100,5 & 7,010 \\
\hline 77 & 0,0 & 0,856 & $-20,5$ & 1520,0 & 97,5 & 6,924 \\
\hline 78 & 0,0 & 0,852 & $-20,7$ & 1540,0 & 94,6 & 6,832 \\
\hline 79 & 0,0 & 0,847 & $-20,9$ & 1560,0 & 91,5 & 6,740 \\
\hline 80 & 0,0 & 0,843 & $-21,1$ & 1580,0 & 88,4 & 6,641 \\
\hline 81 & 0,0 & 0,839 & $-21,4$ & 1600,0 & 85,2 & 6,541 \\
\hline 82 & 0,0 & 0,834 & $-21,6$ & 1620,0 & 81,9 & 6,437 \\
\hline 83 & 0,0 & 0,829 & $-21,8$ & 1640,0 & 78,6 & 6,325 \\
\hline 84 & 0,0 & 0,824 & $-22,1$ & 1660,0 & 75,1 & 6,212 \\
\hline
\end{tabular}




\begin{tabular}{|c|c|c|c|c|c|c|}
\hline Casos & Tipo & $\begin{array}{c}\mathrm{V}_{4} \\
\text { (p.u.) }\end{array}$ & $\begin{array}{l}\Theta_{4} \\
\left(^{\circ}\right)\end{array}$ & $\begin{array}{c}\Delta \\
\text { Carga (\%) }\end{array}$ & $M_{Q V}$ & $\left(\frac{\partial Q}{\partial V}\right)^{" \prime}$ \\
\hline 85 & 0,0 & 0,819 & $-22,3$ & 1680,0 & 71,6 & 6,089 \\
\hline 86 & 0,0 & 0,814 & $-22,6$ & 1700,0 & 68,0 & 5,964 \\
\hline 87 & 0,0 & 0,809 & $-22,9$ & 1720,0 & 64,3 & 5,832 \\
\hline 88 & 0,0 & 0,803 & $-23,2$ & 1740,0 & 60,6 & 5,689 \\
\hline 89 & 0,0 & 0,797 & $-23,5$ & 1760,0 & 56,7 & 5,540 \\
\hline 90 & 0,0 & 0,791 & $-23,8$ & 1780,0 & 52,7 & 5,377 \\
\hline 91 & 0,0 & 0,785 & $-24,1$ & 1800,0 & 48,6 & 5,207 \\
\hline 92 & 0,0 & 0,778 & $-24,4$ & 1820,0 & 44,4 & 5,023 \\
\hline 93 & 0,0 & 0,771 & $-24,8$ & 1840,0 & 40,0 & 4,816 \\
\hline 94 & 0,0 & 0,763 & $-25,2$ & 1860,0 & 35,6 & 4,596 \\
\hline 95 & 0,0 & 0,755 & $-25,6$ & 1880,0 & 31,0 & 4,343 \\
\hline 96 & 0,0 & 0,746 & $-26,1$ & 1900,0 & 26,2 & 4,064 \\
\hline 97 & 0,0 & 0,736 & $-26,6$ & 1920,0 & 21,3 & 3,741 \\
\hline 98 & 0,0 & 0,724 & $-27,2$ & 1940,0 & 16,3 & 3,342 \\
\hline 99 & 0,0 & 0,710 & $-27,9$ & 1960,0 & 11,1 & 2,851 \\
\hline 100 & 0,0 & 0,692 & $-28,9$ & 1980,0 & 5,7 & 2,129 \\
\hline 101 & 0,0 & 0,653 & $-30,9$ & 2000,0 & 0,1 & 0,364 \\
\hline 102 & 0,0 & 0,651 & $-31,0$ & 2000,0 & 0,0 & 0,233 \\
\hline 103 & 0,0 & 0,647 & $-31,2$ & 2001,0 & 0,0 & $-157,501$ \\
\hline 104 & 0,0 & 0,643 & $-31,4$ & 2000,0 & 0,0 & $-0,240$ \\
\hline 105 & 0,0 & 0,639 & $-31,6$ & 2000,0 & $-0,1$ & $-0,383$ \\
\hline 106 & 0,0 & 0,635 & $-31,8$ & 1999,0 & $-0,3$ & $-0,663$ \\
\hline 107 & 0,0 & 0,632 & $-32,0$ & 1998,0 & $-0,5$ & $-0,864$ \\
\hline 108 & 0,0 & 0,628 & $-32,2$ & 1997,0 & $-0,9$ & $-1,088$ \\
\hline 109 & 0,0 & 0,624 & $-32,4$ & 1996,0 & $-1,3$ & $-1,322$ \\
\hline 110 & 0,0 & 0,620 & $-32,6$ & 1994,0 & $-1,8$ & $-1,564$ \\
\hline 111 & 0,0 & 0,616 & $-32,8$ & 1992,0 & $-2,4$ & $-1,781$ \\
\hline 112 & 0,0 & 0,613 & $-33,0$ & 1990,0 & $-3,0$ & $-2,047$ \\
\hline 113 & 0,0 & 0,609 & $-33,2$ & 1987,0 & $-3,6$ & $-2,321$ \\
\hline 114 & 0,0 & 0,605 & $-33,4$ & 1985,0 & $-4,4$ & $-2,581$ \\
\hline 115 & 0,0 & 0,602 & $-33,6$ & 1982,0 & $-5,2$ & $-2,848$ \\
\hline 116 & 0,0 & 0,598 & $-33,8$ & 1979,0 & $-6,0$ & $-3,106$ \\
\hline 117 & 0,0 & 0,594 & $\begin{array}{l}-33,9 \\
\end{array}$ & 1975,0 & $-6,9$ & $-3,399$ \\
\hline 118 & 0,0 & 0,591 & $-34,1$ & 1972,0 & $-7,9$ & $-3,685$ \\
\hline 119 & 0,0 & 0,587 & $-34,3$ & 1968,0 & $-8,9$ & $-3,983$ \\
\hline 120 & 0,0 & 0,584 & $-34,5$ & 1964,0 & $-9,9$ & $-4,294$ \\
\hline 121 & 0,0 & 0,580 & $-34,7$ & 1960,0 & $-11,0$ & $-4,596$ \\
\hline 122 & 0,0 & 0,577 & $-34,8$ & 1956,0 & $-12,2$ & $-4,893$ \\
\hline 123 & 0,0 & 0,573 & $-35,0$ & 1951,0 & $-13,3$ & $-5,226$ \\
\hline 124 & 0,0 & 0,570 & $-35,2$ & 1947,0 & $-14,5$ & $-5,535$ \\
\hline 125 & 0,0 & 0,566 & $-35,3$ & 1942,0 & $-15,7$ & $-5,877$ \\
\hline 126 & 0,0 & 0,563 & $-35,5$ & 1937,0 & $-17,0$ & $-6,196$ \\
\hline 127 & 0,0 & 0,560 & $-35,6$ & 1932,0 & $-18,3$ & $-6,531$ \\
\hline
\end{tabular}




\begin{tabular}{ccccccc}
\hline Casos & Tipo & $\begin{array}{c}\mathbf{V}_{4} \\
(\mathbf{p . u} .)\end{array}$ & $\begin{array}{c}\Theta_{4} \\
\left({ }^{\circ}\right)\end{array}$ & $\begin{array}{c}\Delta \\
\text { Carga (\%) }\end{array}$ & $M_{Q V}$ & $\left(\frac{\partial Q}{\partial V}\right)^{\prime \prime}$ \\
\hline 128 & 0,0 & 0,556 & $-35,8$ & 1927,0 & $-19,5$ & $-6,885$ \\
\hline 129 & 0,0 & 0,553 & $-35,9$ & 1922,0 & $-20,9$ & $-7,232$ \\
\hline 130 & 0,0 & 0,550 & $-36,1$ & 1916,0 & $-22,2$ & $-7,599$ \\
\hline 131 & 0,0 & 0,547 & $-36,2$ & 1911,0 & $-23,6$ & $-7,965$ \\
\hline 132 & 0,0 & 0,544 & $-36,4$ & 1905,0 & $-24,9$ & $-8,329$ \\
\hline 133 & 0,0 & 0,541 & $-36,5$ & 1899,0 & $-26,3$ & $-8,715$ \\
\hline 134 & 0,0 & 0,538 & $-36,6$ & 1894,0 & $-27,7$ & $-9,096$ \\
\hline 135 & 0,0 & 0,535 & $-36,7$ & 1887,0 & $-29,2$ & $-9,499$ \\
\hline 136 & 0,0 & 0,532 & $-36,9$ & 1882,0 & $-30,6$ & $-9,881$ \\
\hline 137 & 0,0 & 0,529 & $-37,0$ & 1876,0 & $-31,9$ & $-10,286$ \\
\hline 138 & 0,0 & 0,526 & $-37,1$ & 1869,0 & $-33,4$ & $-10,693$ \\
\hline 139 & 0,0 & 0,523 & $-37,2$ & 1864,0 & $-34,8$ & $-11,080$ \\
\hline 140 & 0,0 & 0,521 & $-37,3$ & 1857,0 & $-36,2$ & $-11,510$ \\
\hline
\end{tabular}

Na Figura 5.4 observa-se o panorama geral do comportamento do índice $\left(\frac{\partial Q}{\partial V}\right)$ " versus o aumento de carga na barra 4. Pode-se notar a deterioração do índice $\left(\frac{\partial Q}{\partial V}\right)$ " com o aumento dos patamares de carga na barra 2 e as descontinuidades que ocorrem quando da perda de controle de tensão nas barras do tipo PV.

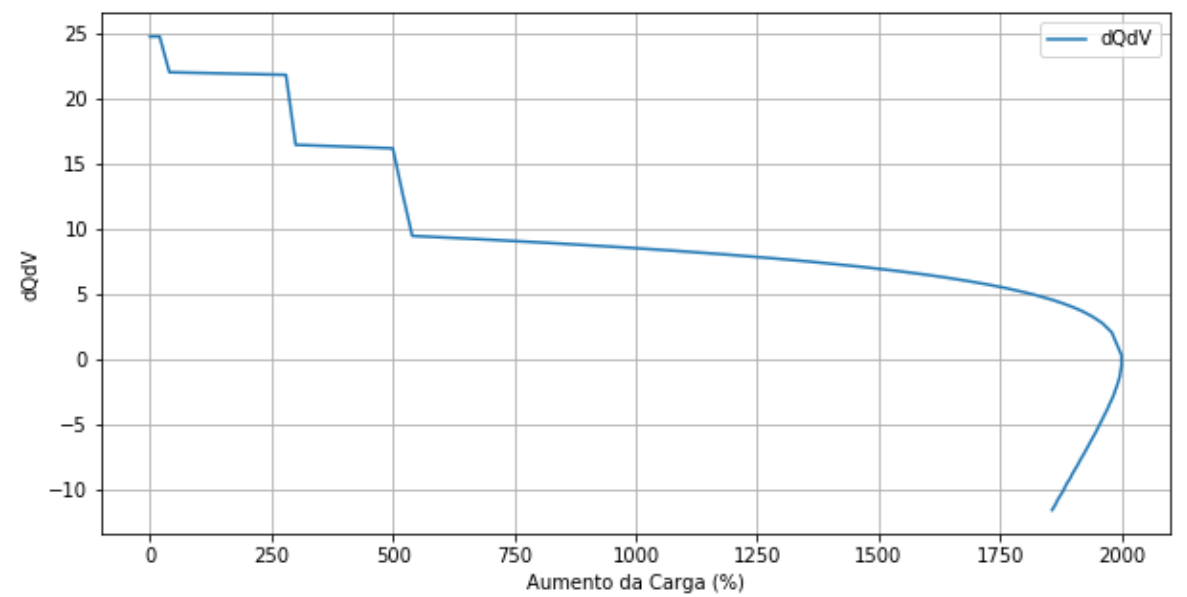

Figura 5.4: Índice $\left(\frac{\partial Q}{\partial V}\right) "$ na Barra 4

\subsubsection{4}

\section{Análise da Barra 5}

A análise da barra 5 tem como objetivo mostrar o comportamento do índice $\left(\frac{\partial Q}{\partial V}\right)$ ", a margem e o lado da curva $Q V$ após sucessivos aumentos no 
patamar de carga na barra 2 .

Na Tabela 5.5 apresentam-se tensão, ângulo, tipo da barra, e aumento percentual da carga. O índice $\left(\frac{\partial Q}{\partial V}\right)$ " e margem da curva $\mathrm{QV}\left(M_{Q V}\right)$ também são apresentados na Tabela 5.5.

A barra 5 é do tipo PQ e, portanto, a carga é constante com $\mathrm{PL}=7,6 \mathrm{MW}$ e $\mathrm{QL}=1,6$ Mvar. Essa barra não fornece geração. O Caso 1 correspondente ao caso-base do sistema e possui potência ativa de carga na barra 2 igual a 21,7 MW e tensão de 1,02 p.u. na barra 5. Os Casos 98 ao 105 são os casos de máximo carregamento do sistema, o Caso 104 possui potência reativa de carga de 266,7 Mvar na barra 2 e tensão de 0,672 p.u. na barra 5. A partir do Caso 105 o índice $\left(\frac{\partial Q}{\partial V}\right)$ " e margem da curva QV $\left(M_{Q V}\right)$ tem valores negativos. Assim como para a barra 1 , na barra 5 o índice $\left(\frac{\partial Q}{\partial V}\right)$ " captura uma descontinuidade quando ocorre perda de controle de tensão nos Casos 3, 16, 27, 28.

Tabela 5.5: Análise da Barra 5 - Crescimento de Carga na Barra 2

\begin{tabular}{ccccccc}
\hline Casos & Tipo & $\begin{array}{c}\mathbf{V}_{5} \\
\text { (p.u.) }\end{array}$ & $\begin{array}{c}\Theta_{5} \\
\left({ }^{\circ}\right)\end{array}$ & $\begin{array}{c}\Delta \\
\text { Carga }(\mathbf{\%})\end{array}$ & $M_{Q V}$ & $\left(\frac{\partial Q}{\partial V}\right)^{\prime}$ \\
\hline 1 & 0,0 & 1,020 & $-8,8$ & 0,0 & 259,4 & 24,294 \\
\hline 2 & 0,0 & 1,019 & $-8,9$ & 20,0 & 258,0 & 24,293 \\
\hline 3 & 0,0 & 1,019 & $-9,0$ & 40,0 & 256,6 & 21,563 \\
\hline 4 & 0,0 & 1,019 & $-9,1$ & 60,0 & 255,2 & 21,546 \\
\hline 5 & 0,0 & 1,018 & $-9,1$ & 80,0 & 253,8 & 21,529 \\
\hline 6 & 0,0 & 1,017 & $-9,2$ & 100,0 & 252,4 & 21,511 \\
\hline 7 & 0,0 & 1,017 & $-9,3$ & 120,0 & 251,0 & 21,494 \\
\hline 8 & 0,0 & 1,016 & $-9,4$ & 140,0 & 249,6 & 21,476 \\
\hline 9 & 0,0 & 1,016 & $-9,5$ & 160,0 & 248,1 & 21,458 \\
\hline 10 & 0,0 & 1,015 & $-9,6$ & 180,0 & 246,7 & 21,440 \\
\hline 11 & 0,0 & 1,014 & $-9,7$ & 200,0 & 245,3 & 21,422 \\
\hline 12 & 0,0 & 1,014 & $-9,8$ & 220,0 & 243,8 & 21,404 \\
\hline 13 & 0,0 & 1,013 & $-9,9$ & 240,0 & 242,3 & 21,385 \\
\hline 14 & 0,0 & 1,013 & $-10,0$ & 260,0 & 240,8 & 21,367 \\
\hline 15 & 0,0 & 1,012 & $-10,1$ & 280,0 & 239,3 & 21,348 \\
\hline 16 & 0,0 & 1,011 & $-10,2$ & 300,0 & 237,8 & 18,193 \\
\hline 17 & 0,0 & 1,010 & $-10,3$ & 320,0 & 236,3 & 18,166 \\
\hline 18 & 0,0 & 1,009 & $-10,4$ & 340,0 & 234,8 & 18,139 \\
\hline 19 & 0,0 & 1,008 & $-10,5$ & 360,0 & 233,2 & 18,112 \\
\hline 20 & 0,0 & 1,007 & $-10,6$ & 380,0 & 231,6 & 18,083 \\
\hline 21 & 0,0 & 1,006 & $-10,7$ & 400,0 & 230,1 & 18,056 \\
\hline 22 & 0,0 & 1,005 & $-10,8$ & 420,0 & 228,5 & 18,028 \\
\hline 23 & 0,0 & 1,004 & $-10,9$ & 440,0 & 226,8 & 17,999 \\
\hline 24 & 0,0 & 1,003 & $-11,0$ & 460,0 & 225,2 & 17,970 \\
\hline 25 & 0,0 & 1,002 & $-11,1$ & 480,0 & 223,6 & 17,941 \\
\hline & & & & & &
\end{tabular}




\begin{tabular}{|c|c|c|c|c|c|c|}
\hline Casos & Tipo & $\begin{array}{c}\mathbf{V}_{5} \\
\text { (p.u.) }\end{array}$ & $\begin{array}{l}\Theta_{5} \\
\left({ }^{\circ}\right)\end{array}$ & $\begin{array}{c}\Delta \\
\text { Carga (\%) }\end{array}$ & $M_{Q V}$ & $\left(\frac{\partial Q}{\partial V}\right)^{\prime \prime}$ \\
\hline 26 & 0,0 & 1,001 & $-11,2$ & 500,0 & 221,9 & 17,912 \\
\hline 27 & 0,0 & 0,999 & $-11,3$ & 520,0 & 220,3 & 13,179 \\
\hline 28 & 0,0 & 0,998 & $-11,4$ & 540,0 & 218,6 & 10,505 \\
\hline 29 & 0,0 & 0,996 & $-11,5$ & 560,0 & 216,9 & 10,469 \\
\hline 30 & 0,0 & 0,994 & $-11,6$ & 580,0 & 215,2 & 10,430 \\
\hline 31 & 0,0 & 0,992 & $-11,7$ & 600,0 & 213,5 & 10,393 \\
\hline 32 & 0,0 & 0,990 & $-11,8$ & 620,0 & 211,7 & 10,355 \\
\hline 33 & 0,0 & 0,988 & $-11,9$ & 640,0 & 210,0 & 10,315 \\
\hline 34 & 0,0 & 0,986 & $-12,0$ & 660,0 & 208,2 & 10,276 \\
\hline 35 & 0,0 & 0,984 & $-12,1$ & 680,0 & 206,4 & 10,235 \\
\hline 36 & 0,0 & 0,981 & $-12,2$ & 700,0 & 204,6 & 10,195 \\
\hline 37 & 0,0 & 0,979 & $-12,3$ & 720,0 & 202,8 & 10,155 \\
\hline 38 & 0,0 & 0,977 & $-12,4$ & 740,0 & 200,9 & 10,112 \\
\hline 39 & 0,0 & 0,975 & $-12,5$ & 760,0 & 199,1 & 10,071 \\
\hline 40 & 0,0 & 0,973 & $-12,7$ & 780,0 & 197,1 & 10,027 \\
\hline 41 & 0,0 & 0,971 & $-12,8$ & 800,0 & 195,2 & 9,984 \\
\hline 42 & 0,0 & 0,968 & $-12,9$ & 820,0 & 193,3 & 9,940 \\
\hline 43 & 0,0 & 0,966 & $-13,0$ & 840,0 & 191,4 & 9,895 \\
\hline 44 & 0,0 & 0,964 & $-13,1$ & 860,0 & 189,4 & 9,850 \\
\hline 45 & 0,0 & 0,962 & $-13,2$ & 880,0 & 187,4 & 9,803 \\
\hline 46 & 0,0 & 0,959 & $-13,3$ & 900,0 & 185,4 & 9,757 \\
\hline 47 & 0,0 & 0,957 & $-13,4$ & 920,0 & 183,4 & 9,710 \\
\hline 48 & 0,0 & 0,955 & $-13,5$ & 940,0 & 181,3 & 9,660 \\
\hline 49 & 0,0 & 0,952 & $-13,7$ & 960,0 & 179,2 & 9,612 \\
\hline 50 & 0,0 & 0,950 & $-13,8$ & 980,0 & 177,1 & 9,561 \\
\hline 51 & 0,0 & 0,947 & $-13,9$ & 1000,0 & 174,9 & 9,510 \\
\hline 52 & 0,0 & 0,945 & $-14,0$ & 1020,0 & 172,7 & 9,459 \\
\hline 53 & 0,0 & 0,942 & $-14,1$ & 1040,0 & 170,5 & 9,406 \\
\hline 54 & 0,0 & 0,940 & $-14,3$ & 1060,0 & 168,3 & 9,353 \\
\hline 55 & 0,0 & 0,937 & $-14,4$ & 1080,0 & 166,1 & 9,297 \\
\hline 56 & 0,0 & 0,935 & $-14,5$ & 1100,0 & 163,8 & 9,242 \\
\hline 57 & 0,0 & 0,932 & $-14,6$ & 1120,0 & 161,5 & 9,186 \\
\hline 58 & 0,0 & 0,929 & $-14,8$ & 1140,0 & 159,1 & 9,127 \\
\hline 59 & 0,0 & 0,926 & $-14,9$ & 1160,0 & 156,7 & 9,069 \\
\hline 60 & 0,0 & 0,924 & $-15,0$ & 1180,0 & 154,3 & 9,007 \\
\hline 61 & 0,0 & 0,921 & $-15,2$ & 1200,0 & 151,9 & 8,946 \\
\hline 62 & 0,0 & 0,918 & $-15,3$ & 1220,0 & 149,3 & 8,884 \\
\hline 63 & 0,0 & 0,915 & $-15,4$ & 1240,0 & 146,8 & 8,819 \\
\hline 64 & 0,0 & 0,912 & $-15,6$ & 1260,0 & 144,3 & 8,754 \\
\hline 65 & 0,0 & 0,909 & $-15,7$ & 1280,0 & 141,6 & 8,685 \\
\hline 66 & 0,0 & 0,906 & $-15,8$ & 1300,0 & 139,0 & 8,617 \\
\hline 67 & 0,0 & 0,903 & $-16,0$ & 1320,0 & 136,3 & 8,548 \\
\hline 68 & 0,0 & 0,900 & $-16,1$ & 1340,0 & 133,5 & 8,474 \\
\hline
\end{tabular}




\begin{tabular}{|c|c|c|c|c|c|c|}
\hline Casos & Tipo & $\begin{array}{c}\mathrm{V}_{5} \\
\text { (p.u.) }\end{array}$ & $\begin{array}{l}\Theta_{5} \\
\left({ }^{\circ}\right) \\
\end{array}$ & $\begin{array}{c}\Delta \\
\text { Carga (\%) } \\
\end{array}$ & $M_{Q V}$ & $\left(\frac{\partial Q}{\partial V}\right)$ \\
\hline 69 & 0,0 & 0,897 & $-16,3$ & 1360,0 & 130,8 & 8,400 \\
\hline 70 & 0,0 & 0,894 & $-16,4$ & 1380,0 & 127,9 & 8,323 \\
\hline 71 & 0,0 & 0,890 & $-16,6$ & 1400,0 & 125,0 & 8,245 \\
\hline 72 & 0,0 & 0,887 & $-16,7$ & 1420,0 & 122,1 & 8,166 \\
\hline 73 & 0,0 & 0,884 & $-16,9$ & 1440,0 & 119,0 & 8,081 \\
\hline 74 & 0,0 & 0,880 & $-17,1$ & 1460,0 & 116,0 & 7,997 \\
\hline 75 & 0,0 & 0,877 & $-17,2$ & 1480,0 & 112,9 & 7,907 \\
\hline 76 & 0,0 & 0,873 & $-17,4$ & 1500,0 & 109,7 & 7,817 \\
\hline 77 & 0,0 & 0,869 & $-17,6$ & 1520,0 & 106,5 & 7,723 \\
\hline 78 & 0,0 & 0,865 & $-17,7$ & 1540,0 & 103,2 & 7,624 \\
\hline 79 & 0,0 & 0,862 & $-17,9$ & 1560,0 & 99,8 & 7,524 \\
\hline 80 & 0,0 & 0,858 & $-18,1$ & 1580,0 & 96,4 & 7,417 \\
\hline 81 & 0,0 & 0,853 & $-18,3$ & 1600,0 & 92,8 & 7,309 \\
\hline 82 & 0,0 & 0,849 & $-18,5$ & 1620,0 & 89,2 & 7,196 \\
\hline 83 & 0,0 & 0,845 & $-18,7$ & 1640,0 & 85,5 & 7,075 \\
\hline 84 & 0,0 & 0,840 & $-18,9$ & 1660,0 & 81,8 & 6,952 \\
\hline 85 & 0,0 & 0,836 & $-19,1$ & 1680,0 & 77,9 & 6,818 \\
\hline 86 & 0,0 & 0,831 & $-19,3$ & 1700,0 & 73,9 & 6,682 \\
\hline 87 & 0,0 & 0,826 & $-19,5$ & 1720,0 & 69,9 & 6,538 \\
\hline 88 & 0,0 & 0,821 & $-19,7$ & 1740,0 & 65,8 & 6,381 \\
\hline 89 & 0,0 & 0,815 & $-20,0$ & 1760,0 & 61,5 & 6,219 \\
\hline 90 & 0,0 & 0,810 & $-20,2$ & 1780,0 & 57,1 & 6,040 \\
\hline 91 & 0,0 & 0,804 & $-20,5$ & 1800,0 & 52,6 & 5,854 \\
\hline 92 & 0,0 & 0,797 & $-20,8$ & 1820,0 & 48,0 & 5,652 \\
\hline 93 & 0,0 & 0,791 & $-21,1$ & 1840,0 & 43,2 & 5,425 \\
\hline 94 & 0,0 & 0,784 & $-21,4$ & 1860,0 & 38,3 & 5,182 \\
\hline 95 & 0,0 & 0,776 & $-21,7$ & 1880,0 & 33,4 & 4,902 \\
\hline 96 & 0,0 & 0,767 & $-22,1$ & 1900,0 & 28,2 & 4,593 \\
\hline 97 & 0,0 & 0,758 & $-22,5$ & 1920,0 & 23,0 & 4,235 \\
\hline 98 & 0,0 & 0,747 & $-22,9$ & 1940,0 & 17,4 & 3,791 \\
\hline 99 & 0,0 & 0,735 & $-23,5$ & 1960,0 & 11,9 & 3,242 \\
\hline 100 & 0,0 & 0,718 & $-24,2$ & 1980,0 & 6,1 & 2,429 \\
\hline 101 & 0,0 & 0,682 & $-25,6$ & 2000,0 & 0,1 & 0,419 \\
\hline 102 & 0,0 & 0,680 & $-25,7$ & 2000,0 & 0,0 & 0,268 \\
\hline 103 & 0,0 & 0,676 & $-25,9$ & 2001,0 & 0,0 & 1,523 \\
\hline 104 & 0,0 & 0,672 & $-26,0$ & 2000,0 & 0,0 & $-0,278$ \\
\hline 105 & 0,0 & 0,669 & $-26,2$ & 2000,0 & $-0,1$ & $-0,443$ \\
\hline 106 & 0,0 & 0,665 & $-26,3$ & 1999,0 & $-0,4$ & $-0,769$ \\
\hline 107 & 0,0 & 0,662 & $-26,4$ & 1998,0 & $-0,6$ & $-1,003$ \\
\hline 108 & 0,0 & 0,658 & $-26,6$ & 1997,0 & $-1,0$ & $-1,266$ \\
\hline 109 & 0,0 & 0,654 & $-26,7$ & 1996,0 & $-1,4$ & $-1,539$ \\
\hline 110 & 0,0 & 0,651 & $-26,8$ & 1994,0 & $-1,9$ & $-1,824$ \\
\hline 111 & 0,0 & 0,647 & $-27,0$ & 1992,0 & $-2,5$ & $-2,081$ \\
\hline
\end{tabular}




\begin{tabular}{|c|c|c|c|c|c|c|}
\hline Casos & Tipo & $\begin{array}{c}\mathrm{V}_{5} \\
\text { (p.u.) }\end{array}$ & $\begin{array}{l}\Theta_{5} \\
\left(^{\circ}\right)\end{array}$ & $\begin{array}{c}\Delta \\
\text { Carga }(\%)\end{array}$ & $M_{Q V}$ & $\left(\frac{\partial Q}{\partial V}\right)^{\prime \prime}$ \\
\hline 112 & 0,0 & 0,644 & $-27,1$ & 1990,0 & $-3,2$ & $-2,397$ \\
\hline 113 & 0,0 & 0,641 & $-27,2$ & 1987,0 & $-3,9$ & $-2,722$ \\
\hline 114 & 0,0 & 0,637 & $-27,4$ & 1985,0 & $-4,7$ & $-3,033$ \\
\hline 115 & 0,0 & 0,634 & $-27,5$ & 1982,0 & $-5,6$ & $-3,353$ \\
\hline 116 & 0,0 & 0,630 & $-27,6$ & 1979,0 & $-6,5$ & $-3,664$ \\
\hline 117 & 0,0 & 0,627 & $-27,7$ & 1975,0 & $-7,4$ & $-4,020$ \\
\hline 118 & 0,0 & 0,624 & $-27,8$ & 1972,0 & $-8,4$ & $-4,368$ \\
\hline 119 & 0,0 & 0,620 & $-27,9$ & 1968,0 & $-9,6$ & $-4,734$ \\
\hline 120 & 0,0 & 0,617 & $-28,1$ & 1964,0 & $-10,7$ & $-5,116$ \\
\hline 121 & 0,0 & 0,614 & $-28,2$ & 1960,0 & $-11,8$ & $-5,491$ \\
\hline 122 & 0,0 & 0,611 & $-28,3$ & 1956,0 & $-13,0$ & $-5,861$ \\
\hline 123 & 0,0 & 0,608 & $-28,4$ & 1951,0 & $-14,3$ & $-6,279$ \\
\hline 124 & 0,0 & 0,604 & $-28,5$ & 1947,0 & $-15,6$ & $-6,670$ \\
\hline 125 & 0,0 & 0,601 & $-28,6$ & 1942,0 & $-16,9$ & $-7,106$ \\
\hline 126 & 0,0 & 0,598 & $-28,7$ & 1937,0 & $-18,2$ & $-7,517$ \\
\hline 127 & 0,0 & 0,595 & $-28,8$ & 1932,0 & $-19,6$ & $-7,950$ \\
\hline 128 & 0,0 & 0,592 & $-28,9$ & 1927,0 & $-21,1$ & $-8,413$ \\
\hline 129 & 0,0 & 0,589 & $-28,9$ & 1922,0 & $-22,5$ & $-8,872$ \\
\hline 130 & 0,0 & 0,586 & $-29,0$ & 1916,0 & $-23,9$ & $-9,362$ \\
\hline 131 & 0,0 & 0,583 & $-29,1$ & 1911,0 & $-25,4$ & $-9,855$ \\
\hline 132 & 0,0 & 0,581 & $-29,2$ & 1905,0 & $-26,8$ & $-10,352$ \\
\hline 133 & 0,0 & 0,578 & $-29,2$ & 1899,0 & $-28,4$ & $-10,883$ \\
\hline 134 & 0,0 & 0,575 & $-29,3$ & 1894,0 & $-29,9$ & $-11,416$ \\
\hline 135 & 0,0 & 0,572 & $-29,4$ & 1887,0 & $-31,4$ & $-11,986$ \\
\hline 136 & 0,0 & 0,570 & $-29,4$ & 1882,0 & $-32,9$ & $-12,535$ \\
\hline 137 & 0,0 & 0,567 & $-29,5$ & 1876,0 & $-34,4$ & $-13,125$ \\
\hline 138 & 0,0 & 0,564 & $-29,5$ & 1869,0 & $-36,0$ & $-13,726$ \\
\hline 139 & 0,0 & 0,562 & $-29,6$ & 1864,0 & $-37,6$ & $-14,308$ \\
\hline 140 & 0,0 & 0,559 & $-29,6$ & 1857,0 & $-39,1$ & $-14,965$ \\
\hline
\end{tabular}

Na Figura 5.5 observa-se o panorama geral do comportamento do índice $\left(\frac{\partial Q}{\partial V}\right)$ " versus o aumento de carga na barra 5. Pode-se notar a deterioração do índice $\left(\frac{\partial Q}{\partial V}\right)^{\prime \prime}$ com o aumento dos patamares de carga na barra 2 e as descontinuidades que ocorrem quando da perda de controle de tensão nas barras do tipo PV. 


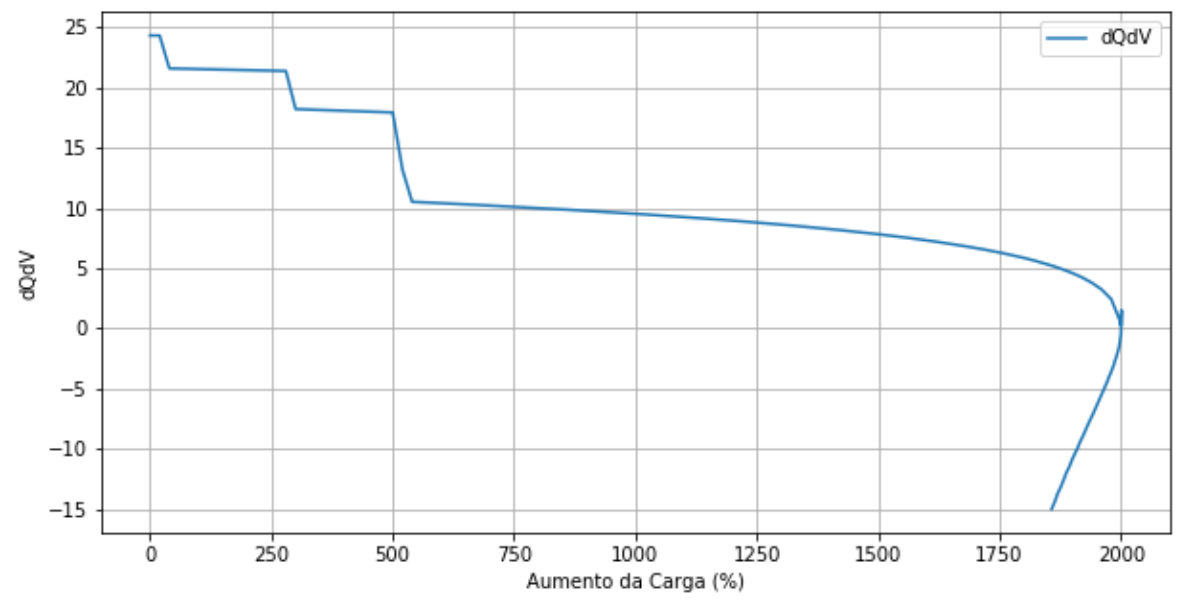

Figura 5.5: Índice $\left(\frac{\partial Q}{\partial V}\right) "$ na Barra 5

\subsubsection{5}

\section{Conclusão}

Para a barra 1 existem regiões de operação em que o índice de margem da curva QV é nulo, mas o índice $\left(\frac{\partial Q}{\partial V}\right)$ " não. Essa divergência de resultados nos índices $M_{Q V}$ e $\left(\frac{\partial Q}{\partial V}\right)$ " ocorre entre os Casos 25 ao 30, entre os Casos 102 ao 123, já de pouco interesse prático.

Nas demais barras analisadas (2, 4 e 5) não foi detectada essa divergência de resultados entre os índices.

\section{2 .2}

\section{Estudos com Crescimento de Carga e Geração em Múltiplas Barras}

Nessa Seção é utilizado, mais uma vez, o sistema de teste IEEE 14 barras [16] para avaliação dos índices de estabilidade de tensão.

Porém, agora o aumento de carga acorre nas barras 4 e 5, enquanto o gerador responsável por acompanhar o aumento de carga é o da barra 2 . A barra 1 é barra swing do sistema, responsável por absorver as perdas de potência ativa. Com o aumento de carga em múltiplas barras espera-se atingir o lado esquerdo da curva QV em um menor número de pontos de operação.

O FPC do programa ANAREDE é utilizado, assim como na Seção 5.2.1. Nesse estudo são gerados 124 casos com diferentes patamares de carga.

Os índices de sensibilidade $\left(\frac{\partial Q}{\partial V}\right)$ " e a margem da curva QV também serão analisados para cada patamar de carga do FPC nas barras 1, 2, 4 e 5 nas próximas seções. E conforme definido na Seção 4.3, a margem da curva QV informa a distância em MW do ponto de máximo carregamento. 


\subsubsection{1}

\section{Análise da Barra 1}

A análise da barra 1 tem como objetivo mostrar o comportamento do índice $\left(\frac{\partial Q}{\partial V}\right)$ ", a margem e o lado da curva QV após sucessivos aumentos de 20 $\%$ no patamar de carga nas barras 4 e 5.

Na Tabela 5.6 apresentam-se potência ativa e reativa gerada, tensão, ângulo, tipo da barra, e aumento percentual da carga. O índice $\left(\frac{\partial Q}{\partial V}\right)$ " e margem da curva QV $\left(M_{Q V}\right)$ também são apresentados na Tabela 5.6.

A barra 1 é a barra de referência do sistema e, portanto, $\mathrm{V}$ e $\Theta$ são constantes nessa barra. Essa barra absorve as perdas de potência ativa e, junto com as barras PV, as perdas reativa do sistema.

O Caso 1 corresponde ao caso-base do sistema e possui potência ativa na barra 1 igual a 232,4 MW e tensão de 1,06 p.u. A $M_{Q V}$ não é calculada pelo FPC do programa ANAREDE no Caso 1.

Tabela 5.6: Análise na Barra 1 - Crescimento de Carga nas Barras 4 e 5

\begin{tabular}{|c|c|c|c|c|c|c|c|c|}
\hline Casos & Tipo & $\begin{array}{c}\mathbf{P G}_{1} \\
(\mathrm{MW})\end{array}$ & $\begin{array}{c}\mathrm{QG}_{1} \\
(\mathrm{Mvar})\end{array}$ & $\begin{array}{c}\mathrm{V}_{1} \\
\text { (p.u.) }\end{array}$ & $\begin{array}{l}\Theta_{1} \\
\left({ }^{\circ}\right)\end{array}$ & $\begin{array}{c}\Delta \\
\text { Carga }(\%)\end{array}$ & $M_{Q V}$ & $\left(\frac{\partial Q}{\partial V}\right)^{\prime \prime}$ \\
\hline 1 & 2,0 & 232,4 & $-16,5$ & 1,06 & 0,0 & 0,0 & $\mathrm{NaN}$ & 21,290 \\
\hline 2 & 2,0 & 233,0 & $-15,7$ & 1,06 & 0,0 & 20,0 & 30,9 & 21,295 \\
\hline 3 & 2,0 & 233,7 & $-14,9$ & 1,06 & $-0,0$ & 40,0 & 29,8 & 21,301 \\
\hline 4 & 2,0 & 234,4 & $-14,1$ & 1,06 & $-0,0$ & 60,0 & 28,1 & 21,305 \\
\hline 5 & 2,0 & 235,2 & $-13,2$ & 1,06 & $-0,0$ & 80,0 & 27,6 & 21,310 \\
\hline 6 & 2,0 & 236,1 & $-12,4$ & 1,06 & $-0,0$ & 100,0 & 26,6 & 21,315 \\
\hline 7 & 2,0 & 237,0 & $-11,5$ & 1,06 & $-0,0$ & 120,0 & 24,4 & 21,319 \\
\hline 8 & 2,0 & 237,9 & $-10,6$ & 1,06 & $-0,0$ & 140,0 & 22,9 & 21,324 \\
\hline 9 & 2,0 & 239,0 & $-9,7$ & 1,06 & $-0,0$ & 160,0 & 21,8 & 21,328 \\
\hline 10 & 2,0 & 240,0 & $-8,7$ & 1,06 & $-0,0$ & 180,0 & 19,2 & 21,332 \\
\hline 11 & 2,0 & 241,2 & $-7,8$ & 1,06 & $-0,0$ & 200,0 & 16,1 & 21,336 \\
\hline 12 & 2,0 & 242,4 & $-6,8$ & 1,06 & $-0,0$ & 220,0 & 15,2 & 21,340 \\
\hline 13 & 2,0 & 243,6 & $-5,8$ & 1,06 & $-0,0$ & 240,0 & 12,6 & 21,344 \\
\hline 14 & 2,0 & 245,0 & $-4,6$ & 1,06 & $-0,0$ & 260,0 & 9,1 & 21,019 \\
\hline 15 & 2,0 & 246,4 & $-3,2$ & 1,06 & $-0,0$ & 280,0 & 5,4 & 20,879 \\
\hline 16 & 2,0 & 247,9 & $-1,5$ & 1,06 & $-0,0$ & 300,0 & 1,7 & 20,884 \\
\hline 17 & 2,0 & 249,5 & 0,1 & 1,06 & $-0,0$ & 320,0 & 0,0 & 20,888 \\
\hline 18 & 2,0 & 251,1 & 4,3 & 1,06 & $-0,0$ & 340,0 & 0,0 & $-4,177$ \\
\hline 19 & 2,0 & 253,0 & 10,6 & 1,06 & $-0,0$ & 360,0 & 0,0 & $-4,454$ \\
\hline 20 & 2,0 & 255,0 & 17,3 & 1,06 & $-0,0$ & 380,0 & 0,0 & $-4,752$ \\
\hline 21 & 2,0 & 257,0 & 24,5 & 1,06 & $-0,0$ & 400,0 & 0,0 & $-5,091$ \\
\hline 22 & 2,0 & 259,3 & 32,2 & 1,06 & $-0,0$ & 420,0 & $-1,8$ & $-5,475$ \\
\hline 23 & 2,0 & 261,7 & 40,4 & 1,06 & $-0,0$ & 440,0 & $-6,0$ & $-5,909$ \\
\hline
\end{tabular}




\begin{tabular}{ccccccccc}
\hline Casos & Tipo & $\begin{array}{c}\mathbf{P G}_{1} \\
(\mathbf{M W})\end{array}$ & $\begin{array}{c}\mathbf{Q G}_{1} \\
(\mathbf{M v a r})\end{array}$ & $\begin{array}{c}\mathbf{V}_{1} \\
(\mathbf{p . u} .)\end{array}$ & $\begin{array}{c}\Theta_{1} \\
\mathbf{(}^{\circ}\end{array}$ & $\begin{array}{c}\Delta \\
\text { Carga (\%) }\end{array}$ & $M_{Q V}$ & $\left(\frac{\partial Q}{\partial V}\right)^{\prime \prime}$ \\
\hline 24 & 2,0 & 264,3 & 49,3 & 1,06 & $-0,0$ & 460,0 & $-6,5$ & $-6,426$ \\
\hline 25 & 2,0 & 267,0 & 59,0 & 1,06 & $-0,0$ & 480,0 & $-7,1$ & $-7,009$ \\
\hline 26 & 2,0 & 270,0 & 69,4 & 1,06 & $-0,0$ & 500,0 & $-9,4$ & $-7,703$ \\
\hline 27 & 2,0 & 273,3 & 80,8 & 1,06 & $-0,0$ & 520,0 & $-16,6$ & $-8,535$ \\
\hline 28 & 2,0 & 276,9 & 93,4 & 1,06 & $-0,0$ & 540,0 & $-18,5$ & $-9,541$ \\
\hline 29 & 2,0 & 280,9 & 107,4 & 1,06 & $-0,0$ & 560,0 & $-20,8$ & $-10,853$ \\
\hline 30 & 2,0 & 285,4 & 123,2 & 1,06 & $-0,0$ & 580,0 & $-32,1$ & $-12,507$ \\
\hline 31 & 2,0 & 290,5 & 141,3 & 1,06 & $-0,0$ & 600,0 & $-38,9$ & $-14,797$ \\
\hline 32 & 2,0 & 296,6 & 162,8 & 1,06 & $-0,0$ & 620,0 & $-46,0$ & $-18,198$ \\
\hline 33 & 2,0 & 304,1 & 189,6 & 1,06 & $-0,0$ & 640,0 & $-68,5$ & $-23,917$ \\
\hline 34 & 2,0 & 314,6 & 227,6 & 1,06 & $-0,0$ & 660,0 & $-91,6$ & $-38,064$ \\
\hline 35 & 2,0 & 323,1 & 258,3 & 1,06 & $-0,0$ & 670,0 & $-113,4$ & $-60,617$ \\
\hline 36 & 2,0 & 337,1 & 299,3 & 1,06 & $-0,0$ & 675,0 & $-151,2$ & $-202,858$ \\
\hline 37 & 2,0 & 338,7 & 305,0 & 1,06 & $-0,0$ & 675,0 & $-156,9$ & $-202,871$ \\
\hline 38 & 2,0 & 339,2 & 306,9 & 1,06 & $-0,0$ & 675,0 & 0,0 & $-202,844$ \\
\hline 39 & 2,0 & 340,7 & 312,4 & 1,06 & $-0,0$ & 675,0 & 0,0 & $-413,371$ \\
\hline 40 & 2,0 & 342,2 & 317,9 & 1,06 & $-0,0$ & 675,0 & 0,0 & $-413,307$ \\
\hline 41 & 2,0 & 343,5 & 323,4 & 1,06 & $-0,0$ & 675,0 & 0,0 & $-862,080$ \\
\hline
\end{tabular}

O índice $\left(\frac{\partial Q}{\partial V}\right)$ " apresenta descontinuidade quando ocorre perda de controle de tensão, ou seja, quando uma determinada barra atinge o seu limite de geração de potência reativa. Isso ocorre no Caso 14 para as barras 4 e 6, Caso 15 para a barra 3, Caso 18 para barra 2.

Na Tabela 5.7 apresenta-se o tipo de barra, da barra 1 à barra 8. Pode-se observar a perda de controle de tensão que ocorre nas barras do tipo PV.

Ao comparar a perda de continuidade nos índices $\left(\frac{\partial Q}{\partial V}\right)$ " obtidos nessa seção com o ocorrido na Seção 5.2.1, percebe-se que aqui as barras 6 e 8 são as que primeiro sofrem com a perda de continuidade. Isso ocorre, pois, essas barras são as mais próximas das barras 4 e 5 , onde ocorre o aumento de carga.

O índice da barra 1 passa por zero entre os Casos 17 e 18, pois neste caso deixam de existir barras com controle de tensão, como já explicado na Seção 5.2.1.

O comportamento geral do índice $M_{Q V}$ indica sua diminuição com o aumento da carga do sistema, balisando sua aproximação ao lado esquerdo da curva QV. A partir do Caso 17 os índices $M_{Q V}$ e $\left(\frac{\partial Q}{\partial V}\right)$ " apresentam valores abaixo de zero, atuando como indicação de que o SEE opera fora da região de operação normal.

O índice $M_{Q V}$ diminui com o aumento da carga, indicando a aproximação ao lado esquerdo da curva QV. 
Tabela 5.7: Tipos das Barras - Crescimento de Carga nas Barras 4 e 5

\begin{tabular}{ccccccccc}
\hline Casos & Barra & Barra & Barra & Barra & Barra & Barra & Barra & Barra \\
& $\mathbf{1}$ & $\mathbf{2}$ & $\mathbf{3}$ & $\mathbf{4}$ & $\mathbf{5}$ & $\mathbf{6}$ & $\mathbf{7}$ & $\mathbf{8}$ \\
\hline 1 & 2 & 1 & 1 & 0 & 0 & 1 & 0 & 1 \\
\hline 2 & 2 & 1 & 1 & 0 & 0 & 1 & 0 & 1 \\
\hline 3 & 2 & 1 & 1 & 0 & 0 & 1 & 0 & 1 \\
\hline 4 & 2 & 1 & 1 & 0 & 0 & 1 & 0 & 1 \\
\hline 5 & 2 & 1 & 1 & 0 & 0 & 1 & 0 & 1 \\
\hline 6 & 2 & 1 & 1 & 0 & 0 & 1 & 0 & 1 \\
\hline 7 & 2 & 1 & 1 & 0 & 0 & 1 & 0 & 1 \\
\hline 8 & 2 & 1 & 1 & 0 & 0 & 1 & 0 & 1 \\
\hline 9 & 2 & 1 & 1 & 0 & 0 & 1 & 0 & 1 \\
\hline 10 & 2 & 1 & 1 & 0 & 0 & 1 & 0 & 1 \\
\hline 11 & 2 & 1 & 1 & 0 & 0 & 1 & 0 & 1 \\
\hline 12 & 2 & 1 & 1 & 0 & 0 & 1 & 0 & 1 \\
\hline 13 & 2 & 1 & 1 & 0 & 0 & 1 & 0 & 1 \\
\hline 14 & 2 & 1 & 1 & 0 & 0 & -1 & 0 & -1 \\
\hline 15 & 2 & 1 & -1 & 0 & 0 & -1 & 0 & -1 \\
\hline 16 & 2 & 1 & -1 & 0 & 0 & -1 & 0 & -1 \\
\hline 17 & 2 & 1 & -1 & 0 & 0 & -1 & 0 & -1 \\
\hline 18 & 2 & -1 & -1 & 0 & 0 & -1 & 0 & -1 \\
\hline 19 & 2 & -1 & -1 & 0 & 0 & -1 & 0 & -1 \\
\hline 20 & 2 & -1 & -1 & 0 & 0 & -1 & 0 & -1 \\
\hline 21 & 2 & -1 & -1 & 0 & 0 & -1 & 0 & -1 \\
\hline 22 & 2 & -1 & -1 & 0 & 0 & -1 & 0 & -1 \\
\hline 23 & 2 & -1 & -1 & 0 & 0 & -1 & 0 & -1 \\
\hline 24 & 2 & -1 & -1 & 0 & 0 & -1 & 0 & -1 \\
\hline 25 & 2 & -1 & -1 & 0 & 0 & -1 & 0 & -1 \\
\hline 26 & 2 & -1 & -1 & 0 & 0 & -1 & 0 & -1 \\
\hline 27 & 2 & -1 & -1 & 0 & 0 & -1 & 0 & -1 \\
\hline 28 & 2 & -1 & -1 & 0 & 0 & -1 & 0 & -1 \\
\hline 29 & 2 & -1 & -1 & 0 & 0 & -1 & 0 & -1 \\
\hline 30 & 2 & -1 & -1 & 0 & 0 & -1 & 0 & -1 \\
\hline 31 & 2 & -1 & -1 & 0 & 0 & -1 & 0 & -1 \\
\hline 32 & 2 & -1 & -1 & 0 & 0 & -1 & 0 & -1 \\
\hline 33 & 2 & -1 & -1 & 0 & 0 & -1 & 0 & -1 \\
\hline 34 & 2 & -1 & -1 & 0 & 0 & -1 & 0 & -1 \\
\hline 35 & 2 & -1 & -1 & 0 & 0 & -1 & 0 & -1 \\
\hline 36 & 2 & -1 & -1 & 0 & 0 & -1 & 0 & -1 \\
\hline 37 & 2 & -1 & -1 & 0 & 0 & -1 & 0 & -1 \\
\hline 38 & 2 & -1 & -1 & 0 & 0 & -1 & 0 & -1 \\
\hline 39 & 2 & -1 & -1 & 0 & 0 & -1 & 0 & -1 \\
\hline & & & & & & & & \\
\hline
\end{tabular}


A carga máxima ocorre nos Casos 36 a 40 quando ambos os índices são negativos. A partir daí o interesse prático é pequeno.

Na Figura 5.6 observa-se o panorama geral do comportamento do índice $\left(\frac{\partial Q}{\partial V}\right)$ " versus o aumento de carga nas barras 4 e 5 . Pode-se notar a deterioração do índice $\left(\frac{\partial Q}{\partial V}\right)$ " com o aumento dos patamares de carga e as descontinuidades que ocorrem quando da perda de tensão controlada nas barras do tipo PV.

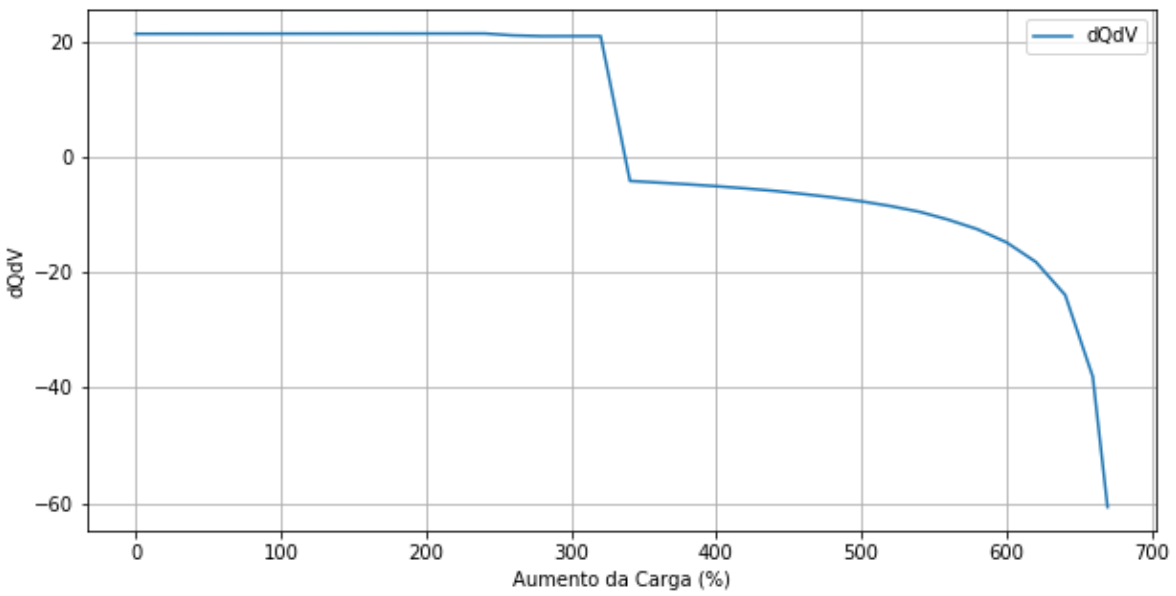

Figura 5.6: Índice $\left(\frac{\partial Q}{\partial V}\right) "$ na Barra 1

\subsubsection{2}

\section{Análise da Barra 2}

A análise da barra 2 tem como objetivo mostrar o comportamento do índice $\left(\frac{\partial Q}{\partial V}\right)$ ", a margem e o lado da curva QV após sucessivos aumentos de 20 $\%$ no patamar de carga nas barras 4 e 5 .

Na Tabela 5.8 apresentam-se potência ativa e reativa gerada, tensão, ângulo, tipo da barra, e aumento percentual da carga. O índice $\left(\frac{\partial Q}{\partial V}\right)$ " e margem da curva QV $\left(M_{Q V}\right)$ também são apresentados na Tabela 5.8.

A barra 2 é configurada através do comando do comando DGER do ANAREDE para compensar o aumento da demanda de potência ativa nas barras 4 e 5 .

O Caso 1 corresponde ao caso-base, possuindo potência ativa na barra 2 igual a 40,0 MW, potência reativa de 43,6 Mvar, e tensão de 1,045 p.u.. 
Tabela 5.8: Análise na Barra 2 - Crescimento de Carga nas Barras 4 e 5

\begin{tabular}{|c|c|c|c|c|c|c|c|c|}
\hline Casos & Tipo & $\begin{array}{c}\mathbf{P G}_{2} \\
(\mathrm{MW})\end{array}$ & $\begin{array}{c}\mathrm{QG}_{2} \\
\text { (Mvar) }\end{array}$ & $\begin{array}{c}\mathrm{V}_{2} \\
\text { (p.u.) }\end{array}$ & $\begin{array}{l}\Theta_{2} \\
\left(^{\circ}\right)\end{array}$ & $\begin{array}{c}\Delta \\
\text { Carga (\%) }\end{array}$ & $M_{Q V}$ & $\left(\frac{\partial Q}{\partial V}\right)^{\prime}$ \\
\hline 1 & 1,0 & 40,0 & 43,6 & 1,045 & $-5,0$ & 0,0 & 484,5 & 30,200 \\
\hline 2 & 1,0 & 51,1 & 42,6 & 1,045 & $-4,9$ & 20,0 & 477,9 & 30,166 \\
\hline 3 & 1,0 & 62,2 & 41,7 & 1,045 & $-4,9$ & 40,0 & 471,2 & 30,131 \\
\hline 4 & 1,0 & 73,2 & 41,0 & 1,045 & $-4,8$ & 60,0 & 463,9 & 30,096 \\
\hline 5 & 1,0 & 84,3 & 40,3 & 1,045 & $-4,8$ & 80,0 & 456,1 & 30,059 \\
\hline 6 & 1,0 & 95,4 & 39,8 & 1,045 & $-4,7$ & 100,0 & 448,0 & 30,021 \\
\hline 7 & 1,0 & 106,5 & 39,3 & 1,045 & $-4,7$ & 120,0 & 439,4 & 29,982 \\
\hline 8 & 1,0 & 117,6 & 39,0 & 1,045 & $-4,6$ & 140,0 & 430,3 & 29,942 \\
\hline 9 & 1,0 & 128,6 & 38,8 & 1,045 & $-4,6$ & 160,0 & 420,8 & 29,901 \\
\hline 10 & 1,0 & 139,7 & 38,7 & 1,045 & $-4,6$ & 180,0 & 410,8 & 29,858 \\
\hline 11 & 1,0 & 150,8 & 38,7 & 1,045 & $-4,5$ & 200,0 & 400,4 & 29,815 \\
\hline 12 & 1,0 & 161,9 & 38,8 & 1,045 & $-4,5$ & 220,0 & 389,5 & 29,769 \\
\hline 13 & 1,0 & 173,0 & 39,0 & 1,045 & $-4,4$ & 240,0 & 378,1 & 29,723 \\
\hline 14 & 1,0 & 184,0 & 39,9 & 1,045 & $-4,4$ & 260,0 & 366,7 & 27,572 \\
\hline 15 & 1,0 & 195,1 & 41,7 & 1,045 & $-4,4$ & 280,0 & 355,8 & 19,258 \\
\hline 16 & 1,0 & 206,2 & 45,0 & 1,045 & $-4,3$ & 300,0 & 345,6 & 19,161 \\
\hline 17 & 1,0 & 217,3 & 48,4 & 1,045 & $-4,3$ & 320,0 & 335,1 & 19,058 \\
\hline 18 & $-1,0$ & 228,4 & 50,0 & 1,044 & $-4,3$ & 340,0 & 322,1 & 18,900 \\
\hline 19 & $-1,0$ & 239,4 & 50,0 & 1,042 & $-4,2$ & 360,0 & 307,0 & 18,676 \\
\hline 20 & $-1,0$ & 250,5 & 50,0 & 1,040 & $-4,2$ & 380,0 & 291,1 & 18,444 \\
\hline 21 & $-1,0$ & 261,6 & 50,0 & 1,037 & $-4,1$ & 400,0 & 275,1 & 18,188 \\
\hline 22 & $-1,0$ & 272,7 & 50,0 & 1,034 & $-4,0$ & 420,0 & 258,4 & 17,909 \\
\hline 23 & $-1,0$ & 283,8 & 50,0 & 1,032 & $-4,0$ & 440,0 & 240,9 & 17,607 \\
\hline 24 & $-1,0$ & 294,8 & 50,0 & 1,028 & $-3,9$ & 460,0 & 223,2 & 17,260 \\
\hline 25 & $-1,0$ & 305,9 & 50,0 & 1,025 & $-3,9$ & 480,0 & 204,9 & 16,891 \\
\hline 26 & $-1,0$ & 317,0 & 50,0 & 1,021 & $-3,8$ & 500,0 & 186,0 & 16,475 \\
\hline 27 & $-1,0$ & 328,1 & 50,0 & 1,017 & $-3,8$ & 520,0 & 166,8 & 16,006 \\
\hline 28 & $-1,0$ & 339,2 & 50,0 & 1,012 & $-3,7$ & 540,0 & 146,8 & 15,478 \\
\hline 29 & $-1,0$ & 350,2 & 50,0 & 1,007 & $-3,7$ & 560,0 & 126,7 & 14,841 \\
\hline 30 & $-1,0$ & 361,3 & 50,0 & 1,001 & $-3,6$ & 580,0 & 105,9 & 14,117 \\
\hline 31 & $-1,0$ & 372,4 & 50,0 & 0,995 & $-3,5$ & 600,0 & 84,4 & 13,226 \\
\hline 32 & $-1,0$ & 383,5 & 50,0 & 0,987 & $-3,5$ & 620,0 & 62,8 & 12,090 \\
\hline 33 & $-1,0$ & 394,6 & 50,0 & 0,976 & $-3,4$ & 640,0 & 40,5 & 10,546 \\
\hline 34 & $-1,0$ & 405,6 & 50,0 & 0,962 & $-3,3$ & 660,0 & 18,3 & 7,904 \\
\hline 35 & $-1,0$ & 411,2 & 50,0 & 0,950 & $-3,3$ & 670,0 & 6,8 & 5,418 \\
\hline 36 & $-1,0$ & 411,2 & 50,0 & 0,933 & $-3,4$ & 675,0 & 0,0 & 0,716 \\
\hline 37 & $-1,0$ & 411,2 & 50,0 & 0,931 & $-3,4$ & 675,0 & 0,0 & 0,716 \\
\hline 38 & $-1,0$ & 411,2 & 50,0 & 0,930 & $-3,4$ & 675,0 & 0,0 & 0,717 \\
\hline 39 & $-1,0$ & 411,2 & 50,0 & 0,928 & $-3,4$ & 675,0 & 0,0 & $-0,756$ \\
\hline 40 & $-1,0$ & 411,2 & 50,0 & 0,926 & $-3,4$ & 675,0 & 0,0 & $-0,756$ \\
\hline 41 & $-1,0$ & 411,2 & 50,0 & 0,924 & $-3,4$ & 675,0 & $-0,2$ & $-1,589$ \\
\hline
\end{tabular}


O índice $M_{Q V}$ diminui com o aumento da carga, indicando a aproximação ao lado esquerdo da curva QV. A partir do Caso 41 os índices $M_{Q V}$ e $M_{Q V}$ e $\left(\frac{\partial Q}{\partial V}\right)^{\prime \prime}$ passam a apresentar valores negativos, indicando operação fora da região normal.

Na barra 2 também é possível perceber a perda de continuidade do índice $\left(\frac{\partial Q}{\partial V}\right)$ " nos Casos 14, 15 e 18 quando ocorre perda de tensão controlada das barras do tipo PV.

Os Casos 36 a 41 representam a carga máxima e até o Caso 35 ambos os índices são positivos como esperado. No Caso 36 o índice $M_{Q V}$ fica nulo e entre os Casos 38 e 39 o índice $\left(\frac{\partial Q}{\partial V}\right)$ " troca de sinal ficando negativo. A partir daí o interesse prático é pequeno.

Na Figura 5.7 observa-se o panorama geral do comportamento do índice $\left(\frac{\partial Q}{\partial V}\right)$ "versus o aumento de carga nas barras 4 e 5 . Pode-se notar a deterioração do índice $\left(\frac{\partial Q}{\partial V}\right)$ " com o aumento dos patamares de carga e as descontinuidades que ocorrem quando da perda de tensão controlada nas barras do tipo PV.

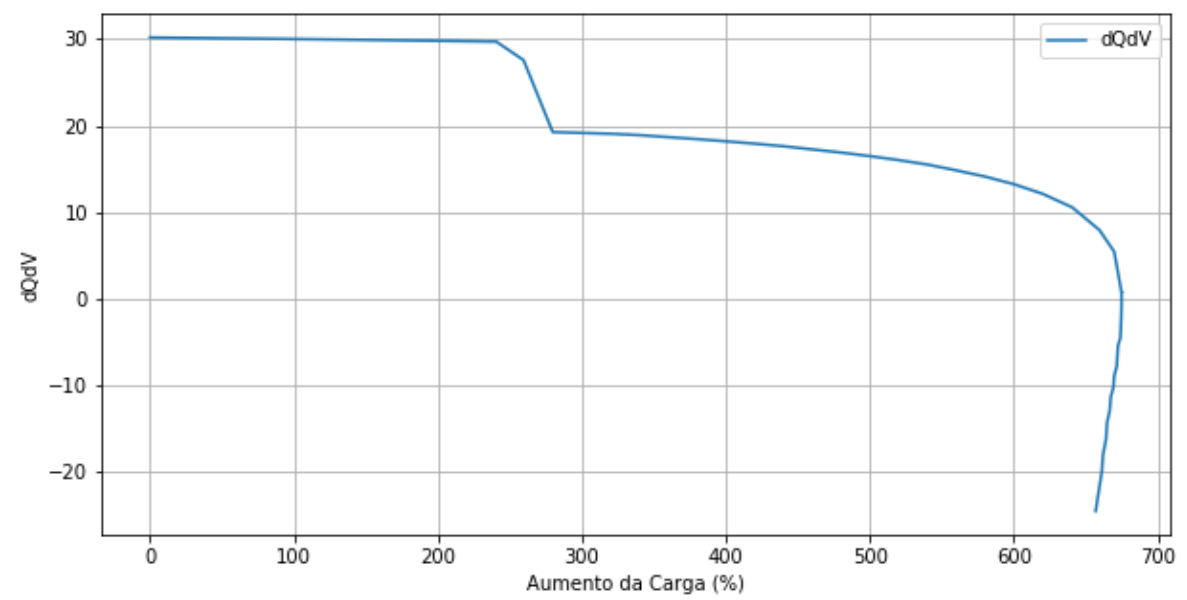

Figura 5.7: índice $\left(\frac{\partial Q}{\partial V}\right) "$ na Barra 2

\subsubsection{3}

\section{Análise da Barra 4}

A análise da barra 4 tem como objetivo mostrar o comportamento do índice $\left(\frac{\partial Q}{\partial V}\right)$ ", a margem e o lado da curva QV após sucessivos aumentos de 20 $\%$ no patamar de carga nas barras 4 e 5 .

Na Tabela 5.9 apresentam-se potência ativa e reativa de carga, tensão, ângulo, tipo da barra, e aumento percentual da carga. O índice $\left(\frac{\partial Q}{\partial V}\right)^{\prime \prime}$ e margem da curva QV $\left(M_{Q V}\right)$ também são apresentados na Tabela 5.9. 
A barra 4 é configurada para realizar o aumento de carga do sistema em patamares de $20 \%$. O Caso 1 tem $\mathrm{PL}=47,8 \mathrm{MW}, \mathrm{QL}=-3,9$ Mvar e tensão de 1,018 p.u. Esses patamares de carga serão elevados até atingir a carga máxima.

Tabela 5.9: Análise na Barra 4 - Crescimento de Carga nas Barras 4 e 5

\begin{tabular}{|c|c|c|c|c|c|c|c|c|}
\hline Casos & Tipo & $\begin{array}{c}\mathbf{P L}_{4} \\
(\mathrm{MW})\end{array}$ & $\begin{array}{c}\mathrm{QL}_{4} \\
\text { (Mvar) }\end{array}$ & $\begin{array}{c}\mathrm{V}_{4} \\
\text { (p.u.) }\end{array}$ & $\begin{array}{l}\Theta_{4} \\
\left({ }^{\circ}\right) \\
\end{array}$ & $\begin{array}{c}\Delta \\
\text { Carga (\%) }\end{array}$ & $M_{Q V}$ & $\left(\frac{\partial Q}{\partial V}\right)^{\prime}$ \\
\hline 1 & 0,0 & 47,8 & $-3,9$ & 1,018 & $-10,3$ & 0,0 & 238,0 & 24,836 \\
\hline 2 & 0,0 & 57,4 & $-4,7$ & 1,016 & $-10,7$ & 20,0 & 235,1 & 24,793 \\
\hline 3 & 0,0 & 66,9 & $-5,5$ & 1,015 & $-11,0$ & 40,0 & 231,9 & 24,749 \\
\hline 4 & 0,0 & 76,5 & $-6,2$ & 1,013 & $-11,4$ & 60,0 & 228,6 & 24,699 \\
\hline 5 & 0,0 & 86,0 & $-7,0$ & 1,011 & $-11,8$ & 80,0 & 225,1 & 24,651 \\
\hline 6 & 0,0 & 95,6 & $-7,8$ & 1,010 & $-12,1$ & 100,0 & 221,3 & 24,600 \\
\hline 7 & 0,0 & 105,2 & $-8,6$ & 1,008 & $-12,5$ & 120,0 & 217,3 & 24,547 \\
\hline 8 & 0,0 & 114,7 & $-9,4$ & 1,006 & $-12,9$ & 140,0 & 213,1 & 24,492 \\
\hline 9 & 0,0 & 124,3 & $-10,1$ & 1,005 & $-13,2$ & 160,0 & 208,6 & 24,431 \\
\hline 10 & 0,0 & 133,8 & $-10,9$ & 1,003 & $-13,6$ & 180,0 & 203,9 & 24,372 \\
\hline 11 & 0,0 & 143,4 & $-11,7$ & 1,001 & $-14,0$ & 200,0 & 199,0 & 24,310 \\
\hline 12 & 0,0 & 153,0 & $-12,5$ & 0,999 & $-14,4$ & 220,0 & 193,8 & 24,245 \\
\hline 13 & 0,0 & 162,5 & $-13,3$ & 0,997 & $-14,8$ & 240,0 & 188,4 & 24,179 \\
\hline 14 & 0,0 & 172,1 & $-14,0$ & 0,995 & $-15,2$ & 260,0 & 182,7 & 17,912 \\
\hline 15 & 0,0 & 181,6 & $-14,8$ & 0,992 & $-15,6$ & 280,0 & 176,8 & 14,647 \\
\hline 16 & 0,0 & 191,2 & $-15,6$ & 0,988 & $-16,0$ & 300,0 & 170,6 & 14,550 \\
\hline 17 & 0,0 & 200,8 & $-16,4$ & 0,985 & $-16,4$ & 320,0 & 164,2 & 14,449 \\
\hline 18 & 0,0 & 210,3 & $-17,2$ & 0,980 & $-16,8$ & 340,0 & 157,4 & 8,757 \\
\hline 19 & 0,0 & 219,9 & $-17,9$ & 0,974 & $-17,3$ & 360,0 & 150,4 & 8,593 \\
\hline 20 & 0,0 & 229,4 & $-18,7$ & 0,968 & $-17,8$ & 380,0 & 143,1 & 8,427 \\
\hline 21 & 0,0 & 239,0 & $-19,5$ & 0,962 & $-18,2$ & 400,0 & 135,6 & 8,246 \\
\hline 22 & 0,0 & 248,6 & $-20,3$ & 0,955 & $-18,7$ & 420,0 & 127,8 & 8,052 \\
\hline 23 & 0,0 & 258,1 & $-21,1$ & 0,947 & $-19,3$ & 440,0 & 119,6 & 7,845 \\
\hline 24 & 0,0 & 267,7 & $-21,8$ & 0,940 & $-19,8$ & 460,0 & 111,2 & 7,610 \\
\hline 25 & 0,0 & 277,2 & $-22,6$ & 0,931 & $-20,4$ & 480,0 & 102,4 & 7,367 \\
\hline 26 & 0,0 & 286,8 & $-23,4$ & 0,923 & $-21,0$ & 500,0 & 93,4 & 7,097 \\
\hline 27 & 0,0 & 296,4 & $-24,2$ & 0,913 & $-21,6$ & 520,0 & 84,0 & 6,800 \\
\hline 28 & 0,0 & 305,9 & $-25,0$ & 0,902 & $-22,3$ & 540,0 & 74,4 & 6,473 \\
\hline 29 & 0,0 & 315,5 & $-25,7$ & 0,891 & $-23,1$ & 560,0 & 64,4 & 6,091 \\
\hline 30 & 0,0 & 325,0 & $-26,5$ & 0,878 & $\begin{array}{l}-23,9 \\
\end{array}$ & 580,0 & 54,1 & 5,670 \\
\hline 31 & 0,0 & 334,6 & $-27,3$ & 0,863 & $-24,8$ & 600,0 & 43,5 & 5,174 \\
\hline 32 & 0,0 & 344,2 & $-28,1$ & 0,846 & $-25,9$ & 620,0 & 32,5 & 4,573 \\
\hline 33 & 0,0 & 353,7 & $-28,9$ & 0,825 & $-27,2$ & 640,0 & 21,2 & 3,811 \\
\hline 34 & 0,0 & 363,3 & $-29,6$ & 0,795 & $-28,9$ & 660,0 & 9,6 & 2,644 \\
\hline 35 & 0,0 & 368,1 & $-30,0$ & 0,771 & $-30,3$ & 670,0 & 3,6 & 1,688 \\
\hline 36 & 0,0 & 370,5 & $-30,2$ & 0,740 & $-32,2$ & 675,0 & 0,1 & 0,196 \\
\hline 37 & 0,0 & 370,5 & $-30,2$ & 0,735 & $-32,4$ & 675,0 & 0,0 & 0,196 \\
\hline
\end{tabular}




\begin{tabular}{ccccccccc}
\hline Casos & Tipo & $\begin{array}{c}\mathbf{P L}_{4} \\
(\mathbf{M W})\end{array}$ & $\begin{array}{c}\mathbf{Q L}_{4} \\
(\mathbf{M v a r})\end{array}$ & $\begin{array}{c}\mathbf{V}_{4} \\
(\mathbf{p . u .})\end{array}$ & $\begin{array}{c}\Theta_{4} \\
\left(^{\circ}\right.\end{array}$ & $\begin{array}{c}\Delta \\
\text { Carga (\%) }\end{array}$ & $M_{Q V}$ & $\left(\frac{\partial Q}{\partial V}\right)^{\prime \prime}$ \\
\hline 38 & 0,0 & 370,5 & $-30,2$ & 0,734 & $-32,5$ & 675,0 & 0,0 & 0,196 \\
\hline 39 & 0,0 & 370,5 & $-30,2$ & 0,729 & $-32,8$ & 675,0 & 0,0 & $-0,199$ \\
\hline 40 & 0,0 & 370,5 & $-30,2$ & 0,725 & $-33,0$ & 675,0 & $-0,1$ & $-0,199$ \\
\hline 41 & 0,0 & 370,4 & $-30,2$ & 0,721 & $-33,2$ & 675,0 & $-0,3$ & $-0,410$ \\
\hline
\end{tabular}

O índice $M_{Q V}$ diminui com o aumento da carga, indicando a aproximação ao lado esquerdo da curva QV. A partir do Caso 40 os índices $M_{Q V}$ e $\left(\frac{\partial Q}{\partial V}\right)$ " passam a apresentar valores negativos, indicando operação fora da região normal.

Após o Caso 40 o índice $M_{Q V}$ continua diminuindo com o aumento da carga demandada pelo Sistema nas barras 4 e 5. A análise da barra 4 não sinaliza um retorno a zero do índice $M_{Q V}$, portanto, não sinalizando a inversão da sensibilidade tensão/potência nessa barra.

A simulação de FPC diminui a carga até o Caso 86, quando o aumento de carga volta a ser positivo, ocorrendo o aumento da $M_{Q V}$. No Caso 111 ocorre uma nova carga máxima.

Na barra 4 também é possível perceber a perda de continuidade do índice $\left(\frac{\partial Q}{\partial V}\right)^{\prime}$ nos Casos 14, 15 e 18 quando ocorre perda de tensão controlada das barras do tipo PV.

Os Casos 36 a 41 representam a carga máxima e até o Caso 35 ambos os índices são positivos como esperado. No Caso 37 o índice $M_{Q V}$ fica nulo e entre os Casos 38 e 39 o índice $\left(\frac{\partial Q}{\partial V}\right)$ " troca de sinal ficando negativo. A partir daí o interesse prático é pequeno.

Na Figura 5.8 observa-se o panorama geral do comportamento do índice $\left(\frac{\partial Q}{\partial V}\right)^{\prime}$ versus o aumento de carga nas barras 4 e 5 . Pode-se notar a deterioração do índice $\left(\frac{\partial Q}{\partial V}\right)$ " com o aumento dos patamares de carga e as descontinuidades que ocorrem quando da perda de tensão controlada nas barras do tipo PV. 


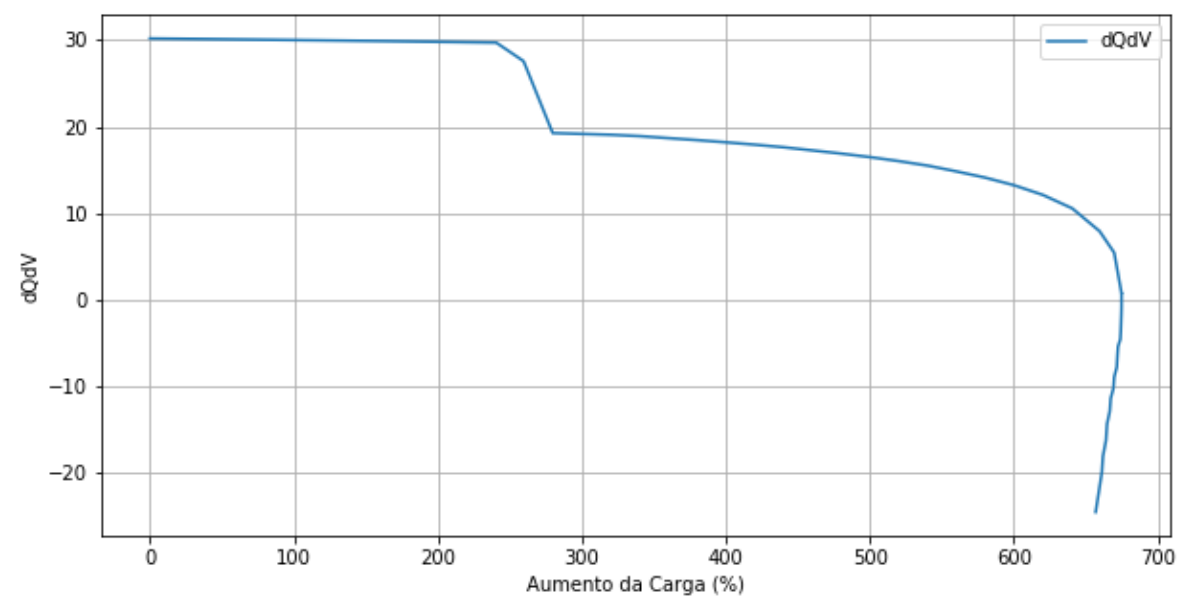

Figura 5.8: índice $\left(\frac{\partial Q}{\partial V}\right) "$ na Barra 4

\subsubsection{4}

\section{Análise da Barra 5}

A análise da barra 5 tem como objetivo mostrar o comportamento do índice $\left(\frac{\partial Q}{\partial V}\right)$ ", a margem e o lado da curva QV após sucessivos aumentos de 20 $\%$ no patamar de carga nas barras 4 e 5 .

Na Tabela 5.10 apresentam-se potência ativa e reativa de carga, tensão, ângulo, tipo da barra, e aumento percentual da carga. O índice $\left(\frac{\partial Q}{\partial V}\right)^{\prime \prime}$ e margem da curva QV $\left(M_{Q V}\right)$ também são apresentados na Tabela 5.10.

A barra 5 é configurada para realizar o aumento de carga do sistema em patamares de $20 \%$. O Caso 1 tem $\mathrm{PL}=7,6 \mathrm{MW}, \mathrm{QL}=1,6$ Mvar e tensão de 1,02 p.u.. Esses patamares de carga serão elevados até atingir a carga máxima.

Tabela 5.10: Análise na Barra 5 - Crescimento de Carga nas Barras 4 e 5

\begin{tabular}{ccccccccc}
\hline Casos & Tipo & $\begin{array}{c}\mathbf{P L}_{5} \\
(\mathbf{M W})\end{array}$ & $\begin{array}{c}\mathbf{Q L}_{5} \\
(\mathbf{M v a r})\end{array}$ & $\begin{array}{c}\mathbf{V}_{5} \\
(\mathbf{p . u .})\end{array}$ & $\begin{array}{c}\Theta_{5} \\
\left({ }^{\circ}\right)\end{array}$ & $\begin{array}{c}\Delta \\
\text { Carga (\%) }\end{array}$ & $M_{Q V}$ & $\left(\frac{\partial Q}{\partial V}\right)^{\prime \prime}$ \\
\hline 1 & 0,0 & 7,6 & 1,6 & 1,020 & $-8,8$ & 0,0 & 259,4 & 24,294 \\
\hline 2 & 0,0 & 9,1 & 1,9 & 1,018 & $-9,0$ & 20,0 & 255,9 & 24,247 \\
\hline 3 & 0,0 & 10,6 & 2,2 & 1,017 & $-9,3$ & 40,0 & 252,2 & 24,199 \\
\hline 4 & 0,0 & 12,2 & 2,6 & 1,015 & $-9,6$ & 60,0 & 248,3 & 24,144 \\
\hline 5 & 0,0 & 13,7 & 2,9 & 1,014 & $-9,9$ & 80,0 & 244,1 & 24,092 \\
\hline 6 & 0,0 & 15,2 & 3,2 & 1,013 & $-10,1$ & 100,0 & 239,8 & 24,038 \\
\hline 7 & 0,0 & 16,7 & 3,5 & 1,011 & $-10,4$ & 120,0 & 235,1 & 23,981 \\
\hline 8 & 0,0 & 18,2 & 3,8 & 1,010 & $-10,7$ & 140,0 & 230,3 & 23,923 \\
\hline 9 & 0,0 & 19,8 & 4,2 & 1,008 & $-11,0$ & 160,0 & 225,3 & 23,859 \\
\hline 10 & 0,0 & 21,3 & 4,5 & 1,006 & $-11,3$ & 180,0 & 220,0 & 23,797 \\
\hline
\end{tabular}




\begin{tabular}{ccccccccc}
\hline Casos & Tipo & $\begin{array}{c}\mathbf{P L}_{5} \\
(\mathbf{M W})\end{array}$ & $\begin{array}{c}\mathbf{Q L}_{5} \\
(\mathbf{M v a r})\end{array}$ & $\begin{array}{c}\mathbf{V}_{5} \\
(\mathbf{p . u .})\end{array}$ & $\begin{array}{c}\Theta_{5} \\
\mathbf{(}^{\circ}\end{array}$ & $\begin{array}{c}\Delta \\
\text { Carga }(\mathbf{\%})\end{array}$ & $M_{Q V}$ & $\left(\frac{\partial Q}{\partial V}\right)^{\prime}$ \\
\hline 11 & 0,0 & 22,8 & 4,8 & 1,005 & $-11,5$ & 200,0 & 214,4 & 23,731 \\
\hline 12 & 0,0 & 24,3 & 5,1 & 1,003 & $-11,8$ & 220,0 & 208,6 & 23,664 \\
\hline 13 & 0,0 & 25,8 & 5,4 & 1,002 & $-12,1$ & 240,0 & 202,6 & 23,595 \\
\hline 14 & 0,0 & 27,4 & 5,8 & 0,999 & $-12,4$ & 260,0 & 196,3 & 17,304 \\
\hline 15 & 0,0 & 28,9 & 6,1 & 0,997 & $-12,7$ & 280,0 & 189,7 & 15,490 \\
\hline 16 & 0,0 & 30,4 & 6,4 & 0,994 & $-13,0$ & 300,0 & 182,8 & 15,389 \\
\hline 17 & 0,0 & 31,9 & 6,7 & 0,990 & $-13,3$ & 320,0 & 175,7 & 15,284 \\
\hline 18 & 0,0 & 33,4 & 7,0 & 0,986 & $-13,6$ & 340,0 & 168,4 & 9,628 \\
\hline 19 & 0,0 & 35,0 & 7,4 & 0,981 & $-14,0$ & 360,0 & 160,7 & 9,452 \\
\hline 20 & 0,0 & 36,5 & 7,7 & 0,975 & $-14,3$ & 380,0 & 152,7 & 9,273 \\
\hline 21 & 0,0 & 38,0 & 8,0 & 0,970 & $-14,7$ & 400,0 & 144,5 & 9,080 \\
\hline 22 & 0,0 & 39,5 & 8,3 & 0,963 & $-15,0$ & 420,0 & 136,0 & 8,871 \\
\hline 23 & 0,0 & 41,0 & 8,6 & 0,957 & $-15,4$ & 440,0 & 127,1 & 8,649 \\
\hline 24 & 0,0 & 42,6 & 9,0 & 0,950 & $-15,8$ & 460,0 & 118,0 & 8,397 \\
\hline 25 & 0,0 & 44,1 & 9,3 & 0,943 & $-16,2$ & 480,0 & 108,6 & 8,136 \\
\hline 26 & 0,0 & 45,6 & 9,6 & 0,935 & $-16,6$ & 500,0 & 98,9 & 7,846 \\
\hline 27 & 0,0 & 47,1 & 9,9 & 0,926 & $-17,1$ & 520,0 & 88,9 & 7,526 \\
\hline 28 & 0,0 & 48,6 & 10,2 & 0,916 & $-17,6$ & 540,0 & 78,6 & 7,175 \\
\hline 29 & 0,0 & 50,2 & 10,6 & 0,906 & $-18,1$ & 560,0 & 67,9 & 6,762 \\
\hline 30 & 0,0 & 51,7 & 10,9 & 0,894 & $-18,7$ & 580,0 & 57,0 & 6,307 \\
\hline 31 & 0,0 & 53,2 & 11,2 & 0,881 & $-19,3$ & 600,0 & 45,7 & 5,769 \\
\hline 32 & 0,0 & 54,7 & 11,5 & 0,866 & $-20,0$ & 620,0 & 34,1 & 5,114 \\
\hline 33 & 0,0 & 56,2 & 11,8 & 0,846 & $-20,9$ & 640,0 & 22,2 & 4,279 \\
\hline 34 & 0,0 & 57,8 & 12,2 & 0,819 & $-22,1$ & 660,0 & 10,0 & 2,988 \\
\hline 35 & 0,0 & 58,5 & 12,3 & 0,798 & $-23,0$ & 670,0 & 3,8 & 1,919 \\
\hline 36 & 0,0 & 58,9 & 12,4 & 0,769 & $-24,2$ & 675,0 & 0,1 & 0,225 \\
\hline 37 & 0,0 & 58,9 & 12,4 & 0,765 & $-24,3$ & 675,0 & 0,0 & 0,225 \\
\hline 38 & 0,0 & 58,9 & 12,4 & 0,764 & $-24,4$ & 675,0 & 0,0 & 0,225 \\
\hline 39 & 0,0 & 58,9 & 12,4 & 0,760 & $-24,5$ & 675,0 & 0,0 & $-0,229$ \\
\hline 40 & 0,0 & 58,9 & 12,4 & 0,756 & $-24,7$ & 675,0 & $-0,1$ & $-0,229$ \\
\hline 41 & 0,0 & 58,9 & 12,4 & 0,752 & $-24,8$ & 675,0 & $-0,3$ & $-0,473$ \\
\hline & & & & & & & & \\
\hline
\end{tabular}

O índice $M_{Q V}$ diminui com o aumento da carga, indicando a aproximação ao lado esquerdo da curva QV. A partir do Caso 40 os índices $M_{Q V}$ e $\left(\frac{\partial Q}{\partial V}\right)$ " passam a apresentar valores negativos, indicando operação fora da região normal.

Após o Caso 40 o índice $M_{Q V}$ continua diminuindo com o aumento da carga demandada pelo Sistema nas barras 4 e 5 . A análise da barra 5 não sinaliza um retorno a zero do índice $M_{Q V}$, portanto, não apresentando a inversão da sensibilidade tensão/potência nessa barra. 
A simulação de FPC diminui a carga até o Caso 86, quando o aumento de carga volta a ser positivo, ocorrendo o aumento da $M_{Q V}$. No Caso 111 ocorre uma nova carga máxima.

Na barra 5 também é possível perceber a perda de continuidade do índice $\left(\frac{\partial Q}{\partial V}\right)$ "nos Casos 14, 15 e 18 quando ocorre perda de tensão controlada das barras do tipo PV.

Assim como ocorreu para a barra 4, os Casos 36 a 41 representam a carga máxima e até o Caso 35 ambos os índices são positivos como esperado. No Caso 37 o índice $M_{Q V}$ fica nulo e entre os Casos 38 e 39 o índice $\left(\frac{\partial Q}{\partial V}\right)$ " troca de sinal ficando negativo. A partir daí o interesse prático é pequeno.

Na Figura 5.9 observa-se o panorama geral do comportamento do índice $\left(\frac{\partial Q}{\partial V}\right)$ "versus o aumento de carga nas barras 4 e 5 . Pode-se notar a deterioração do índice $\left(\frac{\partial Q}{\partial V}\right)$ " com o aumento dos patamares de carga e as descontinuidades que ocorrem quando da perda de geração nas barras do tipo PV.

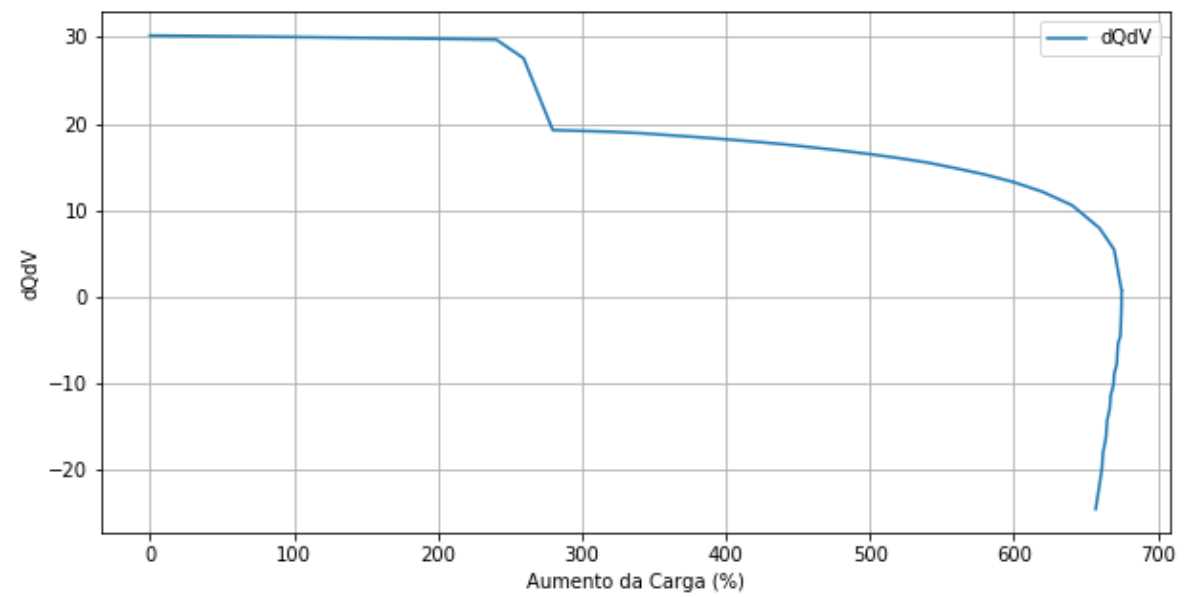

Figura 5.9: índice $\left(\frac{\partial Q}{\partial V}\right) "$ na Barra 5

\subsubsection{5}

\section{Conclusão}

Para a barra 1 existe uma região de operação em que o índice de margem da curva QV é nulo, mas o índice $\left(\frac{\partial Q}{\partial V}\right)$ " não. Essa divergência de resultados nos índices $M_{Q V}$ e $\left(\frac{\partial Q}{\partial V}\right)$ " ocorre entre os Casos 17 ao 21 para a barra 1 . A máxima carga só ocorre nos Casos 36 a 41.

Essa divergência nos resultados na barra 1 demostra que em regiões de máximo carregamento a avaliação de estabilidade de tensão pode não ser conclusiva. 
Nas demais barras analisadas (2, 4 e 5) não foi detectada essa divergência de resultados entre os índices.

Também é interessante notar que o FPC apresenta uma primeira carga máxima entre os Casos 36 a 41, após esse caso o carregamento nas barras 4 e 5 diminui até o Caso 86, quando a carga volta a aumentar até o segundo carregamento máximo no Caso 111.

As barras 2, 4 e 5 tem os índices $M_{Q V}$ e $\left(\frac{\partial Q}{\partial V}\right) "$ indicando que o sistema não opera na região usual a partir do Caso 41.

Porém, para a barra 1 os índices indicam operação fora da região usual desde o Caso 17, quando passam a apresentar resultados negativos.

\section{3}

\section{Incongruência na Margem de Potência Reativa da Curva QV Obtida Através do Fluxo de Potência Continuado}

O algoritmo do fluxo de potência continuado (FPC) inicia-se em um caso-base, e posteriormente aumenta-se o patamar de carga do sistema, obtendo-se um novo caso para execução do fluxo de potência. O procedimento é repetido diversas vezes, de tal forma que a simulação do FPC caminha para um ponto crítico denominado ponto de máximo carregamento [17].

O aumento de carga é definido por um determinado percentual de incremento e pode ser feito em uma ou mais barras. Para realização do FPC é utilizado o software ANAREDE [14].

E para todas tabelas dessa seção a Margem da Curva QV determina a distância em MW do ponto de máximo carregamento.

\subsection{1}

\section{Comparação das Cargas Máximas da Curva QV}

O sistema utilizado para simulação é o IEEE 14 barras definido na Seção 5.2. O FPC é configurado com um percentual de aumento de carga nas barras 4 e 5 , e a barra 2 possui o papel de compensar o aumento de carga com sua geração, enquando a barra 1 absorve as perdas de potência ativa.

Aqui o objetivo é avaliar a consistência e a robustez dos estudos de estabilidade de tensão utilizando a ferramenta do FPC. Para isso, na Tabela 5.11, apresenta-se uma comparação do ponto de carregamento máximo obtido para seis simulações do FPC com diferentes percentuais de aumento da carga do sistema. 
Tabela 5.11: Comparação das Cargas Máximas da Curva QV

\begin{tabular}{|c|c|c|c|c|c|c|}
\hline \multicolumn{7}{|c|}{ Carga Máxima } \\
\hline Aumento de Carga (\%) & 5,0 & 6,5 & 10,0 & 15,0 & 20,0 & 25,0 \\
\hline Ativa (MW) & 360,83 & 360,82 & 462,22 & 462,68 & 462,43 & 462,17 \\
\hline Reativa (Mvar) & $-14,98$ & $-14,98$ & $-19,19$ & $-19,21$ & $-19,2$ & $-19,19$ \\
\hline
\end{tabular}

O resultado da Tabela 5.11 indica valores menores para o máximo carregamento do sistema quando o percentual de incremento da simulação de FPC é menor que $10 \%$.

Nas Tabelas 5.12 e 5.13 são detalhados alguns casos de interesse da simulação de FPC para os percentuais de aumento de carga de 5\% e 10\%, respectivamente. Cada linha das tabelas corresponde a um caso com seu percentual de aumento de carga, e as cargas ativa e reativa correspondentes.

Nas Tabelas 5.12 e 5.13 é observado como a carga ativa e reativa aumentam em termos de valores absolutos e após atingir o caso de maior carregamento, o incremento de carga diminui até cessar. Na Tabela 5.12 a carga ativa máxima é de 360,83 MW (Caso 123). E na Tabela 5.13 a carga ativa máxima é de 462,22 MW (Caso 92).

Esse resultado demonstra que simulações com diferentes taxas percentuais de aumentos de carga levam a valores diferentes de carga máxima, o que vem a ser uma incongruência nesse estudo.

\subsection{2}

\section{Comparação da Margem da Curva QV}

Conforme definido na Seção 4.3, a margem da curva QV é um resultado importante da curva QV e avalia o nível de carregamento desse sistema.

Nas Tabelas 5.14, 5.15, 5.16, 5.17 e 5.18 são apresentadas três simulações com o percentual de aumento de carga de 5\%,10\% e 20\% em cinco cenários (patamares de carga) diferentes. O estudo comparativo da margem da curva QV é composto pelas seguintes variáveis do caso simulado: carga ativa, o lado da curva QV, a margem da curva QV. Além disso, é informado a potência reativa injetada ( $\mathrm{Q}$ injetada) no caso-base e a potência reativa no ponto de fronteira da curva QV (Q inflexão) para o caso simulado. Vale ressaltar que nas três simulações a carga ativa é idêntica.

A barra 1 é a escolhida para obter a curva QV, barra swing.

As cinco tabelas apresentadas são compostas pelo mesmo conjunto de informações, mas em cada tabela a carga ativa possui nível de carga diferente. 
É verificado que a margem da curva QV na simulação com percentual de aumento de carga de $5 \%$ é diferente do apresentado nas simulações com percentual de aumento de carga de $10 \%$ e $20 \%$. Isso ocorre mesmo com a carga ativa idêntica para todos os casos. Ou seja, para um mesmo sistema e mesma carga, a curva QV fornece diferentes valores para a margem da curva QV dependendo do percentual de incremento de carga empregado.

Por último, nota-se que a potência reativa injetada e a potência reativa no ponto de fronteira são diferentes para a simulação com aumento de carga de $5 \%$ e para as simulações com aumento de carga de $10 \%$ e $20 \%$.

Essa diferença ocorre porque com $5 \%$ são necessários quatro aumentos de carga, enquanto com $10 \%$ são dois e com $20 \%$ um aumento é suficiente. Embora a carga ativa e reativa seja a mesma nos três casos, a potência reativa gerada pela barra 1 vai ficando diferente à medida que diferentes aumentos de carga são feitos e mais resoluções do problema de fluxo de carga são necessárias. 
Tabela 5.12: Casos do FPC com percentual de aumento de carga de 5\%

\begin{tabular}{|c|c|c|c|}
\hline Caso & $\begin{array}{c}\text { Aumento } \\
\text { de Carga (\%) }\end{array}$ & $\begin{array}{c}\text { Carga Reativa } \\
\text { (Mvar) }\end{array}$ & $\begin{array}{c}\text { Carga Ativa } \\
(\mathrm{MW})\end{array}$ \\
\hline 0 & 0,0 & $-2,3$ & 55,4 \\
\hline 1 & 5,0 & $-2,42$ & 58,17 \\
\hline 2 & 10,0 & $-2,53$ & 60,94 \\
\hline 3 & 15,0 & $-2,65$ & 63,71 \\
\hline 4 & 20,0 & $-2,76$ & 66,48 \\
\hline 5 & 25,0 & $-2,88$ & 69,25 \\
\hline 6 & 30,0 & $-2,99$ & 72,02 \\
\hline 7 & 35,0 & $-3,11$ & 74,79 \\
\hline 8 & 40,0 & $-3,22$ & 77,56 \\
\hline 9 & 45,0 & $-3,34$ & 80,33 \\
\hline 10 & 50,0 & $-3,45$ & 83,1 \\
\hline 11 & 55,0 & $-3,57$ & 85,87 \\
\hline 12 & 60,0 & $-3,68$ & 88,64 \\
\hline 13 & 65,0 & $-3,8$ & 91,41 \\
\hline 14 & 70,0 & $-3,91$ & 94,18 \\
\hline 105 & 525,0 & $-14,38$ & 346,25 \\
\hline 106 & 530,0 & $-14,49$ & 349,02 \\
\hline 107 & 535,0 & $-14,61$ & 351,79 \\
\hline 108 & 540,0 & $-14,72$ & 354,56 \\
\hline 109 & 545,0 & $-14,84$ & 357,33 \\
\hline 110 & 550,0 & $-14,95$ & 360,1 \\
\hline 113 & 551,25 & $-14,98$ & 360,79 \\
\hline 118 & 551,25 & $-14,98$ & 360,79 \\
\hline 122 & 551,29 & $-14,98$ & 360,82 \\
\hline 123 & 551,31 & $-14,98$ & 360,83 \\
\hline 124 & 551,22 & $-14,98$ & 360,78 \\
\hline 125 & 551,03 & $-14,97$ & 360,67 \\
\hline 126 & 550,74 & $-14,97$ & 360,51 \\
\hline 127 & 550,35 & $-14,96$ & 360,3 \\
\hline 128 & 549,86 & $-14,95$ & 360,03 \\
\hline 129 & 549,28 & $-14,93$ & 359,7 \\
\hline 130 & 548,60 & $-14,92$ & 359,33 \\
\hline 131 & 547,83 & $-14,9$ & 358,9 \\
\hline 132 & 546,97 & $-14,88$ & 358,43 \\
\hline 133 & 546,02 & $-14,86$ & 357,9 \\
\hline 134 & 544,99 & $-14,83$ & 357,33 \\
\hline 135 & 543,87 & $-14,81$ & 356,71 \\
\hline 136 & 542,67 & $-14,78$ & 356,04 \\
\hline 137 & 541,38 & $-14,75$ & 355,33 \\
\hline 138 & 540,02 & $-14,72$ & 354,57 \\
\hline
\end{tabular}


Tabela 5.13: Casos do FPC com percentual de aumento de carga de $10 \%$

\begin{tabular}{|c|c|c|c|}
\hline Caso & $\begin{array}{c}\text { Aumento } \\
\text { de Carga (\%) }\end{array}$ & $\begin{array}{c}\text { Carga Reativa } \\
\text { (Mvar) }\end{array}$ & $\begin{array}{c}\text { Carga Ativa } \\
\text { (MW) }\end{array}$ \\
\hline 0 & 0 & $-2,3$ & 55,4 \\
\hline 1 & 10 & $-2,53$ & 60,94 \\
\hline 2 & 20 & $-2,76$ & 66,48 \\
\hline 3 & 30 & $-2,99$ & 72,02 \\
\hline 4 & 40 & $-3,22$ & 77,56 \\
\hline 5 & 50 & $-3,45$ & 83,1 \\
\hline 6 & 60 & $-3,68$ & 88,64 \\
\hline 7 & 70 & $-3,91$ & 94,18 \\
\hline 8 & 80 & $-4,14$ & 99,72 \\
\hline 9 & 90 & $-4,37$ & 105,26 \\
\hline 10 & 100 & $-4,6$ & 110,8 \\
\hline 11 & 110 & $-4,83$ & 116,34 \\
\hline 12 & 120 & $-5,06$ & 121,88 \\
\hline 13 & 130 & $-5,29$ & 127,42 \\
\hline 14 & 140 & $-5,52$ & 132,96 \\
\hline 65 & 650 & $-17,25$ & 415,5 \\
\hline 66 & 660 & $-17,48$ & 421,04 \\
\hline 67 & 670 & $-17,71$ & 426,58 \\
\hline 68 & 680 & $-17,94$ & 432,12 \\
\hline 69 & 690 & $-18,17$ & 437,66 \\
\hline 70 & 700 & $-18,4$ & 443,2 \\
\hline 71 & 710 & $-18,63$ & 448,74 \\
\hline 72 & 720 & $-18,86$ & 454,28 \\
\hline 73 & 730 & $-19,09$ & 459,82 \\
\hline 76 & 732,5 & $-19,15$ & 461,21 \\
\hline 78 & 733,75 & $-19,18$ & 461,9 \\
\hline 81 & 734,063 & $-19,18$ & 462,07 \\
\hline 83 & 734,219 & $-19,19$ & 462,16 \\
\hline 85 & 734,297 & $-19,19$ & 462,2 \\
\hline 86 & 734,297 & $-19,19$ & 462,2 \\
\hline 91 & 734,323 & $-19,19$ & 462,21 \\
\hline 92 & 734,325 & $-19,19$ & 462,22 \\
\hline 93 & 734,201 & $-19,19$ & 462,15 \\
\hline 94 & 733,952 & $-19,18$ & 462,01 \\
\hline 95 & 733,582 & $-19,17$ & 461,8 \\
\hline 96 & 733,091 & $-19,16$ & 461,53 \\
\hline 97 & 732,483 & $-19,15$ & 461,2 \\
\hline 98 & 731,759 & $-19,13$ & 460,79 \\
\hline 99 & 730,922 & $-19,11$ & 460,33 \\
\hline 100 & 729,973 & $-19,09$ & 459,8 \\
\hline
\end{tabular}


Tabela 5.14: Cenário 1 das simulação de FPC com aumento de carga de 5\%, $10 \%$ e $20 \%$

\begin{tabular}{|c|c|c|c|c|c|c|}
\hline \multicolumn{7}{|c|}{ Comparando curva QV } \\
\hline $\begin{array}{c}\text { Aumento } \\
\text { de Carga } \\
(\mathbf{\%})\end{array}$ & Caso & $\begin{array}{c}\text { Carga } \\
\text { Ativa } \\
(\mathbf{M W})\end{array}$ & $\begin{array}{c}\text { Lado } \\
\text { Curva QV }\end{array}$ & $M_{Q V}$ & $\begin{array}{c}\text { Qinjetada } \\
(\text { Mvar) }\end{array}$ & $\begin{array}{c}\text { Qinflexão } \\
\text { (Mvar) }\end{array}$ \\
\hline 5 & 4 & 66,48 & DIR & $-26,6$ & $-18,1$ & $-44,7$ \\
\hline 10 & 2 & 66,48 & DIR & $-35,1$ & $-13,3$ & $-48,36$ \\
\hline 20 & 1 & 66,48 & DIR & $-35,1$ & $-13,3$ & $-48,36$ \\
\hline
\end{tabular}

Tabela 5.15: Cenário 2 das simulação de FPC com aumento de carga de 5\%, $10 \%$ e $20 \%$

\begin{tabular}{|c|c|c|c|c|c|c|}
\hline \multicolumn{7}{|c|}{ Comparando curva QV } \\
\hline $\begin{array}{c}\text { Aumento } \\
\text { de Carga } \\
(\%)\end{array}$ & Caso & $\begin{array}{c}\text { Carga } \\
\text { Ativa } \\
(\mathbf{M W})\end{array}$ & $\begin{array}{c}\text { Lado } \\
\text { Curva QV }\end{array}$ & $M_{Q V}$ & $\begin{array}{c}\text { Qinjetada } \\
\text { (Mvar) }\end{array}$ & $\begin{array}{c}\text { Qinflexão } \\
\text { (Mvar) }\end{array}$ \\
\hline 5 & 8 & 77,56 & DIR & $-20,9$ & $-19,3$ & $-40,23$ \\
\hline 10 & 4 & 77,56 & DIR & $-38,5$ & -10 & $-48,45$ \\
\hline 20 & 2 & 77,56 & DIR & $-38,5$ & -10 & $-48,45$ \\
\hline
\end{tabular}

Tabela 5.16: Cenário 3 das simulação de FPC com aumento de carga de 5\%, $10 \%$ e $20 \%$

\begin{tabular}{|c|c|c|c|c|c|c|}
\hline \multicolumn{7}{|c|}{ Comparando curva QV } \\
\hline $\begin{array}{c}\text { Aumento } \\
\text { de Carga } \\
(\mathbf{\%})\end{array}$ & Caso & $\begin{array}{c}\text { Carga } \\
\text { Ativa } \\
(\mathbf{M W})\end{array}$ & $\begin{array}{c}\text { Lado } \\
\text { Curva QV }\end{array}$ & $M_{Q V}$ & $\begin{array}{c}\text { Qinjetada } \\
\text { (Mvar) }\end{array}$ & $\begin{array}{c}\text { Qinflexão } \\
\text { (Mvar) }\end{array}$ \\
\hline 5 & 16 & 99,72 & DIR & $-14,4$ & $-17,3$ & $-31,7$ \\
\hline 10 & 8 & 99,72 & DIR & $-44,5$ & $-3,1$ & $-47,57$ \\
\hline 20 & 4 & 99,72 & DIR & $-44,5$ & $-3,1$ & $-47,57$ \\
\hline
\end{tabular}

Tabela 5.17: Cenário 4 das simulação de FPC com aumento de carga de 5\%, $10 \%$ e $20 \%$

\begin{tabular}{|c|c|c|c|c|c|c|}
\hline \multicolumn{7}{|c|}{ Comparando curva QV } \\
\hline $\begin{array}{c}\text { Aumento } \\
\text { de Carga } \\
(\mathbf{\%})\end{array}$ & Caso & $\begin{array}{c}\text { Carga } \\
\text { Ativa } \\
(\mathbf{M W})\end{array}$ & $\begin{array}{c}\text { Lado } \\
\text { Curva QV }\end{array}$ & $M_{Q V}$ & $\begin{array}{c}\text { Qinjetada } \\
(\text { Mvar) }\end{array}$ & $\begin{array}{c}\text { Qinflexão } \\
\text { (Mvar) }\end{array}$ \\
\hline 5 & 32 & 144,04 & DIR & $-1,7$ & $-11,1$ & $-12,71$ \\
\hline 10 & 16 & 144,04 & DIR & $-52,6$ & 11,4 & $-41,13$ \\
\hline 20 & 8 & 144,04 & DIR & $-52,6$ & 11,4 & $-41,13$ \\
\hline
\end{tabular}


Tabela 5.18: Cenário 5 das simulação de FPC com aumento de carga de 5\%, $10 \%$ e $20 \%$

\begin{tabular}{|c|c|c|c|c|c|c|}
\hline \multicolumn{7}{|c|}{ Comparando curva QV } \\
\hline $\begin{array}{c}\text { Aumento } \\
\text { de Carga } \\
(\mathbf{\%})\end{array}$ & Caso & $\begin{array}{c}\text { Carga } \\
\text { Ativa } \\
(\mathbf{M W})\end{array}$ & $\begin{array}{c}\text { Lado } \\
\text { Curva QV }\end{array}$ & $M_{Q V}$ & $\begin{array}{c}\text { Qinjetada } \\
\text { (Mvar) }\end{array}$ & $\begin{array}{c}\text { Qinflexão } \\
\text { (Mvar) }\end{array}$ \\
\hline 5 & 64 & 232,68 & ESQ & $-16,9$ & 54,2 & 37,29 \\
\hline 10 & 32 & 232,68 & DIR & $-55,3$ & 45,4 & $-9,91$ \\
\hline 20 & 16 & 232,68 & DIR & $-55,3$ & 45,4 & $-9,91$ \\
\hline
\end{tabular}

\subsection{3}

\section{Margem da Curva QV para Diferentes Mecanismos de Aumento de Carga}

$\mathrm{O}$ incremento de carga realizado no método de FPC pode ser realizado aplicando uma progressão aritmética ou geométrica à carga inicial do sistema estudado. A maneira que é escolhida para aumentar o patamar tem forte influência na rapidez com que o FPC atinge seu carregamento máximo.

Primeiramente, considera-se que o aumento de carga do FPC é realizado como uma progressão aritmética (PA), com um aumento de 10\%. Se uma carga inicial de $10 \mathrm{MW}$ é considerada para o caso-base (caso 0), tem-se para o caso 1 uma carga de 11 MW. Já para o caso 2, uma carga de 12 MW. E para o caso 3, uma carga de $13 \mathrm{MW}$ e assim sucessivamente.

Por outro lado, considere-se o caso em que o aumento de carga do FPC é realizado como uma progressão geométrica (PG), com um aumento de $10 \%$. Se uma carga inicial de $10 \mathrm{MW}$ é considerada para o caso-base (caso 0), tem-se para o caso 1 uma carga de 11 MW. Para o caso 2, uma carga de 12,1 MW. E para o caso 3, uma carga de 13,31 MW e assim sucessivamente.

Em [15] é utilizado a versão 10.0.1 do ANAREDE, que implementa o aumento de carga do FPC com PG.

Com o objetivo de melhor compreender os resultados incongruentes para o FPC apresentados na SubSeção 5.3.2, comparam-se as margens de curva QV do FPC da versão 10.0.1 do ANAREDE com o FPC da versão 10.2.3 do ANAREDE. Na versão 10.2.3 o aumento de carga é feito através de PA.

Aqui novamente, assim como nos resultados da Seção 5.3.2, pretende-se analisar casos do FPC com a mesma carga ativa. E entre esses casos compara-se suas margens da curva QV, potências reativas injetadas e potências no ponto de fronteira da curva QV. Porém, nesse estudo tem-se um procedimento diferente para realizar o incremento de carga. Portanto, serão analisados dois casos com patamares de carga semelhantes, já que patamares idênticos é impossível. 
Primeiramente foi estudado o FPC com percentual de aumento de carga de $5 \%$ para as duas versões do ANAREDE e os resultados podem ser vistos nas Tabelas 5.19, 5.20 e 5.21 .

No Cenário 1, a margem da curva QV é aproximadamente a mesma para ambos os casos apresentados. No Cenário 2, com carga ativa maior, observa-se o mesmo comportamento. Já no Cenário 3, o que possui maior nível de carga dentre todos, os casos apresentam margem da curva QV diferente mesmo com níveis de carga ativa semelhante. Deve-se ressaltar que, quanto maior a carga, maior será o número de resoluções do fluxo de potência são executadas. Portanto, com o aumento de carga em PA é necessário executar maior número de resoluções de fluxo de potência do que com o aumento de carga em PG. Assim, as potências reativas geradas na barra 1 tornam-se, gradualmente, diferentes.

Tabela 5.19: Cenário 1 das versões do ANAREDE para aumento de carga do $\mathrm{FPC}$ com $5 \%$

\begin{tabular}{|c|c|c|c|c|c|c|}
\hline \multicolumn{7}{|c|}{ Comparando versões ANAREDE - Cenário 1 } \\
\hline versão & Caso & $\begin{array}{c}\text { Carga } \\
\text { ativa (MW) }\end{array}$ & $\begin{array}{c}\text { Lado } \\
\text { Curva QV }\end{array}$ & $M_{Q V}$ & $\begin{array}{c}\text { Q injetada } \\
\text { (Mvar) }\end{array}$ & $\begin{array}{c}\text { Q inflexão } \\
\text { (Mvar) }\end{array}$ \\
\hline 10.0 .1 & 6 & 74,24 & DIR & $-22,1$ & $-19,2$ & $-41,3$ \\
\hline 10.2 .3 & 7 & 74,29 & DIR & $-21,8$ & $-19,3$ & $-41,06$ \\
\hline
\end{tabular}

Tabela 5.20: Cenário 2 das versões do ANAREDE para aumento de carga do FPC com $5 \%$

\begin{tabular}{|c|c|c|c|c|c|c|}
\hline \multicolumn{7}{|c|}{ Comparando versões ANAREDE - Cenário 2 } \\
\hline versão & Caso & $\begin{array}{c}\text { Carga } \\
\text { ativa (MW) }\end{array}$ & $\begin{array}{c}\text { Lado } \\
\text { Curva QV }\end{array}$ & $M_{Q V}$ & $\begin{array}{c}\text { Q injetada } \\
\text { (Mvar) }\end{array}$ & $\begin{array}{c}\text { Q inflexão } \\
\text { (Mvar) }\end{array}$ \\
\hline 10.0 .1 & 9 & 85,94 & DIR & $-19,2$ & $-18,6$ & $-37,81$ \\
\hline 10.2 .3 & 11 & 85,87 & DIR & $-19,2$ & $-18,6$ & $-37,83$ \\
\hline
\end{tabular}

Tabela 5.21: Cenário 3 das versões do ANAREDE para aumento de carga do FPC com $5 \%$

\begin{tabular}{|c|c|c|c|c|c|c|}
\hline \multicolumn{7}{|c|}{ Comparando versões ANAREDE - Cenário 3 } \\
\hline versão & Caso & $\begin{array}{c}\text { Carga } \\
\text { ativa (MW) }\end{array}$ & $\begin{array}{c}\text { Lado } \\
\text { Curva QV }\end{array}$ & $M_{Q V}$ & $\begin{array}{c}\text { Q injetada } \\
\text { (Mvar) }\end{array}$ & $\begin{array}{c}\text { Q inflexão } \\
\text { (Mvar) }\end{array}$ \\
\hline 10.0 .1 & 26 & 196,98 & DIR & 0 & $-7,3$ & $-7,3$ \\
\hline 10.2 .3 & 51 & 196,67 & ESQ & $-5,4$ & $-21,2$ & 15,74 \\
\hline
\end{tabular}

No segundo estudo com o FPC, o percentual de aumento de carga é de $6,5 \%$ para as duas versões do ANAREDE. Os resultados podem ser vistos nas Tabelas 5.22, 5.23, 5.24 e 5.25 . 
No Cenário 1, a margem da curva QV é aproximadamente a mesma para ambos os casos apresentados. No Cenário 2, com carga ativa maior, observa-se o mesmo comportamento. Já nos Cenários 3 e 4, o que possuem maior nível de carga, os casos apresentam margem da curva QV diferentes mesmo com níveis de carga ativa semelhante.

Nesse segundo estudo os Cenários comparativos apresentam potência reativas diferentes na barra 1 para os Cenários de maior carregamento (Cenários 3 e 4)

Tabela 5.22: Cenário 1 das versões do ANAREDE para aumento de carga do FPC com $6.5 \%$

\begin{tabular}{|c|c|c|c|c|c|c|}
\hline \multicolumn{7}{|c|}{ Comparando versões ANAREDE - Cenário 1 } \\
\hline versão & Caso & $\begin{array}{c}\text { Carga } \\
\text { ativa (MW) }\end{array}$ & $\begin{array}{c}\text { Lado } \\
\text { Curva QV }\end{array}$ & $M_{Q V}$ & $\begin{array}{c}\text { Q injetada } \\
\text { (Mvar) }\end{array}$ & $\begin{array}{c}\text { Q inflexão } \\
\text { (Mvar) }\end{array}$ \\
\hline 10.0 .1 & caso 7 & 80,61 & DIR & $-20,5$ & $-19,1$ & $-39,58$ \\
\hline 10.2 .3 & caso 6 & 80,64 & DIR & $-20,5$ & $-19,1$ & $-39,58$ \\
\hline
\end{tabular}

Tabela 5.23: Cenário 2 das versões do ANARinEDE para aumento de carga do FPC com $6.5 \%$

\begin{tabular}{|c|c|c|c|c|c|c|}
\hline \multicolumn{7}{|c|}{ Comparando versões ANAREDE - Cenário 2 } \\
\hline versão & Caso & $\begin{array}{c}\text { Carga } \\
\text { ativa (MW) }\end{array}$ & $\begin{array}{c}\text { Lado } \\
\text { Curva QV }\end{array}$ & $M_{Q V}$ & $\begin{array}{c}\text { Q injetada } \\
\text { (Mvar) }\end{array}$ & $\begin{array}{c}\text { Q inflexão } \\
\text { (Mvar) }\end{array}$ \\
\hline 10.0 .1 & caso 10 & 91,41 & DIR & $-17,7$ & $-18,1$ & $-35,84$ \\
\hline 10.2 .3 & caso 8 & 91,69 & DIR & $-19,2$ & $-18,7$ & $-37,89$ \\
\hline
\end{tabular}

Tabela 5.24: Cenário 3 das versões do ANAREDE para aumento de carga do FPC com $6.5 \%$

\begin{tabular}{|c|c|c|c|c|c|c|}
\hline \multicolumn{7}{|c|}{ Comparando versões ANAREDE - Cenário 3 } \\
\hline versão & Caso & $\begin{array}{c}\text { Carga } \\
\text { ativa (MW) }\end{array}$ & $\begin{array}{c}\text { Lado } \\
\text { Curva QV }\end{array}$ & $M_{Q V}$ & $\begin{array}{c}\text { Q injetada } \\
\text { (Mvar) }\end{array}$ & $\begin{array}{c}\text { Q inflexão } \\
\text { (Mvar) }\end{array}$ \\
\hline 10.0 .1 & caso 39 & 195,84 & ESQ & $-4,9$ & 20,5 & 15,54 \\
\hline 10.2 .3 & caso 20 & 195,21 & DIR & 0 & $-10,5$ & $-10,46$ \\
\hline
\end{tabular}

Tabela 5.25: Cenário 4 das versões do ANAREDE para aumento de carga do FPC com $6.5 \%$

\begin{tabular}{|c|c|c|c|c|c|c|}
\hline \multicolumn{7}{|c|}{ Comparando versões ANAREDE - Cenário 4 } \\
\hline versão & Caso & $\begin{array}{c}\text { Carga } \\
\text { ativa (MW) }\end{array}$ & $\begin{array}{c}\text { Lado } \\
\text { Curva QV }\end{array}$ & $M_{Q V}$ & $\begin{array}{c}\text { Q injetada } \\
\text { (Mvar) }\end{array}$ & $\begin{array}{c}\text { Q inflexão } \\
\text { (Mvar) }\end{array}$ \\
\hline 10.0 .1 & caso 34 & 221,05 & ESQ & $-10,6$ & 42,7 & 32,12 \\
\hline 10.2 .3 & caso 20 & 221,41 & DIR & 0 & $-1,8$ & $-1,8$ \\
\hline
\end{tabular}


Um terceiro estudo com o FPC com percentual de aumento de carga de $10 \%$ para as duas versões do ANAREDE e os resultados podem ser vistos nas Tabelas 5.26, 5.27, 5.28.

Nos Cenários 1, 2 e 3, as margens da curva QV são bem diferentes para as duas versões do programa.

Tabela 5.26: Cenário 1 das versões do ANAREDE para aumento de carga do FPC com $10 \%$

\begin{tabular}{|c|c|c|c|c|c|c|}
\hline \multicolumn{7}{|c|}{ Comparando versões ANAREDE - Cenário 1 } \\
\hline versão & Caso & $\begin{array}{c}\text { Carga } \\
\text { ativa (MW) }\end{array}$ & $\begin{array}{c}\text { Lado } \\
\text { Curva QV }\end{array}$ & $M_{Q V}$ & $\begin{array}{c}\text { Q injetada } \\
\text { (Mvar) }\end{array}$ & $\begin{array}{c}\text { Q inflexão } \\
\text { (Mvar) }\end{array}$ \\
\hline 10.0 .1 & 14 & 210,38 & DIR & $-5,5$ & $-3,2$ & $-8,68$ \\
\hline 10.2 .3 & 28 & 210,52 & DIR & $-56,4$ & 35,8 & $-20,63$ \\
\hline
\end{tabular}

Tabela 5.27: Cenário 2 das versões do ANAREDE para aumento de carga do FPC com $10 \%$

\begin{tabular}{|c|c|c|c|c|c|c|}
\hline \multicolumn{7}{|c|}{ Comparando versões ANAREDE - Cenário 2 } \\
\hline versão & Caso & $\begin{array}{c}\text { Carga } \\
\text { ativa (MW) }\end{array}$ & $\begin{array}{c}\text { Lado } \\
\text { Curva QV }\end{array}$ & $M_{Q V}$ & $\begin{array}{c}\text { Q injetada } \\
\text { (Mvar) }\end{array}$ & $\begin{array}{c}\text { Q inflexão } \\
\text { (Mvar) }\end{array}$ \\
\hline 10.0 .1 & 16 & 254,56 & DIR & 0 & 10,4 & 10,4 \\
\hline 10.2 .3 & 36 & 254,84 & DIR & $-52,6$ & 55,4 & 2,75 \\
\hline
\end{tabular}

Tabela 5.28: Cenário 3 das versões do ANAREDE para aumento de carga do FPC com $10 \%$

\begin{tabular}{|c|c|c|c|c|c|c|}
\hline \multicolumn{7}{|c|}{ Comparando versões ANAREDE - Cenário 3 } \\
\hline versão & Caso & $\begin{array}{c}\text { Carga } \\
\text { ativa (MW) }\end{array}$ & $\begin{array}{c}\text { Lado } \\
\text { Curva QV }\end{array}$ & $M_{Q V}$ & $\begin{array}{c}\text { Q injetada } \\
\text { (Mvar) }\end{array}$ & $\begin{array}{c}\text { Q inflexão } \\
\text { (Mvar) }\end{array}$ \\
\hline 10.0 .1 & 21 & 409,97 & ESQ & $-68,6$ & 189,7 & 121,14 \\
\hline 10.2 .3 & 64 & 409,96 & ESQ & -1 & 136,2 & 135,18 \\
\hline
\end{tabular}

No quarto e último estudo com o FPC o percentual de aumento é de $15 \%$ de carga para as duas versões do ANAREDE e os resultados podem ser vistos nas Tabelas 5.29, 5.30, 5.31.

Nos Cenários 1, 2 e 3, as margens da curva QV são bem diferentes para as duas versões do programa.

Tabela 5.29: Cenário 1 das versões do ANAREDE para aumento de carga do FPC com $15 \%$

\begin{tabular}{|c|c|c|c|c|c|c|}
\hline \multicolumn{7}{|c|}{ Comparando versões ANAREDE - Cenário 1 } \\
\hline versão & Caso & $\begin{array}{c}\text { Carga } \\
\text { ativa (MW) }\end{array}$ & $\begin{array}{c}\text { Lado } \\
\text { Curva QV }\end{array}$ & $M_{Q V}$ & $\begin{array}{c}\text { Q injetada } \\
\text { (Mvar) }\end{array}$ & $\begin{array}{c}\text { Q inflexão } \\
\text { (Mvar) }\end{array}$ \\
\hline 10.0 .1 & caso 4 & 96,89 & DIR & $-27,9$ & $-13,5$ & $-41,36$ \\
\hline 10.2 .3 & caso 5 & 96,95 & DIR & $-43,9$ & -4 & $-47,88$ \\
\hline
\end{tabular}


Tabela 5.30: Cenário 2 das versões do ANAREDE para aumento de carga do FPC com $15 \%$

\begin{tabular}{|c|c|c|c|c|c|c|}
\hline \multicolumn{7}{|c|}{ Comparando versões ANAREDE - Cenário 2 } \\
\hline versão & Caso & $\begin{array}{c}\text { Carga } \\
\text { ativa (MW) }\end{array}$ & $\begin{array}{c}\text { Lado } \\
\text { Curva QV }\end{array}$ & $M_{Q V}$ & $\begin{array}{c}\text { Q injetada } \\
\text { (Mvar) }\end{array}$ & $\begin{array}{c}\text { Q inflexão } \\
\text { (Mvar) }\end{array}$ \\
\hline 10.0 .1 & caso 12 & 296,4 & ESQ & $-5,9$ & 38,3 & 32,41 \\
\hline 10.2 .3 & caso 29 & 296,39 & DIR & $-42,9$ & 74,9 & 32 \\
\hline
\end{tabular}

Tabela 5.31: Cenário 3 das versões do ANAREDE para aumento de carga do FPC com $15 \%$

\begin{tabular}{|c|c|c|c|c|c|c|}
\hline \multicolumn{7}{|c|}{ Comparando versões ANAREDE - Cenário 3 } \\
\hline versão & Caso & $\begin{array}{c}\text { Carga } \\
\text { ativa (MW) }\end{array}$ & $\begin{array}{c}\text { Lado } \\
\text { Curva QV }\end{array}$ & $M_{Q V}$ & $\begin{array}{c}\text { Q injetada } \\
\text { (Mvar) }\end{array}$ & $\begin{array}{c}\text { Q inflexão } \\
\text { (Mvar) }\end{array}$ \\
\hline 10.0 .1 & caso 15 & 421,39 & $\mathrm{ESQ}$ & $-92,6$ & 229,1 & 136,55 \\
\hline 10.2 .3 & caso 44 & 421,04 & $\mathrm{ESQ}$ & $-15,4$ & 156,1 & 140,71 \\
\hline
\end{tabular}

Assim, versões diferentes do ANAREDE podem fornecer valores diferentes para a margem da curva QV. Ou seja, dependendo do uso da PG ou PA para aumento de carga, o índice de estabilidade de tensão extraída da curva QV pode mudar. Pode-se concluir que, quanto maior é o carregamento do sistema em estudo, maior é a diferença apresentada.

\subsection{4}

\section{Conclusão}

Os resultados da presente Seção demostram que o FPC, ao efetuar o fluxo de potência diversas vezes e com alto carregamento, pode levar a resultados incoerentes nos índices de estabilidade da curva QV: lado da curva QV e a margem da curva QV.

\section{4}

\section{Sumário do Capítulo}

Os índices $\left(\frac{\partial Q}{\partial V}\right)$ " e $M_{Q V}$ indicam se o sistema opera no lado esquerdo ou direito da curva $\mathrm{QV}$, ou seja, na região usual ou não usual de operação. Porém, ao obter o $\left(\frac{\partial Q}{\partial V}\right)$ " e $M_{Q V}$ durante o estudo com aumento de carga em múltiplas barras existe uma região operativa em que a margem $M_{Q V}$ fornece valores positivos e $\left(\frac{\partial Q}{\partial V}\right)$ " fornece valores negativos.

Essa divergência nos resultados dos índices $\left(\frac{\partial Q}{\partial V}\right)$ " e $M_{Q V}$ permite concluir que existe uma região de operação, próxima a região de máximo carregamento, em que uma avaliação da estabilidade de tensão fornecer resultados incongruentes. 
A possível discrepância nos resultados dos índices $\left(\frac{\partial Q}{\partial V}\right)^{\prime \prime}$ e $M_{Q V}$ foi apresentada na Seção 4.6. Ficando evidente que os dois índices possuem formas diferentes para serem calculados, portanto, poderiam apresentar resultados diferentes.

Logo após o estudo de incongruência no resultado dos índices, na Seção 5.3 , é comparado os valores da margem $M_{Q V}$ calculada para duas versões distintas do ANAREDE. Essas versões apresentam diferentes mecanismos para realizar o aumento de carga: uma por PA e outra por PG.

Esse estudo comparativo da $M_{Q V}$ calculado para diferente versões do programa e diferentes patamares de carga, permite concluir que quando $M_{Q V}$ é obtida após efetuar-se o fluxo de potência diversas vezes no FPC, isso pode levar a valores incoerentes no lado da curva QV e na margem da curva QV. 


\section{6 \\ Conclusões}

O primeiro índice, a curva QV, é obtida executando-se o algoritmo de fluxo de potência diversas vezes. Portanto, a margem da curva QV representa uma variação de $\Delta V$ com $\Delta Q$, onde $\Delta V$ e $\Delta Q$ são variações de magnitude finita.

O segundo índice estudado, $\left(\frac{\partial Q}{\partial V}\right)^{\prime \prime}$, é determinado em um ponto de operação específico, consistindo na variação infinitesimal das grandezas Q e $\mathrm{V}$.

Conclui-se que, enquanto o índice $\left(\frac{\partial Q}{\partial V}\right)$ " avalia determinado ponto de operação, a margem da curva QV avalia um conjunto de pontos de operação.

As diferenças na maneira de se calcular e obter os dois índices são indicativos suficientes para se considerar a possível existência de divergências na interpretação dos resultados para esses dois índices.

A fim de verificar o comportamento dos índices $\left(\frac{\partial Q}{\partial V}\right)$ " e margem da curva QV foi utilizado o sistema de testes IEEE 14 barras. O programa ANAREDE simulou o sistema teste com a ferramenta de Fluxo de Potência Continuado, obtendo-se pontos de operação próximos ao ponto crítico da curva QV, e na parte inferior dessa curva.

Pontos próximos da região de inflexão da curva QV são de grande interesse, pois permitem estudar o sistema perto do seu limite de estabilidade de tensão.

No primeiro estudo de aumento de carga realizado na Seção 5.2.1 são encontradas resultados não coerentes para os índices $\left(\frac{\partial Q}{\partial V}\right)$ " e margem da curva QV. Há pontos de operação em que o índice de margem da curva QV é nulo, mas o índice $\left(\frac{\partial Q}{\partial V}\right)$ "não.

Um segundo estudo foi realizado com aumento de carga em duas barras (4 e 5), na Seção 5.2.2, sendo encontrados resultados não coerentes para os índices $\left(\frac{\partial Q}{\partial V}\right)$ " e margem da curva QV. Novamente ocorrem pontos de operação em que o índice de margem da curva $Q V$ é nulo, mas o índice $\left(\frac{\partial Q}{\partial V}\right)$ " não.

Percebeu-se que a proximidade do lado esquerdo da curva QV fornece resultados divergentes para os dois índices, esse fenômeno ocorre principalmente pela perda de controle de tensão nas demais barras do tipo 
PV, e devido a problemas numéricos para cálculo do ponto de operação na região alta não linearidade.

O método do fluxo de potência continuado utilizado para obter regiões de operação no lado esquerdo da curva QV pode fornecer diferentes resultados para os pontos de máximo carregamento, quando o fluxo de potência é executado diversas vezes.

\section{1}

\section{Trabalhos Futuros}

Este trabalho mostrou a existência de resultados incongruentes ao aplicar os índices de estabilidade de tensão $\left(\frac{\partial Q}{\partial V}\right)^{\prime}$ e margem da curva QV para avaliação da estabilidade de tensão de sistemas elétricos. Os resultados incongruentes vêm ocorrendo quando o SEE opera próximos da região de alta não linearidade e alto carregamento.

O uso do fluxo de potência continuado para obter pontos de operação em regiões do lado esquerdo da curva QV resultaram em indagações no decorrer desse trabalho e devem ser fruto de um novo estudo para investigar detalhadamente a técnica para simulação do aumento de carga no sistema.

Recomenda-se, também, o emprego do mesmo estudo em sistemas elétricos de grande porte. 


\section{Referências bibliográficas}

[1] PRADA, R. B.; PALOMINO, E. G. C.; DOS SANTOS, J. O. R.; BIANCO, A. ; PILOTTO, L. A. S.. Voltage stability assessment for real-time operation. Generation, Transmission and Distribution, IEE Proceedings, 149:175 - 181, 042002.

[2] KUNDUR, P.. Power system stability and control. McGraw-Hill Professional Publishing, 1994.

[3] ZAMBRONI DE SOUZA, A.; W. MOHN, F.; F. BORGES, I. ; OCARIZ, T.. Using pv and qv curves with the meaning of static contingency screening and planning. Lancet, 81:1491-1498, 072011.

[4] ELGERD, O. I.. Electric energy systems theory: an introduction. McGraw-Hill Book Company, New York, NY, New York, 1982.

[5] VIEIRA FILHO, X.. Operação de sistema de potência com controle automático de geração. Campus, 1984.

[6] MONTICELLI, A. J.. Fluxo de carga em redes de energia elétrica. Editora Blucher, São Paulo, 1983.

[7] BORGES, C. L. T.. Apostila do curso de análise de sistemas de potência. Departamento de Engenharia Elétrica - UFRJ, 2005.

[8] PRADA, R. B.. Notas da disciplina de estabilidade de tensão do mestrado da puc - rio. Departamento de Engenharia Elétrica - PUC RIO, 2017.

[9] CHOWDHURY, B. H.; TAYLOR, C. W.. Voltage stability analysis: $\mathrm{Vq}$ power flow simulation versus dynamic simulation. IEEE Transactions on Power Systems, 15(4):1354-1359, 2000.

[10] GAO, B.; MORISON, G. ; KUNDUR, P.. Voltage stability evaluation using modal analysis. IEEE Transactions on Power Systems, 7(4):1529-1542, 1992.

[11] POWERWORLD CORPORATION. Steady-state power system security analysis with powerworld simulator, s7: Voltage 
stability using qv curves. https://www.powerworld.com/files/ S07QVCurves.pdf, 2008. [Online; Acesso em 10 Jun. 2019].

[12] TAYLOR, C. W.. Power system voltage stability. MCGraw-hill, 1994.

[13] VAN CUTSEM, T.; VOURNAS, C.. Voltage stability of electric power systems. Springer Science \& Business Media, 2007.

[14] CEPEL. Programa de Análise de Redes-ANAREDE V10-02-03-Manual do Usuário. Relatório Técnico CEPEL, No. DP-DSE, 2017.

[15] PRADA, R. B.; SANTOS, J. O. D. ; POMA, C. E. P.. Avaliação das condições de estabilidade de tensão na supervisão e controle de sistemas elétricos em tempo real. Departamento de Engenharia Elétrica - PUC RIO e Cepel, 2012.

[16] UNIVERSITY OF WASHINGTON. Power system test case archive. ieee 14 bus. https://labs.ece.uw.edu/pstca/pf14/pg_tca14bus. htm. [Online; Acesso em 17 Jun. 2019].

[17] AJJARAPU, V.; CHRISTY, C.. The continuation power flow: a tool for steady state voltage stability analysis. IEEE Transactions on Power Systems, 7(1):416-423, 1992. 


\section{A}

\section{Dados de Sistema IEEE 14 Barras}

Nesse apêndice são apresentados os dados utilizados como referência para as simulações no Matlab e no ANAREDE para estudos do sistema IEEE 14 Barras. Os dados de rede são encontrados em [54] e seu diagrama unifilar do sistema é ilustrado na Figura A.1.

(G) Geradores

(C) Compensadores

Síncronos

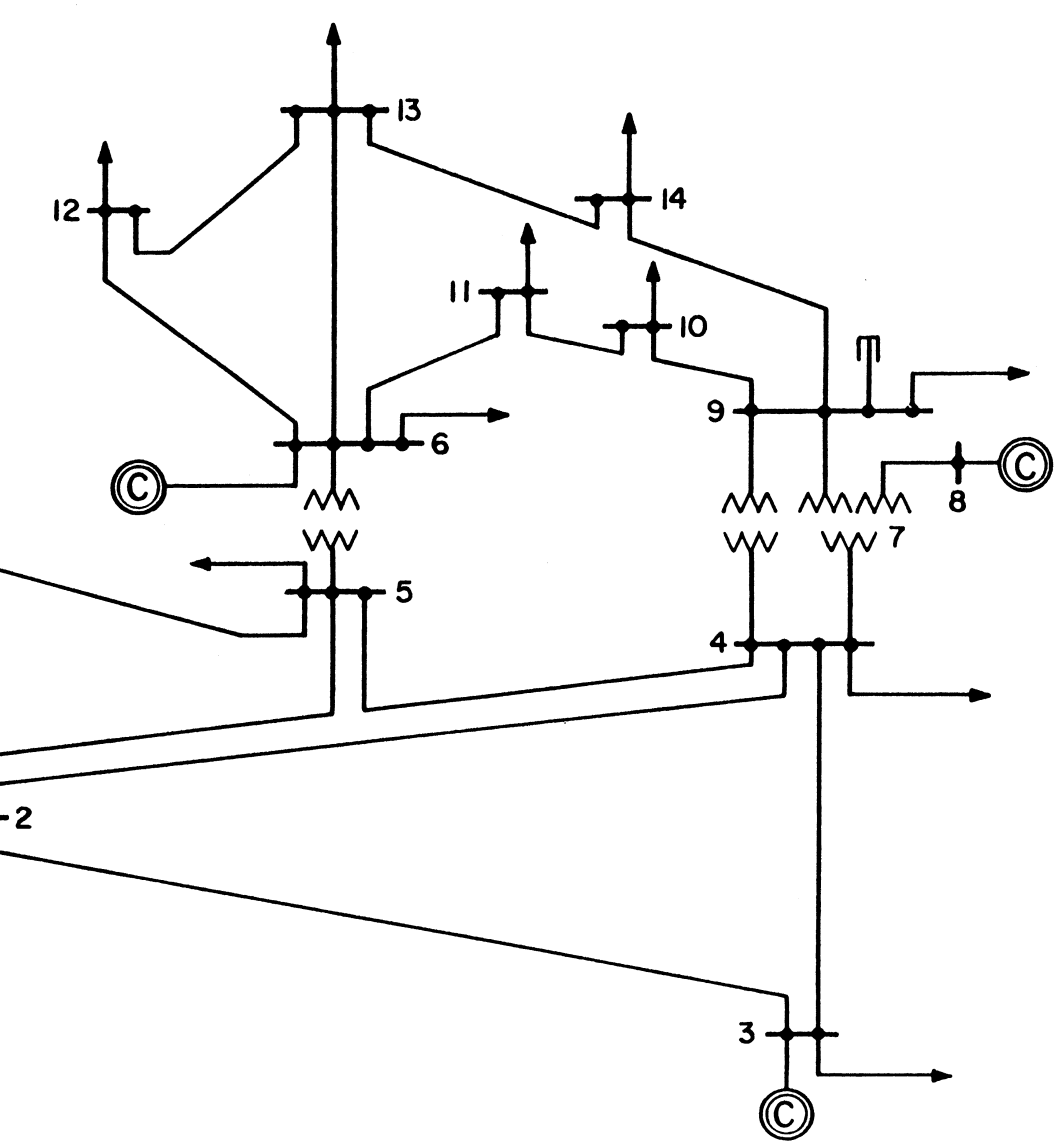

Figura A.1: Diagrama Unifilar do Sistema IEEE 14 Barras[16]

Os dados da rede também podem ser visualizados no formato de entrada para o ANAREDE no Arquivo A. 
Arquivo A.1: Dados de configuração Anarede do Sistema IEEE 14 Barras [15]

TITU

IEEE 14 Bus Test Case - Winter 1962

DOPC IMPR

(Op) E (Op) E (Op) E (Op) E (Op) E (Op) E (Op) E (Op) E (Op) E (Op) E VLIM L CREM L CTAP L QLIM L NEWT L RCVG L FILE L 80CO L RBAR L BPSI L IMPR L

99999

DBAR

(Num)OETGb( nome ) Gl( V) ( A ) ( Pg)( Qg)( Qn)( Qm) (Bc )( Pl)( Ql)( Sh)Are(Vf) 12 Barra-01--HV 1060 0.0232.3-16.6-999999999 1

21 Barra-02--HV 1045-5. 40. 43.37-40. 50. $221.7 \quad 12.7 \quad 11000$

31 Barra-03--HV 1010-13. 0. 25.03 0. 40. $394.219 . \quad 11000$

40 Barra-04--HV 1019-10.

$47.8-3.9 \quad 11000$

5 O Barra-05--HV 1020-8.8

$\begin{array}{lll}7.6 & 1.6 & 11000\end{array}$

61 Barra-06--LV 1070-14. 0.12 .65 -6. 24. $\quad 611.27 .5 \quad 11000$

7 0 Barra-07--ZV 1062-13. 11000

81 Barra-08--TV 1090-13. 0.17 .58 -6. 24. $8 \quad 11000$

90 Barra-09--LV 1056-15.

10 O Barra-10--LV 1051-15.

29.516 .6 19. 11000

110 Barra-11--LV 1057-14.

9. $5.8 \quad 11000$

120 Barra-12--LV 1055-15.

$3.5 \quad 1.8 \quad 11000$

130 Barra-13--LV 1050-15.

$\begin{array}{lll}6.1 & 1.6 & 11000\end{array}$

140 Barra-14--LV 1036-16.

$13.5 \quad 5.8$

11000

99999

14.95 .

11000

DLIN

(De ) d O d ( Pa ) NcEP ( R\% ) ( X\% ) (Mvar) (Tap) (Tmn) (Tmx) (Phs) (Bc ) (Cn) (Ce) Ns

21

1.9385 .917

5.28

51

5.40322 .304

4.92

31

4.69919 .797

4.38

41

5.81117 .632

3.40

51

5.69517 .388

3.46

41

6.70117 .103

1.28

51

$1.335 \quad 4.211$

71

0.00020 .912

0.978

91

0.00055 .618

0.969

61

0.00025 .202

0.932

$\begin{array}{lll}11 & 1 & 9.49819 .890\end{array}$

$\begin{array}{lll}12 & 1 & 12.29125 .581\end{array}$

$\begin{array}{lll}13 & 1 & 6.61513 .027\end{array}$

$\begin{array}{lll}8 & 1 & 0.00017 .615\end{array}$

$\begin{array}{lll}9 & 1 & 0.00011 .001\end{array}$

$\begin{array}{lll}10 & 1 & 3.1818 .450\end{array}$

$9 \quad 10$

$\begin{array}{lll}14 & 1 & 12.71127 .038\end{array}$

8.20519.207

22.09219 .988 